\title{
Mitochondrial metabolism in hypoglossal motoneurons from mouse - implications for amyotrophic lateral sclerosis (ALS)
}

\author{
Ph.D. Thesis \\ In partial fulfilment of the requirements for the degree of Doctor of Philosophy (Ph.D.) \\ in the graduate program Neurosciences at the Georg-August-Universität Göttingen, \\ Faculty of Biology
}

Submitted by

Friederike Bergmann

born in

Wuppertal, Germany

Göttingen 2003 
Advisor, first member of FAC: Prof. Dr. Bernhard U. Keller Second member of FAC: Prof. Dr. Walter Paulus Third member of FAC: Prof. Dr. Erwin Neher

Date of submission of the Ph.D. thesis: 10 December 2003

Day of thesis defense (disputation): 12 February 2004 


\section{CONTENTS}

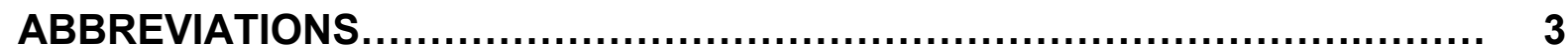

1. INTRODUCTION................................................................ 4

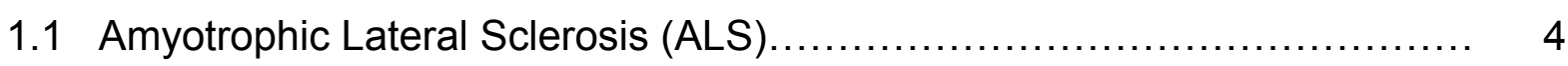

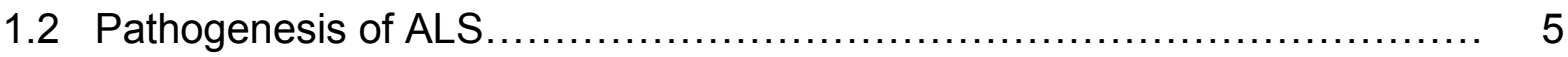

1.3 Disruption of intracellular $\mathrm{Ca}^{2+}$ homeostasis in ALS ......................... 5

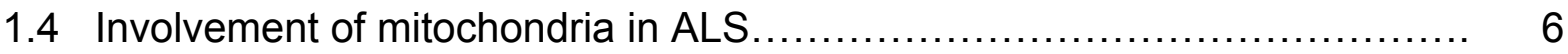

1.5 Factors underlying selective vulnerability of motoneurons in ALS ............. 7

1.6 Mitochondrial function in cell physiology................................. 8

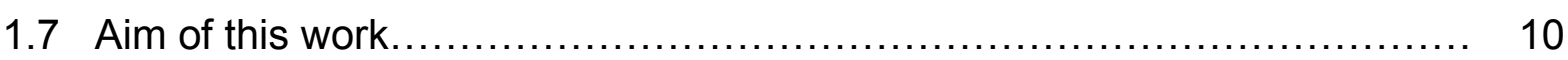

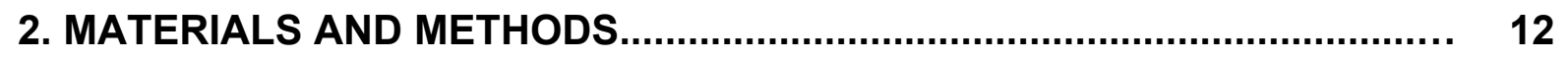

2.1 Preparation of acute mouse brainstem slices.................................... 12

2.2 Experimental set-up............................................................... 13

2.3 Recording of rhythmic motor activity......................................... 14

2.4 Identification of hypoglossal motoneurons in the slice........................... 15

2.5 Electrophysiological recordings.................................................... 15

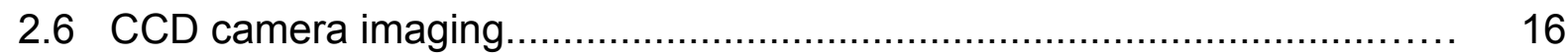

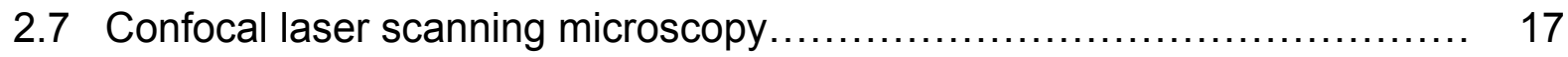

2.8 Microfluorometric calcium measurements......................................... 17

2.9 Analysis of calcium dynamics - the linear compartment model.............. 19

2.10 Estimation $\mathrm{Ca}^{2+}$ clearance rates using polynomial fits.................... 19

2.11 Monitoring of mitochondrial parameters............................... 20

2.12 Multiphoton imaging of intrinsic NADH fluorescence..................... 20

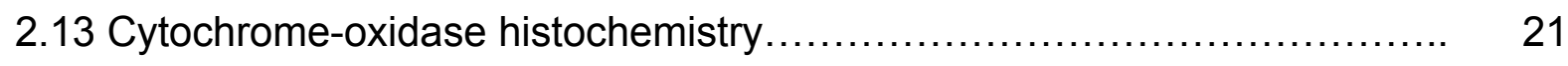

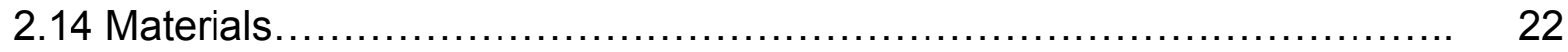

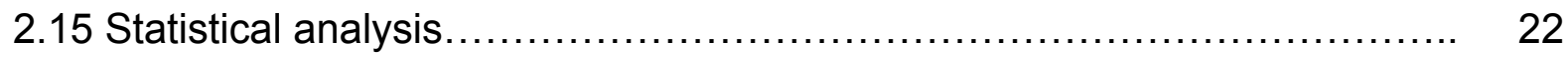


3.1 Mitochondrial $\mathrm{Ca}^{2+}$ buffering in mouse hypoglossal motoneurons...... 23

3.1.1 Stimulation of $\mathrm{Ca}^{2+}$ transients in motoneurons loaded with fura-2/AM.. 23

3.1.2 Contribution of mitochondria to $\mathrm{Ca}^{2+}$ clearance......................... 25

3.1.3 Temperature dependence of mitochondrial $\mathrm{Ca}^{2+}$ buffering............. 28

3.1.4 Impact of mitochondrial $\mathrm{Ca}^{2+}$ buffering on volt.-act. $\mathrm{Ca}^{2+}$ currents.... 29

3.1.5 Impact of dye affinity ............................................. 31

3.1.6 Quantification of mitochondrial $\mathrm{Ca}^{2+}$ clearance rate................... 32

3.1.7 Evaluation of mitochondrial activity and distribution.................... 34

3.2 Impact of mitochondrial dysfunction (CN) on motoneuron membrane

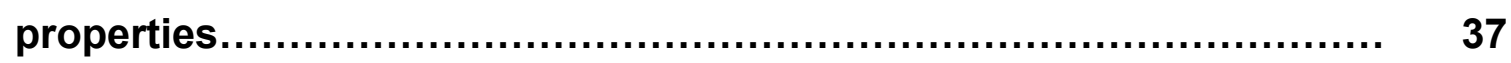

3.2.1 Impact of $\mathrm{CN}$ on rhythmic motor activity ............................. 37

3.2.2 Impact of $\mathrm{CN}$ on electrical properties of hypoglossal MNs............. 38

3.2.3 Differential response of vulnerable and resistant neurons to $\mathrm{CN}$...... 40

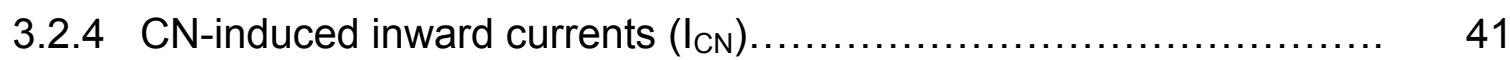

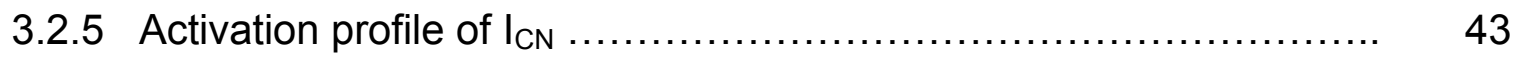

3.3 Impact of mitochondrial dysfunction (CN) on motoneuron $\mathrm{Ca}^{2+}$ levels.. $\quad 48$

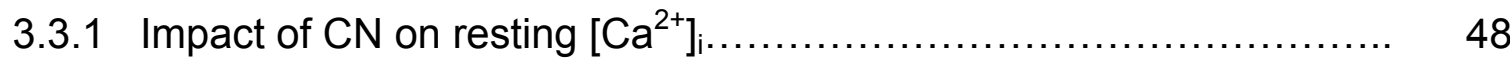

3.3.2 $\mathrm{CN}$ releases $\mathrm{Ca}^{2+}$ from mitochondria-controlled store.................. 50

3.3.3 Impact of $\mathrm{CN}$ on activity-dependent $\mathrm{Ca}^{2+}$ elevations................... 53

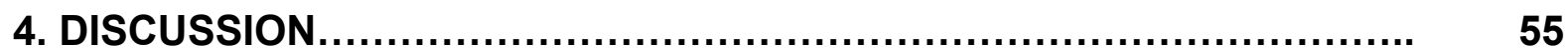

4.1 Dominant role of mitochondria in clearance of physiological $\mathrm{Ca}^{2+}$ loads in hypoglossal motoneurons ............................................. $\quad 55$

4.2 Mechanisms underlying efficient mitochondrial $\mathrm{Ca}^{2+}$ uptake................ 56

4.3 Consequences of disturbed mitochondrial metabolism..................... 58

4.4 Selective vulnerability of motoneurons ..................................... 60

4.5 Implications for the pathogenesis of ALS ................................. 61

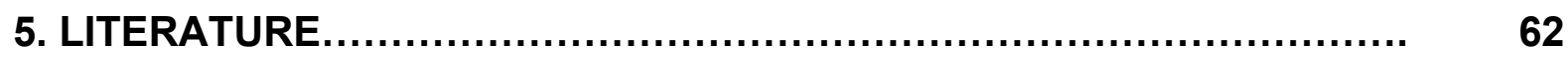

6. CURRICULUM VITAE............................................................ 69 


\section{SUMMARY}

Motoneurons (MNs) are selectively damaged both in human amyotrophic lateral sclerosis (ALS) and corresponding mouse models of this neurodegenerative disease. A variety of studies indicate that mitochondrial dysfunction and disruption of the cellular $\mathrm{Ca}^{2+}$ homeostasis represent critical events during the disease process. Since little is known about the involvement of mitochondria in regulation of $\mathrm{Ca}^{2+}$ levels in MNs, the first aim of this work was to define the contribution of mitochondria to the clearance of physiological type $\mathrm{Ca}^{2+}$ loads. Second, the work aimed at characterizing the cellular consequences of mitochondrial dysfunction in MNs, with particular attention to changes in electrical properties and alterations in $\mathrm{Ca}^{2+}$ homeostasis, as this may give clues to the understanding of processes involved in $\mathrm{MN}$ degeneration and the selective vulnerability of MNs in ALS.

The contribution of mitochondria to buffering of $\mathrm{Ca}^{2+}$ loads was investigated employing acute mouse brainstem slices containing the hypoglossal motor nucleus and $C C D$ camera based imaging techniques. It was demonstrated that in hypoglossal MNs, mitochondria constitute the dominant $\mathrm{Ca}^{2+}$ clearance mechanism accounting for buffering of $\sim 50 \%$ of voltage activated $\mathrm{Ca}^{2+}$ loads with amplitudes below $0.4 \mu \mathrm{M}$. The mitochondrial clearance rate constant $\left(\gamma_{\text {mito }}\right)$ was approximated as $70 \mathrm{~s}^{-1}$. By varying bath temperatures from 19 to $32{ }^{\circ} \mathrm{C}$, mitochondrial $\mathrm{Ca}^{2+}$ buffering was shown to be strongly temperature dependent $(\mathrm{Q} 10=2.1)$. Complementary experiments indicated that the high efficiency of mitochondrial $\mathrm{Ca}^{2+}$ buffering even for low amplitude $\mathrm{Ca}^{2+}$ elevations is sponsored by a high level of mitochondrial activity and a significant interaction of mitochondria and areas of $\mathrm{Ca}^{2+}$ accumulation around influx sites.

To elucidate the consequences of mitochondrial dysfunction on MN membrane properties and intracellular $\mathrm{Ca}^{2+}$ homeostasis, hypoglossal MNs were patch-clamped within the brain slice and mitochondrial function was disturbed using the complex IV inhibitor sodium cyanide (CN). CN activated a TTX-insensitive $\mathrm{Na}^{+}$conductance, which depolarised MNs by $10.2 \pm 1.1 \mathrm{mV}$ and consequently increased spontaneous action potential activity. Reactive oxygen species (ROS) originating at the respiratory chain were identified as potential mediators of the $\mathrm{Na}^{+}$current. $\mathrm{CN}$ responses in 
hypoglossal MNs were further characterized by a significant increase in the cytosolic $\mathrm{Ca}^{2+}$ load following four different mechanisms: i) an increased $\mathrm{Ca}^{2+}$ influx during elevated firing rates, ii) $\mathrm{Ca}^{2+}$ release from mitochondrial stores, iii) retardation of cytosolic $\mathrm{Ca}^{2+}$ clearance rates due to lacking mitochondrial $\mathrm{Ca}^{2+}$ buffering and (iv) a drop in cellular ATP levels. Taken together, the observations identify mitochondrial dysfunction as a critical stressor of motoneuron function, potentially contributing to MN degeneration in ALS. The findings further support a model, where the selective vulnerability of MNs results from a synergistic accumulation of risk factors, including low cytosolic $\mathrm{Ca}^{2+}$ buffering, strong mitochondrial control of $\left[\mathrm{Ca}^{2+}\right]_{\text {, }}$, and a weak protection against increases in electrical excitability during metabolic disturbances. 
ABBREVIATIONS

\begin{tabular}{ll} 
aCSF & artificial cerebrospinal fluid \\
ALS & amyotrophic lateral sclerosis \\
AP & action potential \\
AP-5 & D(-)-2-amino-5-phosphonopentanoic acid \\
ATP & adenosin triphosphate \\
CC & current clamp \\
CCCP & carbonyl cyanide 3-chlorophenylhydrazone \\
CN & cyanide \\
CPA & cyclopiazonic acid \\
CNQX & 6-cyano-7-nitroquinoxaline-2,3-dione \\
FCCP & carbonyl cyanide 4-trifluoro-methoxyphenylhydrazone \\
HP & holding potential \\
MN & motoneuron \\
NADH & nicotinamide adenine dinucleotid , reduced form \\
Rhod123 & rhodamine 123 \\
ROI & region of interest \\
ROS & reactive oxygen species \\
SOD1 & Cu/Zn super oxide dismutase \\
TEA & tetraethylammonium chloride \\
TTX & tetrodotoxin \\
VC & voltage clamp \\
$\Delta \psi$ & mitochondrial membrane potential \\
\hline
\end{tabular}




\section{INTRODUCTION}

\subsection{Amyotrophic Lateral Sclerosis (ALS)}

Amyotrophic lateral sclerosis (ALS) is a neurodegenerative disease clinically characterized by progredient loss of muscle force and breathing capacity, swallowing difficulties as well as spasticity of upper and lower limbs. The incidence is 1-2 per 100.000 persons per year; the age of onset varies from mid 20 to mid 70 with most patients being affected between 53 and 57 years. About $90 \%$ of ALS cases are sporadic, the remaining $10 \%$ are of genetic origin, mainly with an autosomal dominant inheritance. Clinically there is no apparent difference in onset or progression between sporadic and familial forms, which has led researchers to hypothesize that both forms share at least some identical components in their pathogenesis and are very likely to share a common final pathway.

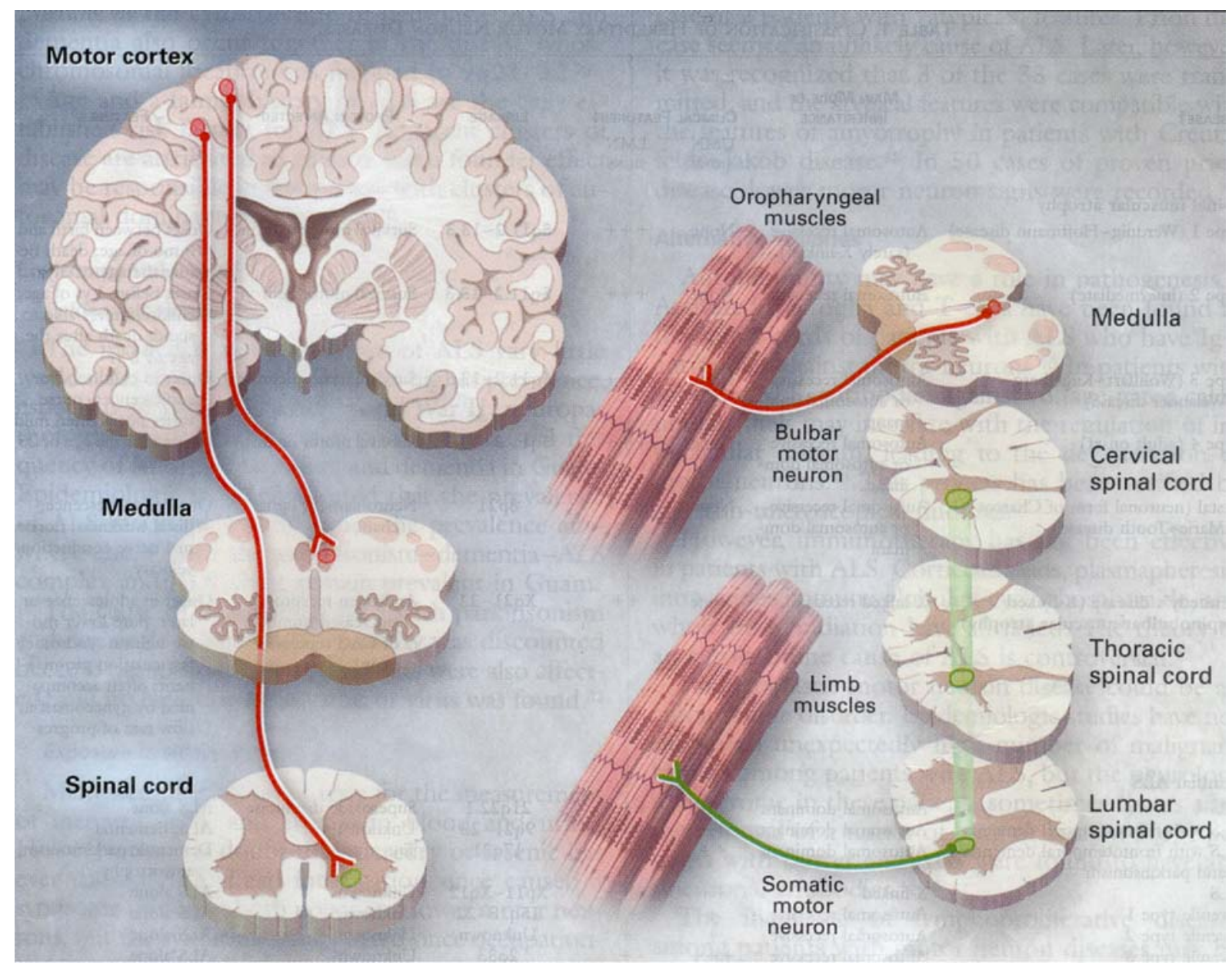

Fig. 1.1: Motoneurons that degenerate in ALS include primary motoneurons in the motor cortex, brainstem motoneurons in motor nuclei and motoneurons in the ventral horn of the spinal cord (taken from Rowland \& Shneider, 2001). 


\subsection{Pathogenesis of ALS}

Corresponding to the clinical picture, ALS is characterized by a progredient loss of cortical, spinal and brain stem motoneurons (Fig. 1). As for most neurodegenerative diseases, the mechanisms leading to selective degeneration of motoneurons in ALS are far from being understood. For sporadic forms of ALS, several pathogenic factors have been proposed, including glutamate excitotoxicity (Rothstein et al., 1992; Heath \& Shaw, 2002), neurofilament disruption (Julien et al., 1998) and excess Ca ${ }^{2+}$ influx through neuronal $\mathrm{Ca}^{2+}$ channels (Appel et al., 1994). As for the familial forms of ALS, $5-10 \%$ are caused by mutations in the gene encoding $\mathrm{Cu} / \mathrm{Zn}$ superoxide dismutase (SOD1).

\section{SOD1-mediated injury}

Mutations in the SOD1 gene underlying a familiar form of ALS were discovered in 1993 (Rosen et al., 1993) and still are the only proven cause for ALS. Generation of transgenic mice expressing human mutant SOD1 (mtSOD1) as a model for ALS (Gurney et al., 1994) has greatly helped researchers to investigate the mechanisms underlying ALS. So far, studies suggest that mtSOD1 affects motoneurons by a "toxic gain of function" rather than a loss of function. This assumption is based on the observations that i) knock-out mice, which completely lack the SOD1-gene, do not develop a phenotype of motoneuron degeneration (Reaume et al., 1996) and ii) 6times over-expression of wildtype SOD1 in mtSOD1-mice does not rescue these mice from motoneuron degeneration (Bruijn et al., 1998). The idea of a "gain of function" is further supported by the fact that more than 90 different ALS-associated mutations in the SOD1 protein have been described with no correlation of enzyme activity and severity of disease (Bowling et al., 1995). However, the exact mechanism by which mtSOD1 affects motoneurons still needs to be clarified.

\subsection{Disruption of intracellular $\mathrm{Ca}^{2+}$ homeostasis in ALS}

Independent of the cellular/molecular event initiating motoneuron degeneration in ALS, disruption of intracellular $\mathrm{Ca}^{2+}$ homeostasis is thought to play a key role in the disease process. Elevated $\mathrm{Ca}^{2+}$ levels have been found in motor nerve terminals from ALS patients (Siklos et al., 1996), in motoneurons of mice after transferring antibodies from ALS patients (Engelhardt et al., 1995) and in motoneurons from mtSOD1 transgenic mice (Siklos et al., 1998). The crucial role of $\mathrm{Ca}^{2+}$ was 
furthermore demonstrated in cell culture models of ALS, where motoneuron degeneration was either induced by AMPA/kainite exposure (Carriedo et al., 1996) or by transfection of mtSOD1 (Roy et al., 1998). Finally, a disruption of intracellular $\mathrm{Ca}^{2+}$ homeostasis as central event in the disease process is supported by experiments where an increase in cytosolic $\mathrm{Ca}^{2+}$ buffers such as parvalbumin protected motoneurons from degeneration both in cell culture and in the mtSOD1 mouse model of ALS (Ho et al., 1996; Beers et al., 2001; Van Den Bosch et al., 2002).

\subsection{Involvement of mitochondria in ALS}

Increasing evidence indicates an involvement of mitochondria in ALS, both in sporadic and in mtSOD1-related forms (Menzies et al., 2002b). First, structural alterations of mitochondria, such as swelling, were observed in motoneurons of ALS patients and transgenic SOD1 mice (Sasaki \& Iwata, 1996; Kong \& Xu, 1998; Fig. 1.2).

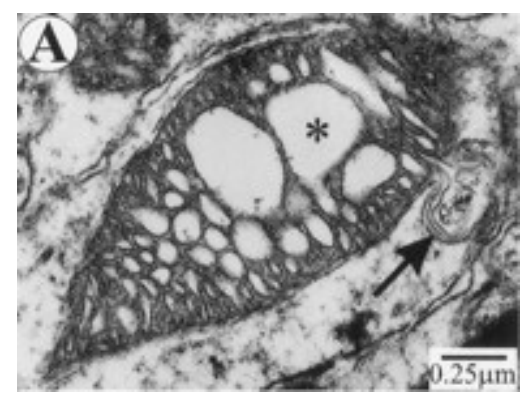

Fig. 1.2: Mitochondria in motoneurons of mutant SOD1 transgenic mice show abnormalities like dilated cristae (asterisk) and leaking outer membrane (arrow; taken from Kong \& Xu, 1998).

More recently, dysfunction of mitochondria has been described in ALS. This dysfunction was partially associated with mutations in mitochondrial DNA and altered mitochondrial electron chain activity (Swerdlow et al., 1998; Borthwick et al., 1999; Wiedemann et al., 2002). In mtSOD1 based ALS, mitochondrial dysfunction has been shown to be induced by the mutated protein itself, which inhibits complex II and IV of the respiratory chain (Menzies et al., 2002a). That mitochondrial dysfunction is causally related to motoneuron degeneration rather than being a secondary event is suggested by the fact that it occurs during early disease stages (Jung et al., 2002). Even more convincing is the observation that chronic mitochondrial inhibition may induce selective motoneuron degeneration in vivo and in vitro (Kaal et al., 2000). 


\subsection{Factors underlying selective vulnerability of motoneurons in ALS}

The fact that certain cell types such as spinal or brainstem motoneurons are primarily affected in ALS, whereas others are usually spared, is called "selective vulnerability". Many studies have been undertaken to elucidate the features determining selective motoneuron vulnerability in ALS. One important factor that has been determined is a low cytosolic $\mathrm{Ca}^{2+}$ buffering capacity ( $\kappa_{S}$ ) corresponding to a low expression of $\mathrm{Ca}^{2+}$ binding proteins (i.e. calbindin, parvalbumin, calretinin) in vulnerable cells (Lips \& Keller, 1998; Palecek et al., 1999; Vanselow \& Keller, 2000; Fig. 1.3). Under physiological conditions, low $\kappa_{S}$ enables fast and highly localized cytosolic $\mathrm{Ca}^{2+}$ signalling at low energy cost, but presumably enhances the vulnerability of motoneurons under pathophysiological conditions. Whereas vulnerable motoneurons show $\kappa_{S}$ values in the range of 40 , cells that are usually not affected in ALS such as oculomotor neurons, hippocampal CA1 or Purkinje neurons display several-fold higher $\kappa_{S}$ values ( 200, 250 and 800 , respectively).
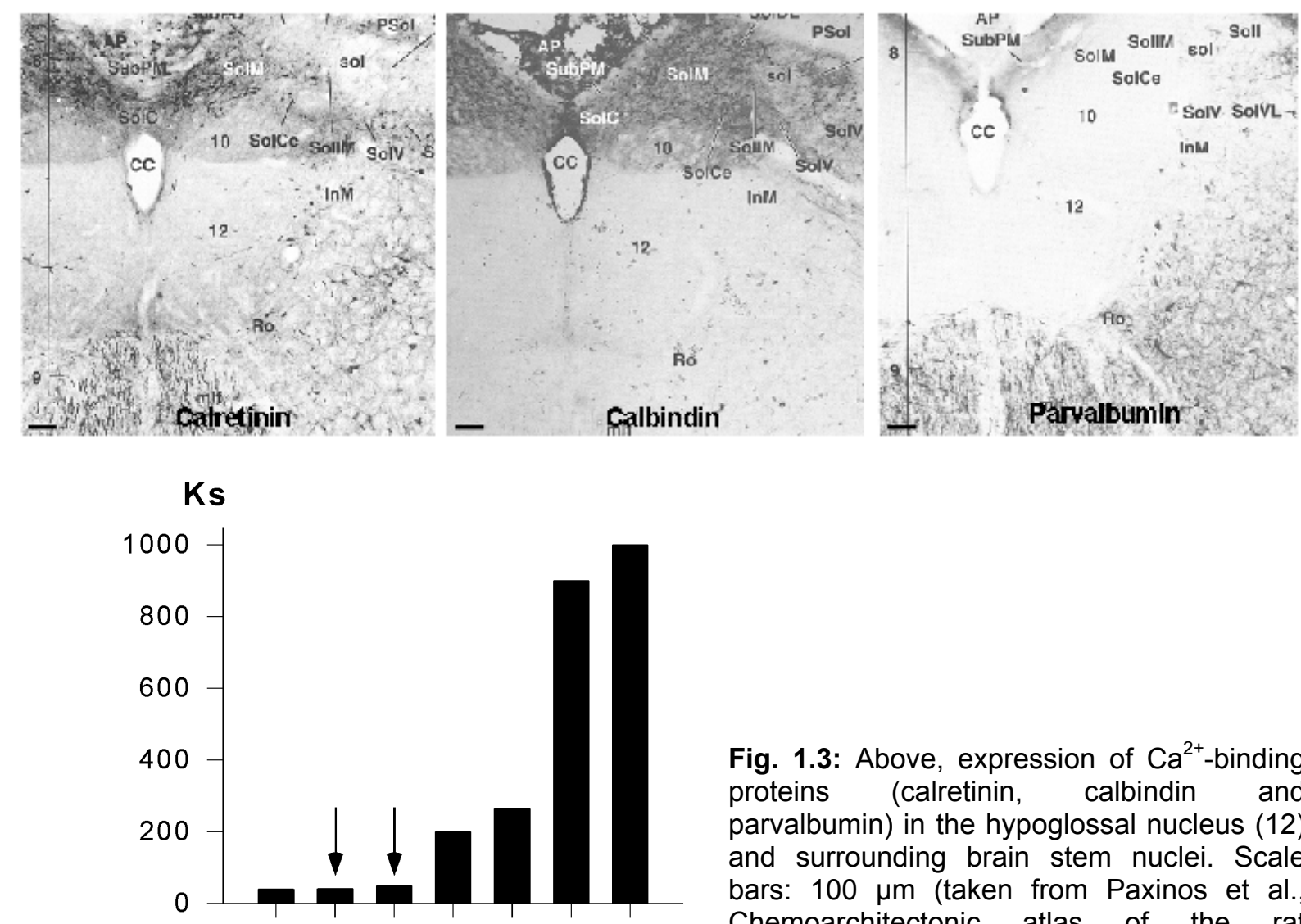

Fig. 1.3: Above, expression of $\mathrm{Ca}^{2+}$-binding proteins (calretinin, calbindin and parvalbumin) in the hypoglossal nucleus (12) and surrounding brain stem nuclei. Scale bars: $100 \mu \mathrm{m}$ (taken from Paxinos et al., Chemoarchitectonic atlas of the rat brainstem, Academic Press, 1999). Below, comparison of $\kappa_{S}$ values between different cell types. Vulnerable motoneurons are marked by arrows. 
Support for the concept that low cytosolic $\mathrm{Ca}^{2+}$ buffering contributes to the selective motoneuron vulnerability is provided by experiments, in which a controlled increase in cytosolic $\mathrm{Ca}^{2+}$ buffers rescued motoneurons from ALS-associated degeneration in cell culture models but also in living mice expressing mtSOD1 (Roy et al., 1998; Beers et al., 2001; Van Den Bosch et al., 2002).

Further factors that are proposed to support a selective motoneuron vulnerability in ALS are the presence of $\mathrm{Ca}^{2+}$ permeable $\alpha$-amino-3-hydroxy-5-methylisoxazole-4propionic acid (AMPA) receptors (Shaw \& Eggett, 2000; Vandenberghe et al., 2000) and a high neurofilament content (Julien et al., 1998). Additionally, a high level of mitochondrial activity serving the high energy requirements of motoneurons is thought to contribute to it (Menzies et al., 2002b). This assumption is supported by the observation that mitochondrial inhibition induces a selective motoneuron degeneration in vitro (Kaal et al., 2000). Taken together, it seems that no single property, but rather an accumulation of a variety of factors is responsible for the vulnerability of motoneurons to ALS-associated damage.

\subsection{Mitochondrial function in cell physiology}

As this work will focus on the role of mitochondria in motoneurons and ALS, the physiological properties of mitochondria will be briefly summarized.

\section{ATP generation}

Mitochondria have long been known to be the primary energy source in mammalian cells. ATP is generated by the $F_{0} F_{1}$ ATP synthase located in the inner mitochondrial membrane, which utilizes the existence of a proton gradient over the inner mitochondrial membrane for ATP synthesis. This proton gradient, which also constitutes a potential gradient ( $\Delta \psi$, mitochondrial matrix more negative than cytosol), is generated by an efflux of protons during electron transport at the respiratory chain (Fig. 1.4). The most important proton donor is nicotinamide adenine dinucleotid (NADH) constituting also the source of electrons for the respiratory chain. When spending proton and electron at the level of complex I, NADH is oxidized to NAD'. Regeneration to NADH takes place in the citrate cycle in the mitochondrial matrix. 


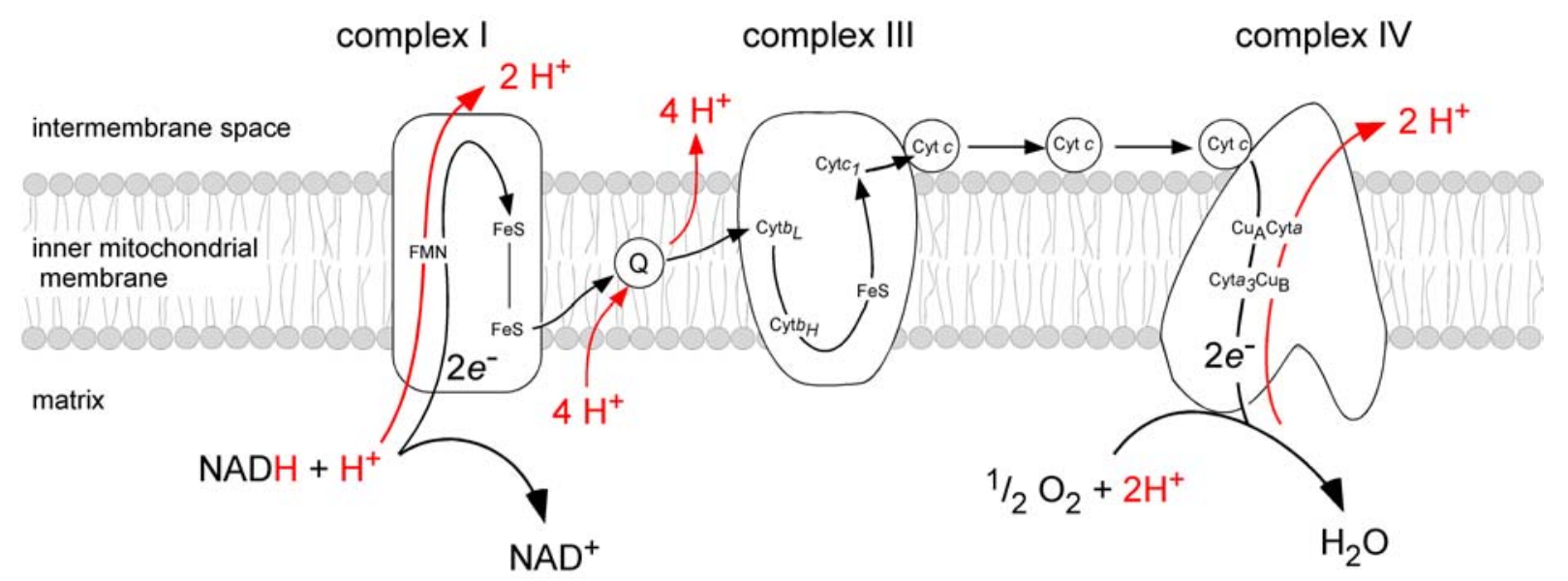

Fig. 1.4: Illustration of the mitochondrial respiratory chain located in the inner mitochondrial membrane. The pathway of electron transport is indicated in black, proton pumping in red. The fluorescent substrate $\mathrm{NADH}$ is oxidized to the non-fluorescent $\mathrm{NAD}^{+}$on the level of complex I (after Voet \& Voet, 1995).

$\underline{\mathrm{Ca}^{2+} \text { cycling }}$

Mitochondria are also known as important players in cellular $\mathrm{Ca}^{2+}$ handling (Duchen, 2000; Nicholls \& Budd, 2000; Rizzuto et al., 2000). This evidence is based on the discovery of active $\mathrm{Ca}^{2+}$ uptake and extrusion mechanisms in the inner membrane of mitochondria. It is generally believed that during a variety of physiological-type stimuli, mitochondria take up $\mathrm{Ca}^{2+}$ via a $\mathrm{Ca}^{2+}$ uniporter driven by the potential gradient $\Delta \psi$. The rate of mitochondrial $\mathrm{Ca}^{2+}$ uptake is believed to depend on the cytosolic $\mathrm{Ca}^{2+}$ concentration, with increasing operation of the uniporter at high cytosolic $\mathrm{Ca}^{2+}$ levels. $\mathrm{Ca}^{2+}$ is mainly brought out of mitochondria via the $\mathrm{Na}^{+} / \mathrm{Ca}^{2+}$ antiporter operating usually at maximum rate. If the rate of mitochondrial $\mathrm{Ca}^{2+}$ uptake exceeds $\mathrm{Ca}^{2+}$ extrusion, then $\mathrm{Ca}^{2+}$ can accumulate in mitochondria (Fig. 1.5). Studies on isolated mitochondria have shown that this occurs when cytosolic $\left[\mathrm{Ca}^{2+}\right]$ reaches levels higher than $300 \mathrm{nM}$. As $\mathrm{Ca}^{2+}$ and phosphate residues can form insoluble complexes in the inner mitochondrial matrix, mitochondria are capable of storing $\mathrm{Ca}^{2+}$ and doing so to compensate for increasing $\mathrm{Ca}^{2+}$ influx.

$\mathrm{Ca}^{2+}$ cycling in mitochondria is tightly linked to oxidative phosphorylation and energy production (Nicholls \& Budd, 2000). This is based on the fact that certain enzymes of the citrate cycle (i.e. pyruvate dehydrogenase, isocitrate dehydrogenase) are activated by increases in mitochondrial matrix $\left[\mathrm{Ca}^{2+}\right]$. The increased activation of the citrate cycle is reflected in an elevated reduction state of $\mathrm{NADH}$, which increases oxidative phosphorylation and ATP synthesis. 


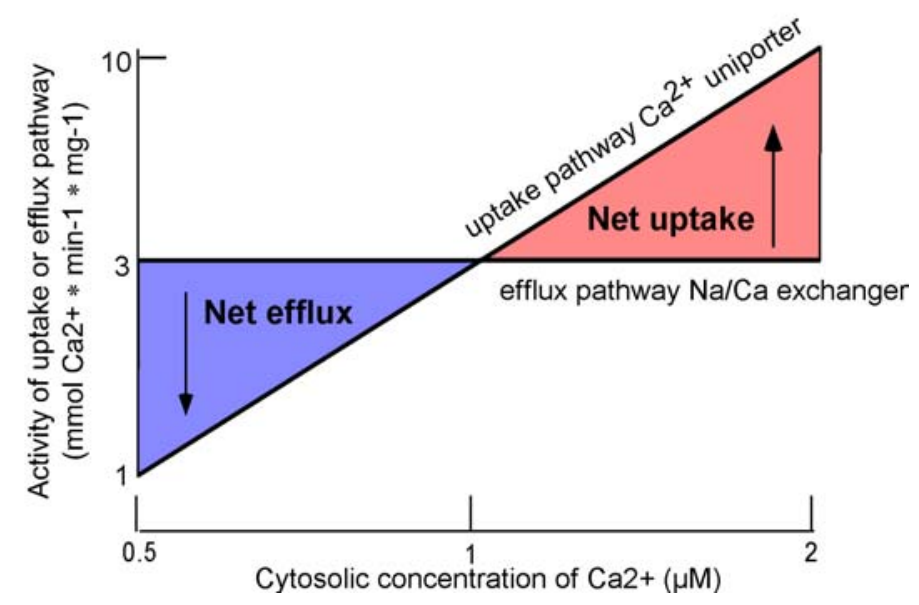

Fig. 1.5: Illustration of the activity of mitochondrial $\mathrm{Ca}^{2+}$ transport systems. Mitochondrial $\mathrm{Ca}^{2+}$ accumulation occurs when the activity of the uptake system ( $\mathrm{Ca}^{2+}$ uniporter) exceeds those of the efflux system $\left(\mathrm{Na}^{+} / \mathrm{Ca}^{2+}\right.$ exchanger; after Voet \& Voet, 1995).

Under certain circumstances, mitochondria can release large amounts of $\mathrm{Ca}^{2+}$ in the cytosol. At least two different pathways for $\mathrm{Ca}^{2+}$ release have been proposed. First, release of $\mathrm{Ca}^{2+}$ by reverse mode action of the $\mathrm{Ca}^{2+}$ uniporter and second, release of $\mathrm{Ca}^{2+}$ via opening of an unspecific cation channel, the mitochondrial permeability transition pore (mPTP). The latter one is usually restricted to pathological conditions.

\section{Free radical production}

Besides important roles in energy production and calcium handling, mitochondria constitute the main source of free radicals in the cell, owing to inefficient transport of electrons in the respiratory chain (Lopez-Barneo et al., 2001). Radicals are produced preferentially at the semiubiquinone site (level of complex III), where an electron can be transferred to $\mathrm{O}_{2}$ to produce superoxide $\left(\mathrm{O}_{2}{ }^{-}\right)$. Superoxide is easily converted to hydrogen peroxide $\left(\mathrm{H}_{2} \mathrm{O}_{2}\right)$ and hydroxyl radicals $(\mathrm{OH})$, altogether being referred to as reactive oxygen species (ROS).

\subsection{Aim of this work}

The present work aims at investigating physiological and pathophysiological aspects of mitochondrial function in motoneurons, considering that disruption of such processes may contribute to motoneuron degeneration in ALS. Of particular interest was the interplay between mitochondrial function and cellular $\mathrm{Ca}^{2+}$ homeostasis as previous studies have identified low endogenous $\mathrm{Ca}^{2+}$ buffering, high amplitude $\mathrm{Ca}^{2+}$ transients and localized $\mathrm{Ca}^{2+}$ profiles as typical features of motoneurons. 
The first aim of this study was therefore to define the impact of mitochondrial function on $\left[\mathrm{Ca}^{2+}\right]_{\text {, }}$, in particular to buffering of $\mathrm{Ca}^{2+}$ transients, which are a characteristic part of motoneuron physiology. The second aim was to evaluate the consequences of a disturbed mitochondrial metabolism, with respect to $\left[\mathrm{Ca}^{2+}\right]_{\mathrm{i}}$ and membrane properties, as this should give clues to understanding of processes involved in motoneuron degeneration and the selective vulnerability of motoneurons in ALS. 


\section{MATERIALS AND METHODS}

\subsection{Preparation of acute mouse brainstem slices}

All electrophysiological and optical recordings described in this work were performed on acute mouse brainstem slices prepared from young (1-5 days old) NMR1 mice. Animal experiments were carried out in accordance with the guidelines of the Ethics Committee of the University of Göttingen. Animals were decapitated and the head was immediately placed into $4^{\circ} \mathrm{C}$ cold artificial cerebrospinal fluid (aCSF, in mM: 118 $\mathrm{NaCl}, 3 \mathrm{KCl}, 1 \mathrm{MgCl}_{2}, 25 \mathrm{NaHCO}_{3}, 1 \mathrm{NaH}_{2} \mathrm{PO}_{4}, 1.5 \mathrm{CaCl}_{2}, 20$ glucose; 320 mosm; $\mathrm{pH} 7.4$ achieved by continuous bubbling with carbogen $\left.\left(95 \% \mathrm{O}_{2}, 5 \% \mathrm{CO}_{2}\right)\right)$. Under visual control, skin and skull cap were removed, permitting free access to the brain. From anterior to posterior, the brain was carefully loosened from the cranial base, the cranial nerves being cut with fine scissors. The isolated brain was then placed in a petri dish filled with aCSF. Using a scalpel, the telencephalon was cut away from cerebellum and brainstem. Subsequently, cerebellum and brainstem were separated by an axial cut. The isolated brainstem was glued on an agar block with the dorsal side facing the agar. The agar block was glued on a $20^{\circ}$ angled block with the rostral side of the brainstem directed upwards. The block was then placed in an aCSFcontaining chamber so that the brainstem's ventral side faced the blade (Fig. 2.1).

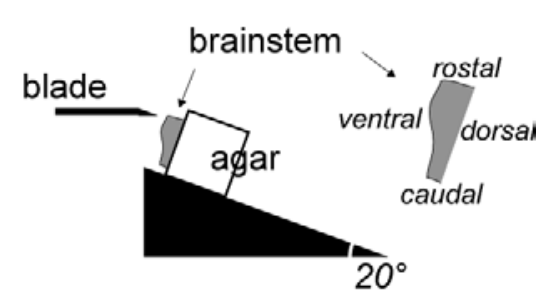

Fig. 2.1: Position of the isolated mouse brainstem during slicing.

Transverse brainstem slices were cut using a vibratome (LeicaVT100S, Leica Instruments $\mathrm{GmbH}$, Nussloch, Germany) from rostral to caudal reaching the region of interest when the $\mathrm{IV}^{\text {th }}$ ventricle had passed its largest diameter. At this level, for recordings of rhythmic motor activity, a single transverse brainstem slice was prepared with a thickness of $\sim 600 \mu \mathrm{m}$ containing the pre-Bötzinger complex and large parts of the hypoglossal nucleus. For all other experiments, transverse slices were cut with a thickness of $\sim 200 \mu \mathrm{m}$. In this way, up to 4 slices could be obtained containing neurons of the hypoglossal nucleus. 


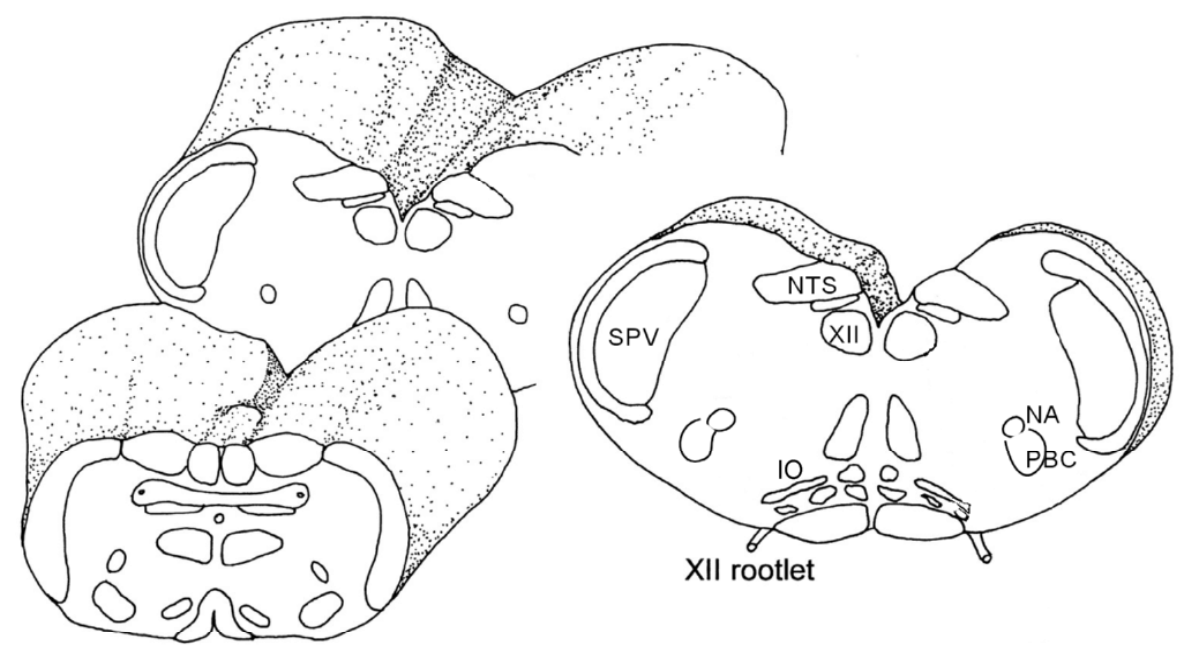

Fig. 2.2: Schematic drawing of the medulla oblongata, from which slices were taken containing the hypoglossal nucleus (XII), ambiguus nucleus (NA), pre-Bötzinger complex (PBC), solitary tract nucleus (NTS), the spinal part of the trigeminal nucleus (SPV) and the inferior olive (IO; modified after Ramirez et al., 1997).

After preparation, $600 \mu \mathrm{m}$ thick slices were directly transferred into the recording chamber and rinsed with $28^{\circ} \mathrm{C}$ warm aCSF. Thin slices $(200 \mu \mathrm{m})$ were transferred to $33{ }^{\circ} \mathrm{C}$ warm aCSF, which then cooled down to room temperature (RT, $20-22^{\circ} \mathrm{C}$ ). In the recording chamber, all slices were fixed with an U-shaped platinum wire, on which nylon strings were glued.

\subsection{Experimental set-up}

In Göttingen, most of the experiments were performed with a set-up allowing simultaneous electrophysiological recording and CCD camera imaging. The recording chamber containing the brain stem slice was fixed on a microscope table under a Zeiss microscope (Axioskope, Zeiss, Göttingen, Germany). The microscope table was movable in an $X Y$ direction under the microscope. Additionally, the microscope was placed on a sliding table (Spindler \& Hoyer, Göttingen, Germany) allowing $X Y$ movements of the microscope over the preparation. The whole set-up was installed on a table providing air-suspension and surrounded by a Faraday cage in order to protect it from vibration and low frequent electric noise. The recording chamber allowed continuous perfusion with aCSF via Tygon ${ }^{\circledR}$ tube connection. For all optical measurements, a 63x water immersion objective (Achroplan, Zeiss) was employed. 


\subsection{Recording of rhythmic motor activity}

Hypoglossal motoneurons (MNs) are part of the respiratory network which generates the rhythm of breathing in mammals. They receive input from the rhythm-generating centre, the pre-Bötzinger complex located in the ventro-lateral medulla, and respiratory-related activity is projected via the hypoglossal nerve (XII) to tongue and pharynx muscles. The function of tongue and pharynx innervation during breathing is to stabilize the upper airway system to prevent its collapsing during inspiration. In 1991, Smith et al. (1991) demonstrated that a $\sim 600 \mu \mathrm{m}$ thick preparation of the lower brainstem, which includes the pre-Bötzinger complex and large parts of the hypoglossal nucleus, can maintain rhythmic respiratory-related activity in vitro. This respiratory-related activity can be recorded as motor output from hypoglossal (XII) rootlets (Fig. 2.3), which was performed in some experiments described in this work.
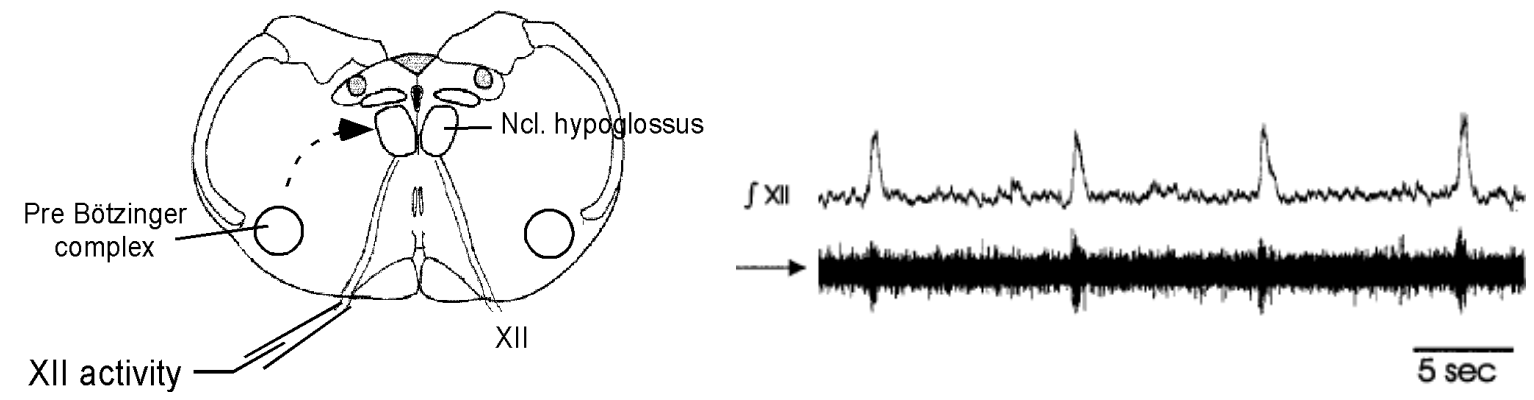

Fig. 2.3: Left, schematic drawing of a brain stem slice containing the respiratory network, where rhythmic activity is generated in the pre-Bötzinger complex and projected to the hypoglossal nucleus. Motor output (XII activity) is recorded from the hypoglossal rootlets. An example of rhythmic motor activity is shown on the right (lower trace). The integrated activity is shown above (modified after Ramirez et al., 1997).

Rhythmic respiratory-related activity of hypoglossal rootlets was recorded using suction electrodes pulled from borosilicate glass tubes (Kimax 51, Kimble Products, Vineland, NY, U.S.A.) and standard electrophysiological equipment (Lips \& Keller, 1998). To obtain regular rhythmic activity, the potassium concentration of the aCSF was raised to $8 \mathrm{mM}$. 


\subsection{Identification of hypoglossal motoneurons in the slice}

In brainstem slices, hypoglossal motoneurons (MNs) were identified by their localization in the hypoglossal nucleus paramedian ventral of the $\mathrm{IV}^{\text {th }}$ ventricle or the central channel (Fig. 2.4). They could furthermore be distinguished from neighbouring neurons by their large size (somatic diameter usually $>15 \mu \mathrm{m}$ ) and their rounded shape. In patch-clamp experiments, suitable MNs were additionally selected by their ability to fire action potentials in a drug-free solution and the occurrence of spontaneous synaptic activity.
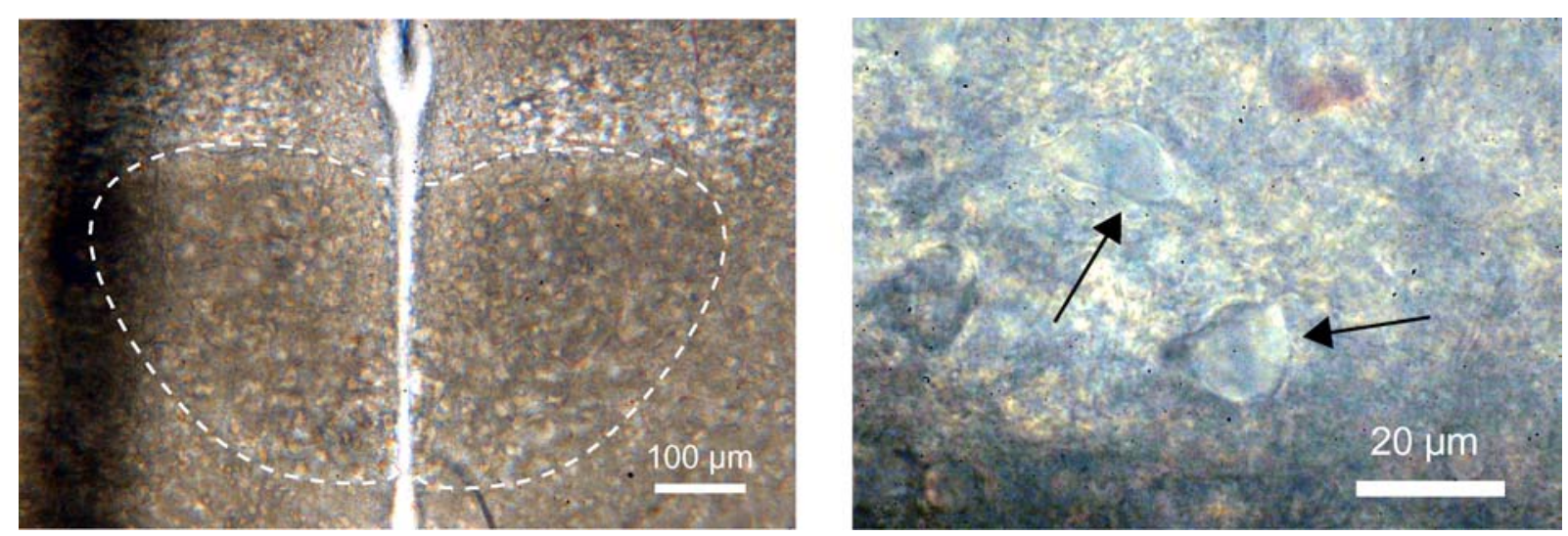

Fig. 2.4: Acute mouse brainstem slices (200 $\mu$ m thick) seen in transmitted light in 10x (left) and 60x (right) magnification. The dashed line surrounds the hypoglossal nucleus paramedian, ventral of the central channel (left); the arrows point to single hypoglossal motoneurons (right).

\subsection{Electrophysiological recordings}

For patch-clamp experiments, patch pipettes were pulled from borosilicate glass tubes (Kimax 51, Kimble Products, Vineland, NY, U.S.A.). The intracellular pipette solution contained $(\mathrm{mM}) 140 \mathrm{KCl}$ (alternatively $120 \mathrm{CsCl}$ and $20 \mathrm{TEA}), 10 \mathrm{HEPES}, 2$ $\mathrm{MgCl}_{2}, 4 \mathrm{Na}_{2} \mathrm{ATP}, 0.4 \mathrm{NaGTP}$ (adjusted to $\mathrm{pH} 7.3$ with $\mathrm{KOH}$ or $\mathrm{CsOH}$ ). When filled with intracellular solution, pipettes displayed resistances of 1.8-3.5 $\mathrm{M} \Omega$. Under visual control, patch pipettes were approached to hypoglossal MNs using 3-dimensional piezo-electric control (Physik Instrumente, Waldbronn, Germany) and continuous application of positive pressure to avoid contamination of the pipette tip and to remove extracellular matrix surrounding hypoglossal MNs. Correct positioning of the pipette tip in close proximity of hypoglossal MNs was indicated by occurrence of a slight membrane cavity. Subsequently, positive pressure was removed and MN membrane was gently sucked into the pipette tip. Membrane seals developed with resistances $>1 \mathrm{G} \Omega$ as indicated by continuous application of test pulses $(-10 \mathrm{mV}$, 
$5 \mathrm{~ms}$ ). The whole-cell configuration (Hamill et al., 1981) was established by rupture of the membrane in the pipette tip. Whole cell recordings in voltage clamp (VC) and current clamp (CC) mode were performed using an EPC-9 patch clamp amplifier (HEKA Electronics, Lambrecht, Germany). After rupture of the seal, series resistance $\left(R_{s}, 6-15 \mathrm{M} \Omega\right.$ ) was continuously monitored. $V C$ recordings were performed only when $R_{s}$ stabilized and cells displaying $R_{s}$ higher than $20 \mathrm{M} \Omega$ were excluded from analysis. In part of the experiments, $R_{S}$ was compensated up to $50 \%$. Voltage and current pulse generation and data acquisition were performed with a Macintosh computer running PULSE software 8.11 (HEKA). Whole-cell currents were recorded with sampling frequencies of 4-10 kHz and filtered (Bessel filter, $2.9 \mathrm{kHz}$ ) before analysis. For analysis, PulseFit (HEKA) and IgorPro (Wavemetrics Inc., Lake Oswego, OR, U.S.A.) software were employed.

\subsection{CCD camera imaging}

CCD camera imaging was applied to monitor dynamic changes in $\left[\mathrm{Ca}^{2+}\right]_{i}$ and mitochondrial parameters of hypoglossal MNs in the slice. Fluorescence excitation was done by using a computer-controlled monochromator (Polychrome II, TILL Photonics, Gräfelfing, Germany), which was connected to an upright microscope via quartz fibre optics and a minimum number of optical components for maximum fluorescence excitation (objective Achroplan W 63x, 0.9W). By this system, wavelengths in the range of 300-700 nm could be generated with a bandwidth of 13 $\mathrm{nm}$, making the use of excitation filters unnecessary. The emitted light was directed to a dichroic mirror with mid reflection depending on the fluorescent dye used (fura: $425 \mathrm{~nm}$, rhod123: $510 \mathrm{~nm}$; Zeiss, Germany). Fluorescence signals were detected with a peltier-cooled $\left(-15^{\circ} \mathrm{C}\right)$ slow scan CCD camera (IMAGO, TILL Photonics) displaying 12-bit dynamics and an $\mathrm{A} / \mathrm{D}$ converter with $12.5 \mathrm{MHz}$ sampling rate. Fluorescence changes in defined "regions of interest" (ROIs) were monitored online using a PC running TILL Vision Software V4.0 (TILL Photonics). To maximize the speed of fluorescence recordings, camera binning was set to $4 \times 4$ (120×160 pixel) allowing sampling rates up to $15 \mathrm{~Hz}$ at reasonable spatial resolution. The analysis of fluorescence signals was performed off-line with IGOR (Wavemetrics, Lake Oswego, OR, U.S.A.) software. 


\subsection{Confocal laser scanning microscopy}

A confocal laser scan system (model 510, Zeiss, Germany) was used for localisation studies of mitochondrial networks. In these experiments, acute slices were loaded with $1 \mu \mathrm{M}$ mitotracker green/AM for $30 \mathrm{~min}$ followed by a 30 minutes wash. Slices were placed into a custom build recording chamber mounted on the microscope table and perfused with aCSF. Mitotracker green was excited using the $488 \mathrm{~nm}$ line of an argon laser and fluorescence was collected using a 505-530 nm band-pass filter. Data acquisition and analysis was done using the LSM software provided by Zeiss.

\subsection{Microfluorometric calcium measurements}

Changes in cytosolic $\left[\mathrm{Ca}^{2+}\right]$ were monitored using fura-2 $\left(\mathrm{K}_{\mathrm{d}} \sim 0.2 \mu \mathrm{M}\right)$ or fura-FF $\left(\mathrm{K}_{\mathrm{d}}\right.$ $\sim 5.5 \mu \mathrm{M})$. The fluorescent dyes were brought into the cells either by addition of their potassium salts to the patch pipette solution (100 $\mu \mathrm{M}$, Fig. 2.5), or by loading the slice with their membrane-permeable AM-ester forms $\left(10 \mu \mathrm{M}\right.$ for 60 min at $33^{\circ} \mathrm{C}$, subsequent wash >30 min at RT). In the latter case, the ester conjugate diffuses into the cell, where the ester group is cleaved so that the dye is trapped in the cytosol.

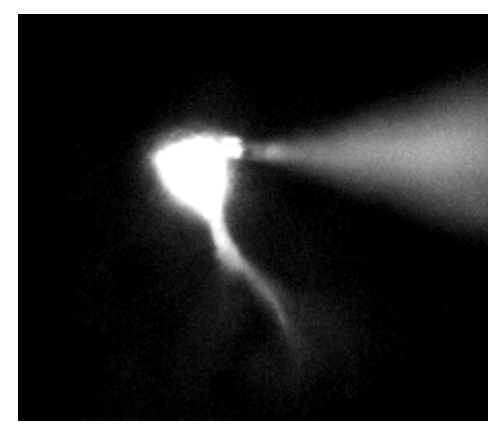

Fig. 2.5: CCD camera image of a patch-clamped hypoglossal motoneuron loaded with $100 \mu \mathrm{M}$ fura-2. Fluorescence excitation was done at $390 \mathrm{~nm}$.

Fura-2 and related $\mathrm{Ca}^{2+}$ indicators are classical ratiometric dyes due to their shift in the excitation spectrum upon binding of $\mathrm{Ca}^{2+}$ (Fig. 2.6). During increase of $\left[\mathrm{Ca}^{2+}\right]_{\mathrm{i}}$, the fluorescence activity of fura decreases when excited at $\sim 385 \mathrm{~nm}$, whereas the fluorescence activity increases when excited at $\sim 340 \mathrm{~nm}$. At $\sim 360 \mathrm{~nm}$, fura displays its isosbestic point, where the emission is not dependent on $\mathrm{Ca}^{2+}$. In the experiments described here, fura was alternately excited at 360 and $385 \mathrm{~nm}$, as the objectives used did not transmit light at wavelengths around $340 \mathrm{~nm}$. Excitation at $360 \mathrm{~nm}$ is furthermore advantageous, because it allows estimation of the dye concentration in the cell (Neher, 1995). 


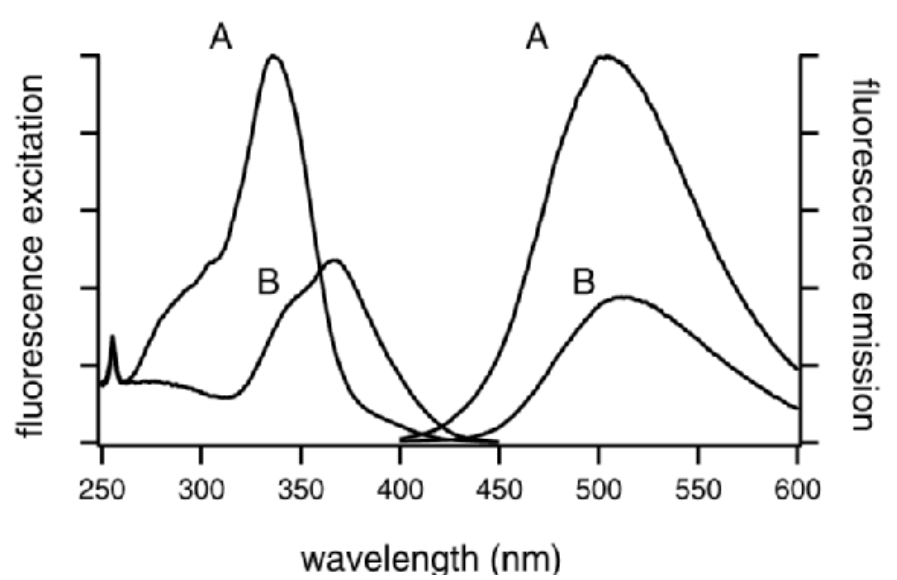

Fig. 2.6: Fluorescence excitation (detected at $510 \mathrm{~nm}$ ) and emission (excited at $340 \mathrm{~nm}$ ) spectra of $\mathrm{Ca}^{2+}$-saturated (A) and $\mathrm{Ca}^{2+}$-free (B) fura-2 in pH 7.2 buffer (taken from Molecular Probes homepage).

For most recordings, where fura was loaded via the patch-pipette, the fluorescence ratios were converted into $\mathrm{Ca}^{2+}$ concentrations after a background correction had been performed. The two main sources of background fluorescence resulted from (1) dye that had distributed in the slice prior to the seal formation and (2) tissue autofluorescence (i.e. NADH, FADH). The background fluorescence was recorded in a ROI drawn beside the patch-clamped cell and to correct for it, the average fluorescence of the background window was subtracted from each image before ratioing. The resulting "background-corrected" ratio trace was transformed into $\left[\mathrm{Ca}^{2+}\right]_{\mathrm{i}}$ using the equation:

$$
\left[\mathrm{Ca}^{2+}\right]_{i}=\mathrm{K}_{\mathrm{d}}{ }^{*}\left(\mathrm{R}_{\max } / \mathrm{R}_{\min }\right){ }^{*}\left(\mathrm{R}-\mathrm{R}_{\min }\right) /\left(\mathrm{R}_{\max }-\mathrm{R}\right) \quad \text { (Grynkiewicz et al., 1985) }
$$

$R_{\max }$ and $R_{\min }$ are the fluorescence ratios for infinite high and infinite low cytosolic $\left[\mathrm{Ca}^{2+}\right] ; \mathrm{K}_{d}$ is the dissociation constant of fura-2. $\mathrm{R}_{\max }$ and $\mathrm{R}_{\min }$ were determined in vivo by loading cells with either $10 \mathrm{mM} \mathrm{Ca}^{2+}\left(\mathrm{R}_{\max }\right)$ or $0 \mathrm{mM} \mathrm{Ca}^{2+}$ and $10 \mathrm{mM}$ EGTA $\left(\mathrm{R}_{\min }\right)$. $\mathrm{K}_{\mathrm{d}}$, fura-2 was assumed as $225 \mathrm{nM}$ according to previous work (Lips \& Keller, 1998; Ladewig \& Keller, 2000).

In experiments where the fura dyes were loaded as AM-esters, the background fluorescence was not clearly determinable, therefore fluorescent changes in defined ROls are given in relative values $F / F_{0}$, where $F_{0}$ is the fluorescence before stimulus or drug application. However, the conversion of ratio values into $\mathrm{Ca}^{2+}$ concentrations was approached by the following protocol. $R_{\max }$ and $R_{\min }$ were determined by exposing the AM-ester stained slice to $15 \mu \mathrm{M}$ ionomycin either in the presence of 
aCSF containing $10 \mathrm{mM} \mathrm{Ca}^{2+}\left(\mathrm{R}_{\max }\right)$ or $0 \mathrm{mM} \mathrm{Ca}^{2+}$ and $10 \mathrm{mM}$ EGTA $\left(\mathrm{R}_{\min }\right)$. Ionomycin is a $\mathrm{Ca}^{2+}$ ionophore leading to equalization of intra- and extracellular $\left[\mathrm{Ca}^{2+}\right]$. Then, the equation given above was applied.

\subsection{Analysis of calcium dynamics - the linear compartment model}

Somatic $\mathrm{Ca}^{2+}$ dynamics of hypoglossal motoneurons can be analysed according to a linear one-compartment model that has been described previously (Neher \& Augustine, 1992; Neher, 1995; Lips \& Keller, 1998, 1999). In this model, a single "effective" extrusion rate $\gamma$ is assumed, which is justified if somatic $\mathrm{Ca}^{2+}$ transients are described by a single exponential function. A second important parameter of the model is the "endogenous" $\mathrm{Ca}^{2+}$ buffering of the cell, which is quantified by the binding capacity $\kappa_{\mathrm{S}}$. Both parameters can be determined by loading cells with a buffer with known buffering properties, i.e. the indicator dye fura-2. For a given concentration of the indicator dye, the corresponding "exogenous" binding capacity $\kappa_{\mathrm{B}}$ can be calculated from the equation

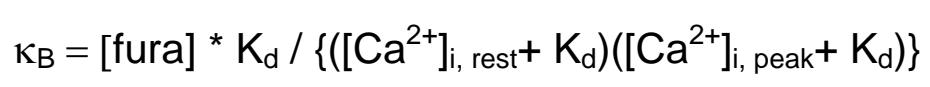

where $\left[\mathrm{Ca}^{2+}\right]_{i}$, rest, $\left[\mathrm{Ca}^{2+}\right]_{i}$, peak and $\mathrm{K}_{\mathrm{d}}$ denote resting, peak calcium concentration and the dissociation constant of the indicator dye, respectively. The decay time constant ( $\tau$ ) of a given $\mathrm{Ca}^{2+}$ transient is then described by the equation $\quad \tau=\left(\kappa_{\mathrm{S}}+\kappa_{\mathrm{B}}+1\right) / \gamma$.

Previous studies have established $\kappa_{S}=41 \pm 12$ for hypoglossal motoneurons (Lips \& Keller, 1998). $\gamma$ was calculated in the range of $131 \mathrm{~s}^{-1}$ to $35 \mathrm{~s}^{-1}$ for amplitudes between 60 nM and 476 nM, respectively (Lips \& Keller, 1999).

\subsection{Estimation of $\mathrm{Ca}^{2+}$ clearance rates using polynomial fits}

Analysing $\mathrm{Ca}^{2+}$ dynamics according to the one-compartment model neglects nonlinearities in $\mathrm{Ca}^{2+}$ clearance mechanisms. Therefore, for estimation of total and mitochondrial $\mathrm{Ca}^{2+}$ clearance rates, decay kinetics of $\mathrm{Ca}^{2+}$ transients were fitted with polynomial functions and further analysed as follows. 1) The decay of depolarisationevoked $\mathrm{Ca}^{2+}$ transients before and after FCCP incubation was fitted over 25-30 s from the first point of decay on with a polynomial function of $12^{\text {th }}$ order, yielding the 
most accurate fit. 2) The numerical derivative $\left(\mathrm{d}\left[\mathrm{Ca}^{2+}\right]_{i} / \mathrm{dt}\right)$ was calculated from the fit. 3) $-d\left[\mathrm{Ca}^{2+}\right] / / d t$ was plotted as a function of the $\left[\mathrm{Ca}^{2+}\right]_{i}$ values obtained from the fit. 4) Plots from transients with similar amplitudes were pooled ( $n=3$ for control and FCCP) and described by a polynomial function of seventh order. 5) The resulting polynomial fits -one for each condition- were then plotted as a function of the transient amplitude. 6) Finally, the fit calculated from the FCCP data was subtracted from the fit calculated for the control condition yielding the mitochondrial clearance rate $(\mu \mathrm{M} / \mathrm{s})$ as a function of $\Delta\left[\mathrm{Ca}^{2+}\right]_{\text {. }}$

\subsection{Monitoring of mitochondrial parameters}

Mitochondrial membrane potential $(\Delta \psi)$ was monitored using rhodamine 123 (rhod123). Rhod123 is a positively charged molecule and therefore accumulates within the negatively charged mitochondrial matrix, where its fluorescence is quenched. Upon depolarisation of $\Delta \psi$, rhod123 is released into the cytosol and its fluorescence increases. Because rhod123 is a non-ratiometric dye, fluorescence intensities are given in relative values $F / F_{0}$. In patch-clamp experiments, rhod123 was added to the pipette solution at $10 \mu \mathrm{g} / \mathrm{ml}$ according to a method described by Schuchmann et al. (2000). In other experiments, the slice was incubated with 10 $\mu \mathrm{g} / \mathrm{ml}$ rhod123 for 10 minutes followed by an intensive wash.

Mitochondrial metabolism (respiratory chain activity) was monitored by the intrinsic $\mathrm{NADH}$ fluorescence of motoneurons. NADH is the substrate for complex I of the mitochondrial respiratory chain (compare Fig. 1.4), therefore an increase in respiratory chain activity is represented by a decrease in the NADH fluorescence intensity and vice versa. NADH was excited at 360 and emitted fluorescence was collected using a dichroic mirror with mid-reflection at $425 \mathrm{~nm}$.

\subsection{Multiphoton imaging of intrinsic NADH fluorescence}

In experiments performed at the Dept. of Applied \& Engineering Physics, Cornell University, U.S.A. (head Prof. Dr. W. W. Webb), the intrinsic NADH fluorescence of MNs in the slice preparation was monitored using two-photon microscopy. This approach allowed for a higher spatial resolution of cellular structures in the slice as compared to CCD camera imaging. Also advantageous was the fact that two-photon 
microscopy restricts the excitation volume to the focus level $(<1 \mu \mathrm{m})$, so that no background fluorescence occurs. In the experimental set-up used, the laser beam was provided by a titanium-saphir-laser system (Spectra Physics, Mountain View, CA, U.S.A.), directed into a Bio-Rad MRC-600 scanning box with laboratory-built detection systems mounted on an Olympus BX50 upright microscope equipped with infra-red compatible dipping objectives (40x NA0.8 or 60x NA 0.9 ). The fluorescence emitted was filtered by a BGG22 filter set (Chroma, Rockingham, VT, U.S.A.) and detected by a photomultiplier tube (Hamamatsu, Bridgewater, NJ, U.S.A.). For excitation of $\mathrm{NADH}$, wavelengths in the range of $740-760 \mathrm{~nm}$ were employed. In timeseries, dynamic changes were evaluated in defined ROls using ImageJ software (NIH, Bethesda, MD, U.S.A.).

\subsection{Cytochrome-oxidase histochemistry}

Cytochrome-oxidase histochemistry allows assessment of the respiratory chain activity in fixed tissue, i.e. brain slices (Wong-Riley, 1989). Deeply anaesthetized mice were perfusion-fixed with warm $\left(37^{\circ} \mathrm{C}\right)$ phosphate buffered saline $\mathrm{pH} 7.4$ (PBS: $0.1 \mathrm{M} \mathrm{Na}_{2} \mathrm{HPO}_{4}, 0.1 \mathrm{M} \mathrm{NaH}_{2} \mathrm{PO}_{4}, 0.9 \% \mathrm{NaCl}$ ) followed by cold $\left(4^{\circ} \mathrm{C}\right)$ PBS containing $4 \%$ paraformaldehyde and $4 \%$ sucrose. Brains were dissected and postfixed in $4 \%$ paraformaldehyde $/ 4 \%$ sucrose in PBS for 4 hours at $4^{\circ} \mathrm{C}$. Brains were transferred to $20 \%$ sucrose in PBS for 1-2 days (until brains sank). Brains were embedded in OCT freezing compound and shock-frozen (30 seconds) in chilled 2-methylbutane (isopentane), which was cooled down in liquid nitrogen. Frozen blocks were wrapped with aluminium foil and stored in a freezer $\left(-80^{\circ} \mathrm{C}\right)$ for $>10$ minutes. The tissue block was then equilibrated in a cold cryostat cabinet and tissue sections were cut at 12 $\mu \mathrm{m}$. Sections were mounted on plus-slides and incubated with sterile filtered cytochrome-oxidase histochemistry buffer (0.1 M PBS containing 25 mg DAB (3,3'diaminobenzidine), $10 \mathrm{mg}$ cytochrome $\mathrm{c}$ and $2 \mathrm{~g}$ sucrose per $50 \mathrm{ml}$ ) for $3-4 \mathrm{~h}$ in the dark. Sections were washed three times with cold 0.1 M PBS, air-dried and cover slipped. The dark reaction product is a measure of cytochrome-oxidase activity. 


\subsection{Materials}

All calcium indicator dyes, as well as mitotracker green, were purchased from Molecular Probes (Leiden, Netherlands). Tetrodotoxin citrate (TTX), 6-cyano-7nitroquinoxaline-2,3-dione disodium salt (CNQX) and D(-)-2-amino-5phosphonopentanoic acid (AP-5) were purchased from Tocris (Bristol, U.K.), all other chemicals were from Sigma (St. Louis, MO, U.S.A.) or Merck (Darmstadt, Germany). Stock solutions were prepared as follows: fura-2 pentapotassium salt was dissolved in aqua $_{\text {dest }}$ at 1-2 mM, fura-2 AM and fura-FF AM were dissolved in DMSO at $1 \mathrm{mM}$, rhod123 was dissolved in ethanol $(10 \mathrm{mg} / \mathrm{ml})$. Carbonyl cyanide 4-trifluoromethoxyphenylhydrazone (FCCP) was dissolved in ethanol at $10 \mathrm{mM}$. Sodium cyanide $(\mathrm{CN})$ and sodium azide were dissolved in aqua $\mathrm{dest}(2 \mathrm{M})$ and diluted to the final concentration immediately before the experiment.

\subsection{Statistical analysis}

If not indicated otherwise, values in the text are given as mean \pm standard error (SEM), error bars in figures represent SEM too. The significance after pharmacological intervention was calculated using student t-test. The significance of linear correlation coefficients $\left(r_{0}\right)$ was determined by calculation of the probability $\mathrm{p}\left(|\mathrm{r}| \geq \mathrm{r}_{0}\right)$ according to Taylor (1982). 


\section{RESULTS}

\subsection{Mitochondrial $\mathrm{Ca}^{2+}$ buffering in mouse hypoglossal motoneurons}

In this section, the contribution of mitochondria to regulation of intracellular $\mathrm{Ca}^{2+}$ homeostasis in hypoglossal motoneurons (MNs) will be investigated.

\subsubsection{Stimulation of $\mathrm{Ca}^{2+}$ transients in motoneurons loaded with fura-2/AM}

The first aim of the study was to test the role of mitochondria as $\mathrm{Ca}^{2+}$ buffer in MNs under physiological conditions. For this purpose, a protocol was established, which allowed monitoring of intracellular $\mathrm{Ca}^{2+}$ transients in MNs in brain slices without washing out intracellular structures, as might occur during patch-clamp recordings. This was important since cytosolic dialysis could disrupt cytoskeletal elements that establish mitochondrial localisation within a cell. Additionally, previous experiments indicated that the pattern of $\mathrm{Ca}^{2+}$ response following a defined activation protocol may differ between patch-clamped and ester-loaded cells (unpublished observation). Therefore an experimental protocol was developed, where motoneurons in brain slices were loaded with the membrane-permeable dye fura-2/AM. Fig. 3.1.1 shows such fura-2/AM loaded hypoglossal neurons, where differences in fluorescent intensity are largely explained by different depths of cells within the slice.

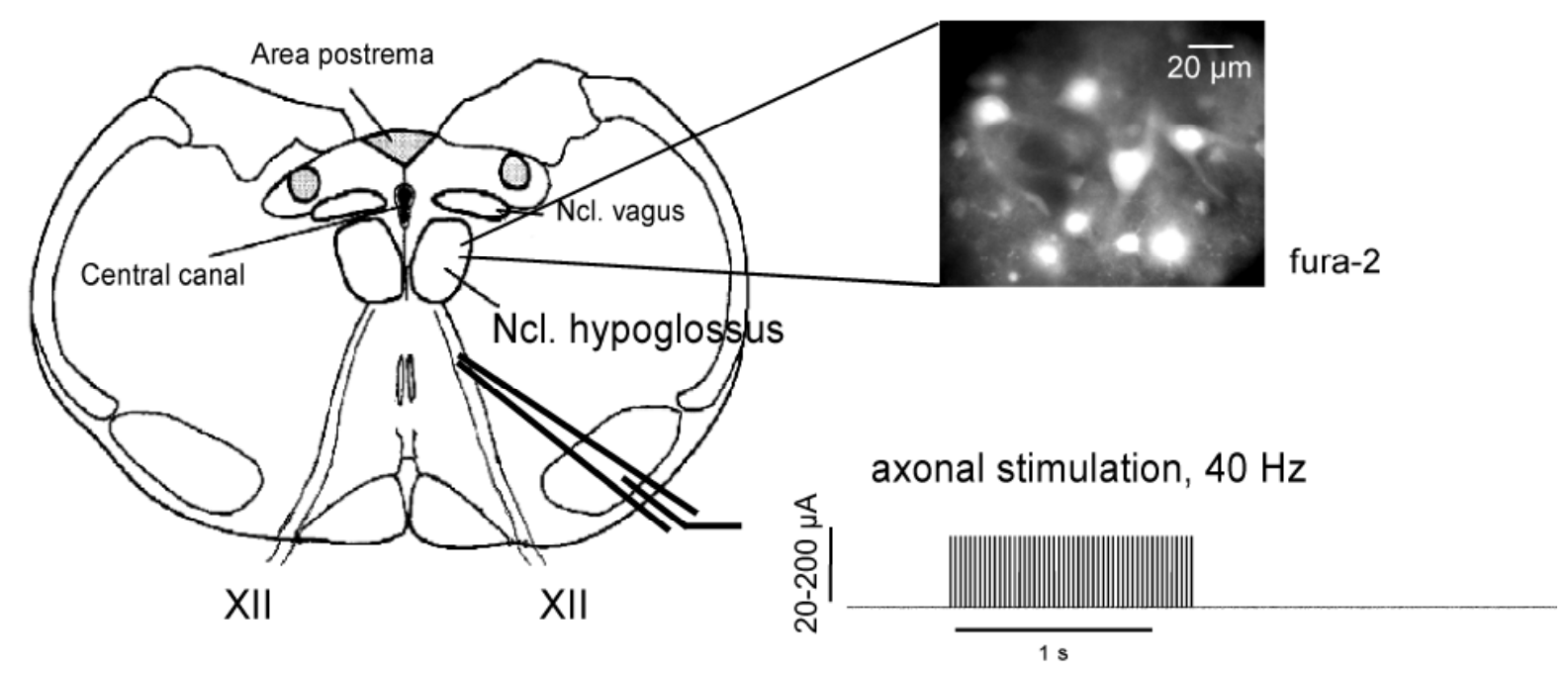

Fig. 3.1.1: Illustration of a mouse brain stem slice containing the nucleus (Ncl.) hypoglossus. On the right, a CCD camera image ( $4 \times 4$ binning) showing hypoglossal MNs loaded with fura-2 AM (excitation at $390 \mathrm{~nm}$ ). Antidromic activation of hypoglossal MNs in the slice was achieved by delivering short repetitive depolarising current pulses (single pulse 0.4-2 ms, 50-150 $\mu \mathrm{A}$ ) at $40 \mathrm{~Hz}$ via a glass pipette placed along the axonal pathway of the hypoglossal nucleus. Pulse protocol is shown at lower right. 
Besides the advantage of preventing "wash-out" of the cytosol by patch pipette dialysis, the protocol of AM-loading allowed me to utilize the specific anatomy of the system by selecting a cutting plane for brain stem slices that preserved a substantial fraction of the hypoglossal nerve. This permitted the selective activation of hypoglossal MNs by applying antidromic stimulation to their axons forming the hypoglossal nerve (Fig. 3.1.1). Furthermore, by using the previously established quantitative model of $\mathrm{Ca}^{2+}$ homeostasis (see Methods), it was possible to estimate the cytosolic fura-2 concentration in AM-loaded hypoglossal motoneurons without whole-cell recording. This was done by evaluating the recovery time constant $\tau$ of cytosolic $\mathrm{Ca}^{2+}$ transients after fitting with a single exponential function, which indicated cytosolic fura-2 concentrations in the range of 100 to $200 \mu \mathrm{M}$.

$A$

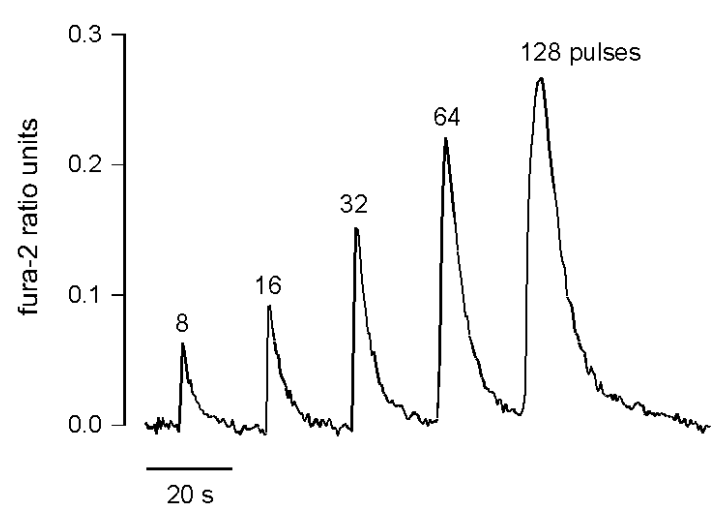

$B$

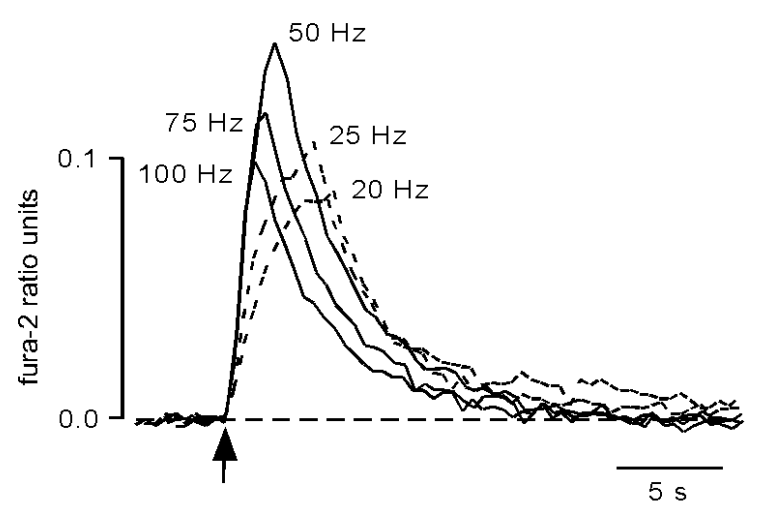

Fig. 3.1.2: Monitoring of $\left[\mathrm{Ca}^{2+}\right]_{i}(360 / 390 \mathrm{~nm}$ fluorescence ratio) within a region of interest drawn over the soma of a fura-2/AM loaded hypoglossal MN in the slice. $\boldsymbol{A}$ Increasing numbers of depolarising pulses evoke $\left[\mathrm{Ca}^{2+}\right]_{\mathrm{i}}$ transients with increasing amplitudes. 0.1 fura-2 ratio unit corresponds to $\sim 100$ $\mathrm{nM}$ elevation of $\left[\mathrm{Ca}^{2+}\right]_{\text {. }}$ B 50 stimuli were delivered at different stimulation frequencies. Note that the amplitude of $\left[\mathrm{Ca}^{2+}\right]_{i}$ elevation is dependent on the stimulation frequency with highest elevation around $50 \mathrm{~Hz}$. The arrow indicates onset of stimulation.

To establish reproducible recording conditions, first, control stimulation experiments were performed. Fig. 3.1.2 demonstrates that stimulation-evoked $\left[\mathrm{Ca}^{2+}\right]_{i}$ elevations were strongly correlated with the number of stimulation pulses applied. $\mathrm{Ca}^{2+}$ responses increased rapidly from a resting level of $\sim 0.1 \mu \mathrm{M}$ and displayed many similarities with transients observed during rhythmic respiratory-related activity in an intact neuronal network (Ladewig \& Keller, 2000). Maximum $\left[\mathrm{Ca}^{2+}\right]_{i}$ elevations for 128 stimuli were calculated as $\sim 300 \mathrm{nM}$. The amplitude of stimulation-evoked $\mathrm{Ca}^{2+}$ transients was also strongly correlated with the strength of current (20-200 $\mu \mathrm{A})$ applied (not shown). $\left[\mathrm{Ca}^{2+}\right]_{i}$ elevations were largest when choosing stimulation 
frequencies around $50 \mathrm{~Hz}$ (Fig. 3.1.2 B), which is close to the maximum steady state firing rate of hypoglossal MNs. Such data suggested that fura-2/AM loaded slices represent a reliable tool to study $\mathrm{Ca}^{2+}$ dynamics in hypoglossal MNs.

\subsubsection{Contribution of mitochondria to $\mathrm{Ca}^{2+}$ clearance}

To test the contribution of mitochondria to clearance of stimulation-induced $\mathrm{Ca}^{2+}$ transients in hypoglossal MNs, the protonophore p-trifluoromethoxy-phenylhydrazone (FCCP) was applied. This drug dissipates the proton gradient across the inner mitochondrial membrane leading to depolarisation of the mitochondria potential $(\Delta \psi)$. As $\mathrm{Ca}^{2+}$ uptake into mitochondria is thought to critically depend upon the potential gradient over the inner membrane, protonophores are considered to critically impair mitochondrial $\mathrm{Ca}^{2+}$ accumulation.

To see how FCCP acted in the slice preparation, $\Delta \psi$ of hypoglossal MNs was monitored in rhod123 stained slices and FCCP $(1 \mu \mathrm{M})$ was added (Fig. 3.1.3). The experiment showed that it took at least 3 minutes until robust mitochondrial depolarisation was achieved, thus, in following experiments slices were exposed to FCCP for at least 4 minutes.

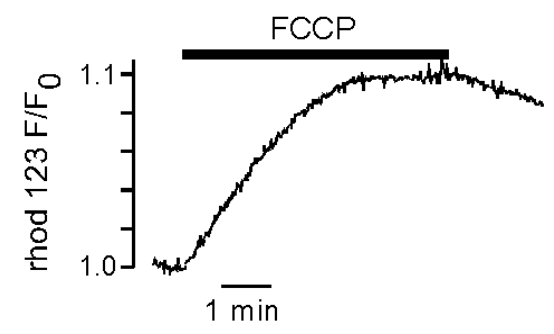

Fig 3.1.3: Monitoring of the mitochondrial potential $(\Delta \psi)$ over the soma of a hypoglossal MN bath loaded with rhod123. Addition of FCCP $(1 \mu \mathrm{M})$ increases rhod123 fluorescence $\left(F / F_{0}\right)$ excited at $485 \mathrm{~nm}$ indicating depolarisation of $\Delta \psi$. Maximum depolarisation of $\Delta \psi$ is achieved $\sim 4$ minutes after onset of incubation with FCCP.

Then, $\left[\mathrm{Ca}^{2+}\right]_{i}$ was monitored by the fura-2 360/390nm fluorescence ratio and a control stimulation was carried out to evoke a rise in $\left[\mathrm{Ca}^{2+}\right]_{i}$ before FCCP were washed-in (Fig. 3.1.4 A). The strength of stimulation was always chosen to evoke $\left[\mathrm{Ca}^{2+}\right]_{\mathrm{i}}$ transients with amplitudes between 50 and $200 \mathrm{nM}$ that resembled those observed in the rhythmic respiratory network (Lips \& Keller, 1999; Ladewig \& Keller, 2000). Oligomycin $(5 \mu \mathrm{g} / \mathrm{ml})$, a specific blocker of the mitochondrial $F_{1}, F_{0}$-ATP synthase, was added together with FCCP to prevent any accelerated consumption of cellular ATP by reverse mode operation of the ATP synthase (Budd \& Nicholls, 1996; Babcock et al., 1997; David et al., 1998). Consistent with previous observations (Babcock et al., 1997; Ladewig et al., 2003), FCCP induced an increase in resting $\left[\mathrm{Ca}^{2+}\right]_{i}$ of variable 
size and duration apparently reflecting release of stored $\mathrm{Ca}^{2+}$ out of mitochondria. This effect was clearly attributable to FCCP, because oligomycin alone did not affect resting $\left[\mathrm{Ca}^{2+}\right]_{\mathrm{i}}$ levels within the first 10 minutes of application (Fig. 3.1.4 B).
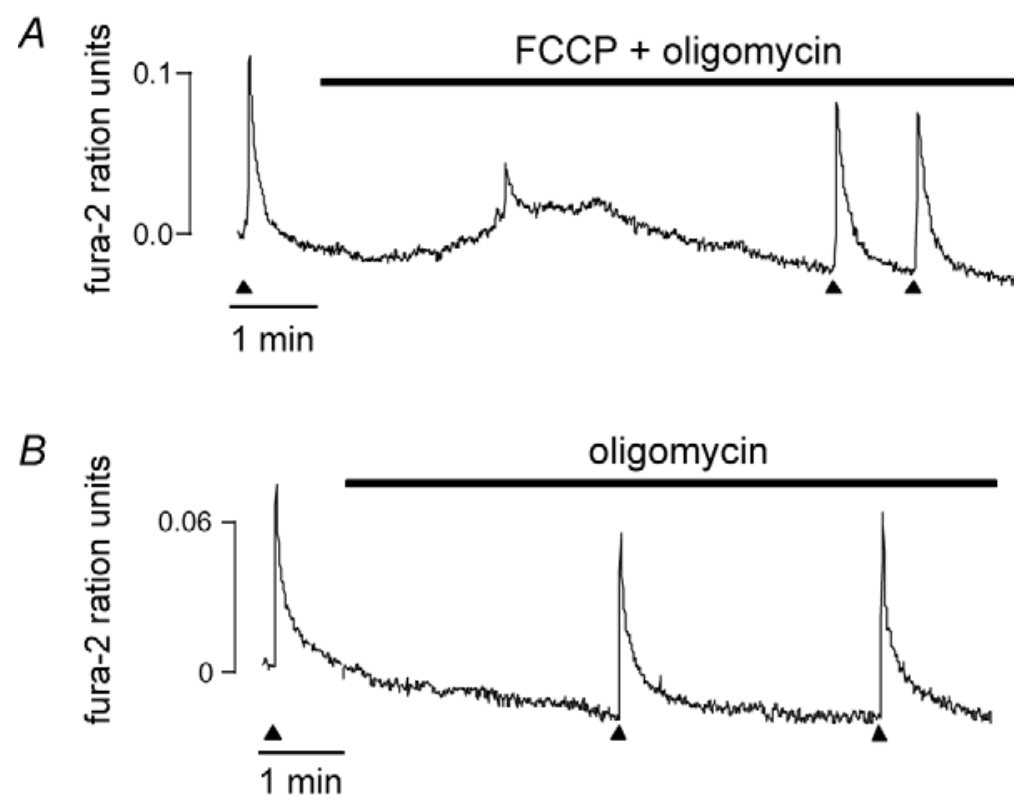

Fig. 3.1.4: Imaging of cytosolic $\left[\mathrm{Ca}^{2+}\right](360 / 390 \mathrm{~nm}$ fluorescence ratio) in hypoglossal MNs loaded with fura-2/AM. Antidromic stimulation ( $\mathbf{\Lambda}$ ) induces cytosolic $\mathrm{Ca}^{2+}$ transients. $\boldsymbol{A}$ FCCP $(1 \mu \mathrm{M})$ was added to depolarise mitochondria as indicated. Oligomycin $(5 \mu \mathrm{g} / \mathrm{ml})$ was added in addition to prevent accelerated ATP consumption. Note that FCCP reversibly increased resting $\left[\mathrm{Ca}^{2+}\right]_{\mathrm{i}}(\boldsymbol{A})$, as this was not seen when oligomycin $(5 \mu \mathrm{g} / \mathrm{ml})$ was added alone $(\boldsymbol{B})$.

When voltage-dependent $\mathrm{Ca}^{2+}$ influx was then stimulated in the presence of FCCP and oligomycin, the decay time constant tau $(\tau)$ of stimulus-evoked $\left[\mathrm{Ca}^{2+}\right]_{\mathrm{i}}$ transients was prolonged to $1.92 \pm 0.13$ times the value obtained for control elevations with mean amplitudes of $\sim 120 \mathrm{nM}(\tau=2.38 \pm 0.26 \mathrm{~s}$ before and $4.39 \pm 0.42 \mathrm{~s}$ after addition of drugs; $p<0.01 ; n=17$; Fig. 3.1.5 $A$ and $C$ ). Since application of oligomycin alone did not affect the recovery time $(\tau=1.99 \pm 0.19 \mathrm{~s}$ and $1.71 \pm 0.09 \mathrm{~s}$ before and after addition of oligomycin alone; $p>0.05 ; n=8$; Fig $3.1 .5 B$ and $C$ ), the effect of FCCP is clearly attributable to collapse of $\Delta \psi$, and not to inhibition of ATP-synthesis. The peak amplitude of $\mathrm{Ca}^{2+}$ transients was not significantly altered by FCCP (Fig. 3.1.5 D; relative change from $1 \pm 0.11$ to $1.16 \pm 0.07$ after addition of FCCP and oligomycin; $p>0.05$ ) nor by oligomycin (relative change from $1 \pm 0.12$ to $1.15 \pm 0.11$ after addition of oligomycin alone; $p>0.05)$.

These results were almost undistinguishable from the data obtained on patchclamped hypoglossal MNs in the same preparation using the same indicator dye, 
where the protonophore CCCP prolonged the recovery time of $\mathrm{Ca}^{2+}$ transients 1.94 fold without significantly altering in the transient amplitude (Ladewig et al., 2003).

$A$

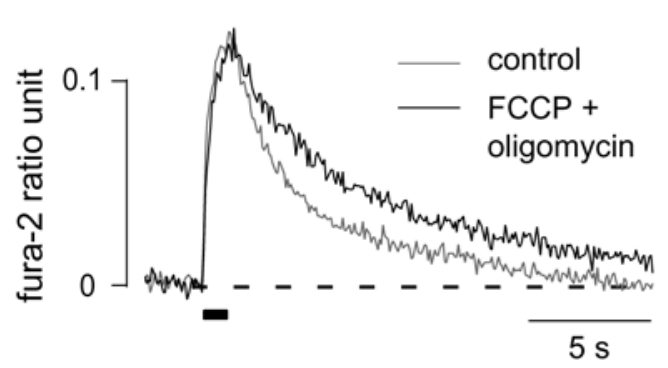

$C$

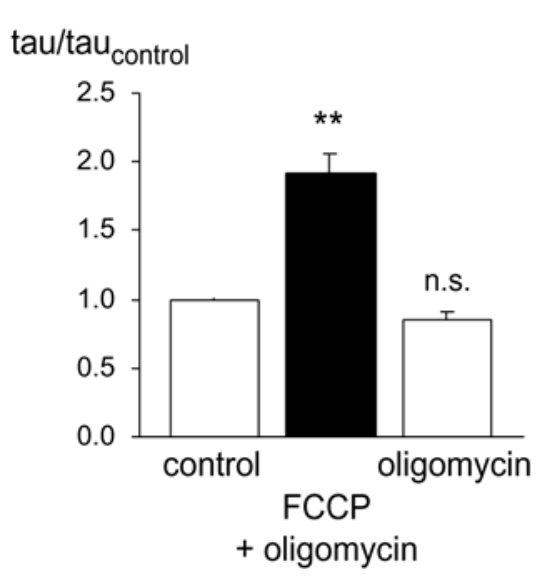

$B$

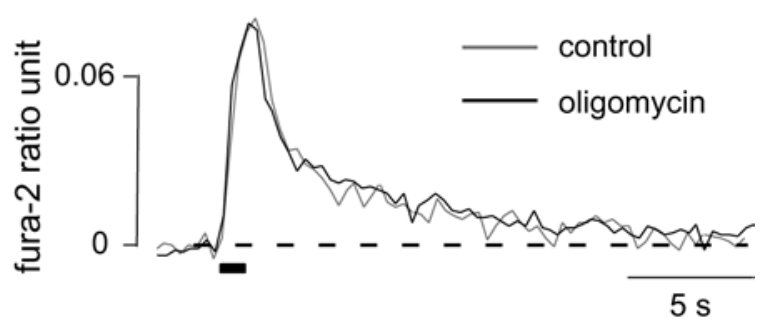

$D \quad \Delta\left[\mathrm{Ca}^{2+}\right]_{\mathrm{i}} / \Delta\left[\mathrm{Ca}^{2+}\right]_{\text {icontrol }}$

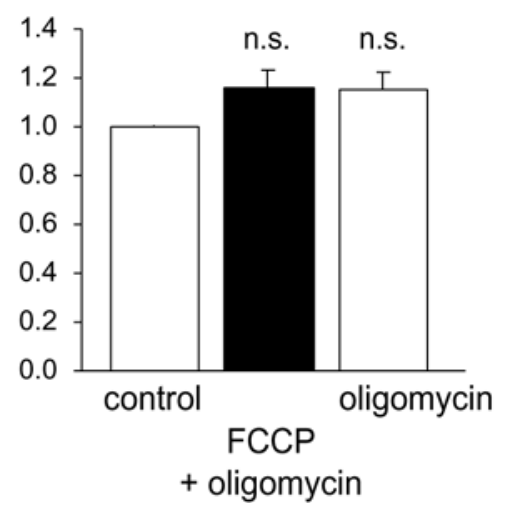

Fig. 3.1.5: Stimulation-evoked $\left[\mathrm{Ca}^{2+}\right]_{i}$ transients before and $10 \mathrm{~min}$ after addition of FCCP and oligomycin $(\boldsymbol{A})$ and after addition of oligomycin alone $(\boldsymbol{B})$. The black bars indicate duration of stimulation. Note the different acquisition rates of $10 \mathrm{~Hz}(\mathrm{~A})$ and $3 \mathrm{~Hz}(\mathrm{~B})$. C, $\boldsymbol{D}$ Graphical summary of the effect of FCCP and oligomycin on the decay time constant (tau) and amplitude $\left(\Delta\left[\mathrm{Ca}^{2+}\right]_{\mathrm{i}}\right)$ of stimulation-induced $\mathrm{Ca}^{2+}$ transients. Note that neither the amplitude nor the decay of stimulation induced $\mathrm{Ca}^{2+}$ transients is significantly affected by oligomycin alone $(n=8)$, whereas FCCP prolongs the recovery time of $\mathrm{Ca}^{2+}$ transients to $1.92 \pm 0.13$ fold the control value without altering the amplitude $(n=17)$.

To better characterize mitochondrial $\mathrm{Ca}^{2+}$ handling, the time integral of stimulusevoked $\left[\mathrm{Ca}^{2+}\right]_{i}$ transients $\left(\Delta\left[\mathrm{Ca}^{2+}\right]_{i}{ }^{*} \tau\right)$ was also considered. Most important, increases in $\left[\mathrm{Ca}^{2+}\right]_{i}$ integrals after FCCP application provide an estimate for the total amount of $\left[\mathrm{Ca}^{2+}\right]_{i}$ that is buffered by mitochondria. In the experiments, these drugs led to a $2.27 \pm 0.25$-fold increase in the $\left[\mathrm{Ca}^{2+}\right]_{\mathrm{i}}$ integral, indicating that $\sim 50 \%$ of depolarisation-induced $\mathrm{Ca}^{2+}$ influx is taken up by mitochondria in the undisturbed cell, a value that can also be assumed for patch-clamped motoneurons. 
Results

\subsubsection{Temperature dependence of mitochondrial $\mathrm{Ca}^{2+}$ buffering}

In the next experiments, the temperature dependence of mitochondrial $\mathrm{Ca}^{2+}$ buffering was tested. So far, all studies investigating mitochondrial $\mathrm{Ca}^{2+}$ buffering in $\mathrm{MN}$ somata were performed at room temperature (Carriedo et al., 2000; Ladewig et al., 2003) thus neglecting the potential influence of temperature on mitochondrial mechanisms, which has i.e. been demonstrated by David \& Barrett (David \& Barrett, 2000).

$A$
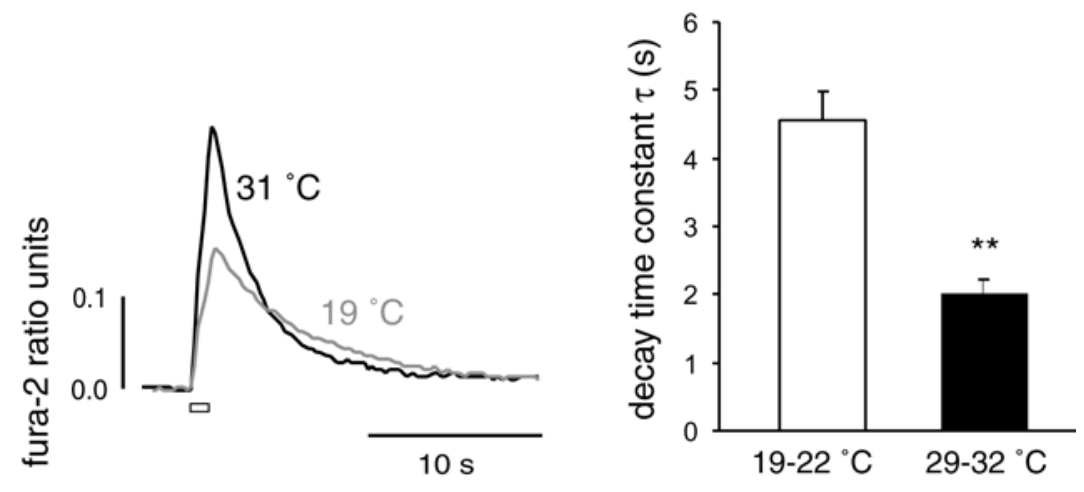

B
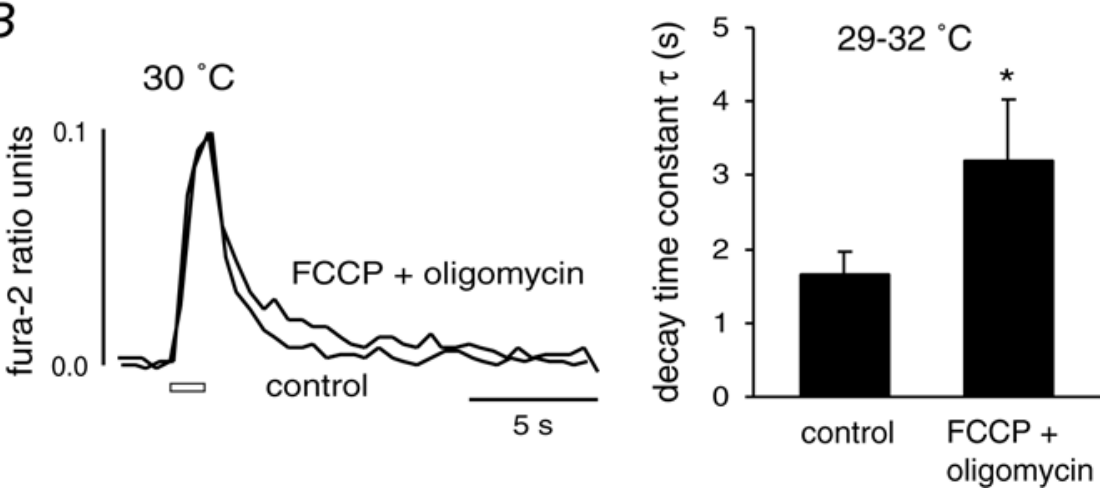

Fig. 3.1.6: $\boldsymbol{A}$ Imaging of $\left[\mathrm{Ca}^{2+}\right]_{i}$ by the $360 / 390 \mathrm{~nm}$ fluorescence ratio in a hypoglossal MN loaded with fura-2/AM. $\mathrm{Ca}^{2+}$ transients were elicited by antidromic stimulation (open bar) first at room temperature $\left(19^{\circ} \mathrm{C}\right)$, then after increasing the bath temperature to $31^{\circ} \mathrm{C}$. The temperature change reduced the decay time constant $(\tau)$ of stimulation-induced $\mathrm{Ca}^{2+}$ transients to $0.48 \pm 0.07$ control $(p<0.01, n=17)$. $B$ At near physiological temperatures $\left(29-32^{\circ} \mathrm{C}\right)$, application of FCCP $(1 \mu \mathrm{M})$ in the presence of oligomycin $(5 \mu \mathrm{g} / \mathrm{ml})$ prolonged the decay time constant $(\tau)$ of stimulation-induced $\mathrm{Ca}^{2+}$ transients to $1.81 \pm 0.22$ control $(p<0.04, n=11)$. Thus, mitochondria buffer a similar fraction of $\mathrm{Ca}^{2+}$ load at near physiological temperatures compared to room temperature (compare to Fig. 3.1.5 A), but the rate of mitochondrial $\mathrm{Ca}^{2+}$ uptake was significantly speeded up at higher temperatures.

Stimulation-induced $\mathrm{Ca}^{2+}$ transients in fura-2/AM loaded hypoglossal MNs displayed significantly faster recovery times at $29-32^{\circ} \mathrm{C}$ compared to room temperature (19$21^{\circ} \mathrm{C}$ ). This could be seen, when gradually increasing the temperature over a period of $\sim 5 \mathrm{~min}$ and analysing stimulation-induced $\mathrm{Ca}^{2+}$ transients before and after 
temperature change as demonstrated in Fig. 3.1.6 $A$ (reduction of tau $(\tau)$ to $0.48 \pm$ 0.07 control; $n=17 ; \mathrm{p}<0.01)$. At the same time, the amplitude of $\mathrm{Ca}^{2+}$ transients increased to $1.82 \pm 0.19$ fold control $(p<0.01)$. Fig. 3.1.6 B illustrates the effect of mitochondrial depolarisation on stimulation-induced $\mathrm{Ca}^{2+}$ transients at $29-32^{\circ} \mathrm{C}$. Comparable to the previous experiment at room temperature (Fig. 3.1.5 A), incubation with $1 \mu \mathrm{M}$ FCCP together with oligomycin $(5 \mu \mathrm{g} / \mathrm{ml})$ for more than 4 minutes prolonged the recovery time of stimulation-induced $\mathrm{Ca}^{2+}$ transients to $1.81 \pm$ 0.22 fold control $(p<0.04 ; n=11)$, but had no significant effect on the transient amplitude. This indicated that the fraction of $\mathrm{Ca}^{2+}$ taken up by mitochondria near physiological temperatures does not significantly differ from the one at room temperature. However, as the recovery time of $\mathrm{Ca}^{2+}$ transients was more than doubled for a temperature increase of $10{ }^{\circ} \mathrm{C}$, it was concluded that mitochondrial $\mathrm{Ca}^{2+}$ uptake must also have speeded up $\sim 2$ fold $(\mathrm{Q} 10=2.1)$ to yield the same relative contribution. Taken together, the results therefore support the assumption that mitochondrial $\mathrm{Ca}^{2+}$ uptake is highly temperature dependent in mouse hypoglossal MN somata.

\subsubsection{Impact of mitochondrial $\mathrm{Ca}^{2+}$ buffering on voltage-activated $\mathrm{Ca}^{2+}$ currents}

A surprising finding of this study and corresponding experiments on patch-clamped MNs (Ladewig et al., 2003) was that inhibition of mitochondrial $\mathrm{Ca}^{2+}$ uptake prolonged the recovery time but did not significantly alter the amplitude of voltageactivated $\mathrm{Ca}^{2+}$ transients (Fig. 3.1.5). This was unexpected, because studies on cultured MNs and motor nerve terminals showed a large increase in the amplitude of $\mathrm{Ca}^{2+}$ transients as a result of mitochondrial inhibition (Carriedo et al., 2000; David \& Barrett, 2000). Therefore, the possibility was considered that FCCP by itself or the lack of mitochondrial $\mathrm{Ca}^{2+}$ buffering as a consequence of FCCP action might influence voltage-activated $\mathrm{Ca}^{2+}$ influx in a way similar to that described for chromaffin cells (Hernandez-Guijo et al., 2001).

To test this issue, hypoglossal MNs were patch-clamped in the slice and $\mathrm{Ca}^{2+}$ currents were elicited by repetitive $200 \mathrm{~ms}$ depolarisations from $-60 \mathrm{mV}$ to $0 \mathrm{mV}$ (every 20 s). Like it has been described in previous studies (Powers \& Binder, 2003), different patterns of $\mathrm{Ca}^{2+}$ current activation - apparently with and without a contribution of dendritic channels - could be observed. Peak $\mathrm{Ca}^{2+}$ current amplitude 
displayed a considerable run down over time (Fig. 3.1.7 A). Mitochondrial $\mathrm{Ca}^{2+}$ uptake was then inhibited by addition of $1 \mu \mathrm{M}$ FCCP, but without significantly altering the course of current decline as compared to the control population (control population $n=7$; treated with FCCP $n=6$ ). FCCP did not significantly change the inactivation time constant $(\tau)$ of depolarisation-evoked $\mathrm{Ca}^{2+}$ currents either (Fig. 3.1.7 $B) . \mathrm{Ca}^{2+}$ currents were recorded in the presence of FCCP over a period of 6-7 minutes, where robust mitochondrial depolarisation has to be expected (compare Fig. 3.1.3) and therefore any significant change in current amplitude or inactivation due to disturbed mitochondrial $\mathrm{Ca}^{2+}$ buffering would have been detected.

$A$

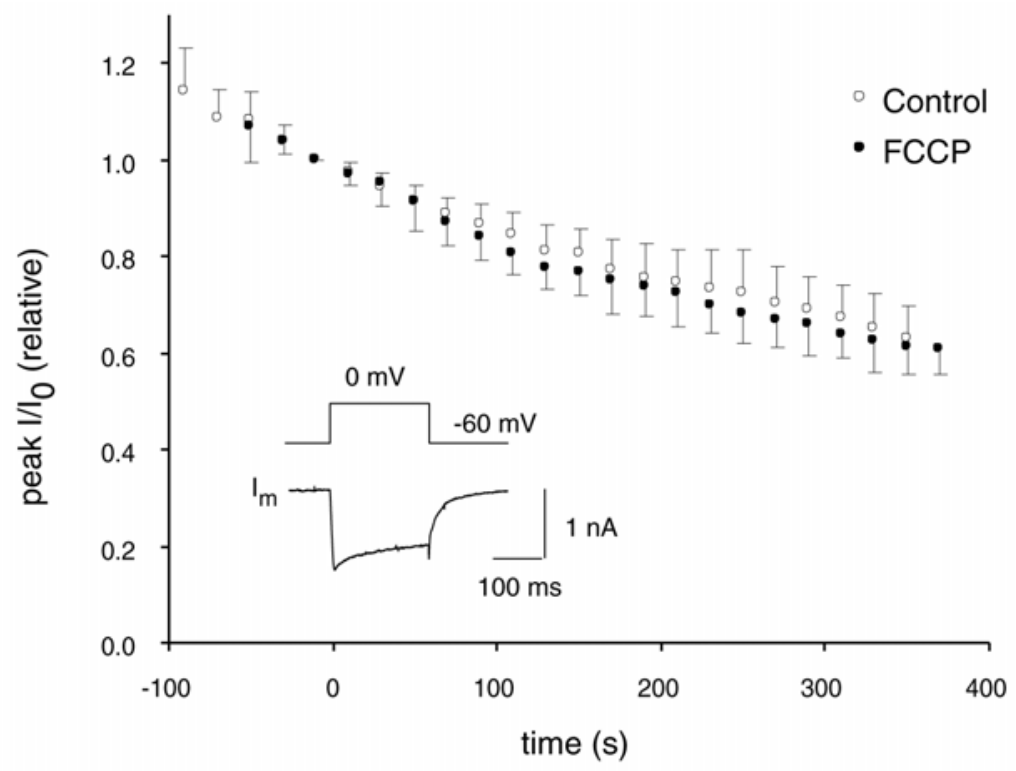

$B$

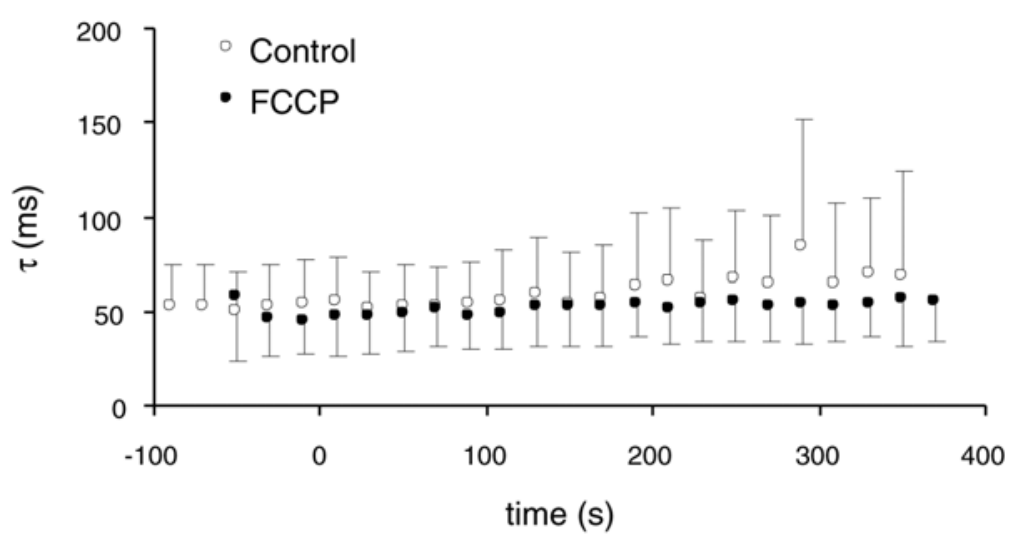

Fig. 3.1.7: $\boldsymbol{A}$ Whole cell $\mathrm{Ca}^{2+}$ currents were elicited in patch-clamped hypoglossal MNs by repetitive (every $20 \mathrm{~s}$ ) step depolarisation from a holding potential of $-60 \mathrm{mV}$. The inset shows a representative $\mathrm{Ca}^{2+}$ current and the pulse protocol. The graph plots relative peak currents (mean \pm standard deviation) of motoneurons under control conditions (open circles, $n=7)$ or treated with FCCP $(1 \mu \mathrm{M}$, filled circles, $n=6$ ). $I_{0}$ is the current at beginning of FCCP application. Note that FCCP does not significantly affect the decline of $\mathrm{Ca}^{2+}$ currents over time. $B$ Inactivation time constants $(\tau)$ of $\mathrm{Ca}^{2+}$ currents (mean \pm standard deviation) of the same cell population analysed in A. FCCP was added at time point 0 (filled circles) with no significant effect on the course of $\mathrm{Ca}^{2+}$ current inactivation compared to control cells (open circles). 


\subsubsection{Impact of dye affinity}

Next, the possibility was considered that the lacking effect of FCCP on the amplitude of $\mathrm{Ca}^{2+}$ transients was attributable to the low affinity indicator dye fura-2. This was conceivable since in those studies where FCCP dramatically increased the amplitude of $\mathrm{Ca}^{2+}$ transients, dyes with much lower affinity (fura-2FF and Oregon green BAPTA $5 N$ ) had been used.

To test this possibility slices were loaded with fura-FF $(\mathrm{Kd} \sim 5.5 \mu \mathrm{M})$, which displays a $\sim 6$ fold lower $\mathrm{Ca}^{2+}$ buffering capacity $\left(\kappa_{\mathrm{B}}\right)$ for $\left[\mathrm{Ca}^{2+}\right]_{i}$ elevations below $500 \mathrm{nM}$ compared to fura-2. AM-loading and stimulation of $\mathrm{Ca}^{2+}$ transients were performed as described for fura-2/AM. As expected, decay time constants $(\tau)$ of stimulationinduced $\mathrm{Ca}^{2+}$ transients were significantly faster when fura-FF was used $(\tau=0.9 \pm$ $0.1 \mathrm{~s} ; \mathrm{n}=12$; mean amplitudes $\sim 280 \mathrm{nM})$ compared to values for fura-2 $(\tau=2.4 \pm$ $0.3 \mathrm{~s} ; \mathrm{n}=17 ; \mathrm{p}<0.01)$ and almost reached the value of $0.7 \pm 0.2 \mathrm{~s}$ that was calculated for hypoglossal MNs under undisturbed conditions (Lips \& Keller, 1998).
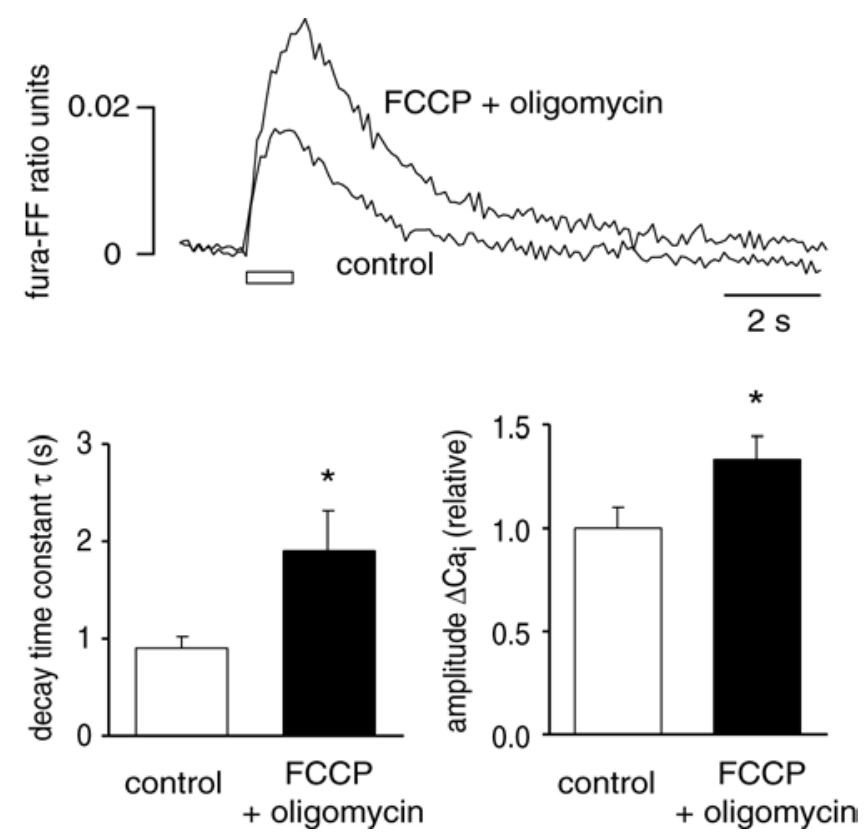

Fig. 3.1.8: Imaging of $\left[\mathrm{Ca}^{2+}\right]_{i}(360 / 390 \mathrm{~nm}$ fluorescence ratio) in a hypoglossal MN loaded with furaFF/AM. $\mathrm{Ca}^{2+}$ transients were elicited by antidromic stimulation (open bar). A change in the fura-FF ratio of 0.03 corresponds to a change in $\left[\mathrm{Ca}^{2+}\right]_{\mathrm{i}}$ of $\sim 500 \mathrm{nM}$. Incubation with FCCP $(1 \mu \mathrm{M})$ in the presence of oligomycin $(5 \mu \mathrm{g} / \mathrm{ml})$ for 4-8 min prolonged the recovery time of voltage-activated $\mathrm{Ca}^{2+}$ transients to $2 \pm 0.36$ fold control $(n=12, p=0.02)$. The peak amplitude was increased to $1.33 \pm 0.11$ fold control $(p<0.05)$. Note that this increase in amplitude was not seen when fura- 2 was used as indicator dye (Fig. 3.1.5). 
Fura-FF loaded MNs were then incubated with FCCP $(1 \mu \mathrm{M})$ and oligomycin (5 $\mu \mathrm{g} / \mathrm{ml}$ ) for 6-8 min to depolarise mitochondria. As illustrated in Fig. 3.1.8, this prolonged the recovery time of voltage-activated $\mathrm{Ca}^{2+}$ transients to $1.9 \pm 0.4 \mathrm{~s}$, which corresponds to a 2.08 fold prolongation compared to the control value $(n=12$ cells, 7 slices, $p=0.02)$. The magnitude of prolongation was comparable to the experiments using fura-2 (see Fig. 3.1.5). In contrast, the peak amplitudes of $\mathrm{Ca}^{2+}$ transients in fura-FF loaded cells were significantly increased to $1.33 \pm 0.11$ fold control after FCCP incubation $(p<0.05)$, an effect that was not observed when fura-2 was employed. This indicated that the choice of the $\mathrm{Ca}^{2+}$ indicator dye and its affinity may influence evaluation of $\mathrm{Ca}^{2+}$ transient amplitudes and possible mechanisms for the observed phenomenon will be discussed below.

\subsubsection{Quantification of mitochondrial $\mathrm{Ca}^{2+}$ clearance rate}

To obtain more detailed information about mitochondrial $\mathrm{Ca}^{2+}$ handling in hypoglossal MNs, the attempt was made to quantify the rate of mitochondrial $\mathrm{Ca}^{2+}$ clearance in a way similar to previous studies (Herrington et al., 1996; Fierro et al., 1998). For maximal control of intracellular parameters, in particular the concentration of indicator dye, it was decided to evaluate clearance rates on patch clamped $\mathrm{MNs}$, where $\mathrm{Ca}^{2+}$ transients were elicited by depolarisation to $0 \mathrm{mV}$ from a holding potential of $-60 \mathrm{mV}$. A CsCl based intracellular solution was chosen and $0.5 \mu \mathrm{M}$ TTX, $10 \mathrm{mM}$ TEA and 10 $\mathrm{nM}$ apamin were added to the CSF for simultaneous monitoring of $\mathrm{Ca}^{2+}$ currents. Although decay kinetics of voltage-activated $\mathrm{Ca}^{2+}$ transients up to $0.5 \mu \mathrm{M}$ were appropriately described by single exponential functions, a more exact fitting was achieved using polynomial functions, indicating that clearance mechanisms are not entirely linear in the tested $\left[\mathrm{Ca}^{2+}\right]_{i}$ range. Polynomial fitting was therefore applied for estimation of total and mitochondrial clearance rates.

Experiments were performed, where 3-4 depolarising voltage steps of increasing duration (50 - $950 \mathrm{~ms}$ ) were applied every $40 \mathrm{~s}$ to obtain $\mathrm{Ca}^{2+}$ transients with increasing amplitudes. Mean resting $\left[\mathrm{Ca}^{2+}\right]_{i}$ was measured as $\sim 90 \mathrm{nM}(\mathrm{n}=6)$. Then, 1 $\mu \mathrm{M}$ FCCP was added to depolarise mitochondria and 4 minutes later, the voltage step protocol was repeated. At that time, resting $\left[\mathrm{Ca}^{2+}\right]_{i}$ was slightly elevated by $39 \pm$ $10 \mathrm{nM}(\mathrm{n}=6)$ compared to the control condition. For calculation of total and mitochondrial $\mathrm{Ca}^{2+}$ clearance rates, $\mathrm{Ca}^{2+}$ transients with amplitudes of 0.3-0.4 $\mu \mathrm{M}$ 
before and after addition of FCCP were selected and the decay was fitted with a polynomial function of $12^{\text {th }}$ order (Fig. 3.1.9 A). The polynomial fits were then used to calculate clearance rates as a function of $\Delta\left[\mathrm{Ca}^{2+}\right]_{i}$ as described in the methods section. Finally, the rates of $\mathrm{Ca}^{2+}$ clearance were corrected for $\mathrm{Ca}^{2+}$ buffering by cytosol and added indicator dye (100 $\mu \mathrm{M}$ fura-2). This was done by multiplying with the binding ratio of the cytosol, $\kappa_{\mathrm{S}}=41$ (Lips \& Keller, 1998), and the binding constant of fura- $2, \kappa_{\mathrm{B}}$. The latter one was given by the equation

$$
\kappa_{B}=[\text { fura }]{ }^{*} K_{d} /\left\{\left(\left[\mathrm{Ca}^{2+}\right]_{i, \text { rest }}+\mathrm{K}_{d}\right)\left(\left[\mathrm{Ca}^{2+}\right]_{\mathrm{i}, \text { peak }}+\mathrm{K}_{\mathrm{d}}\right)\right\}
$$

assuming $\mathrm{K}_{\mathrm{d}, \text { fura- } 2}=0.22 \mu \mathrm{M},\left[\mathrm{Ca}^{2+}\right]_{\mathrm{i} \text {, rest }}=0.1 \mu \mathrm{M}$ and $\left[\mathrm{Ca}^{2+}\right]_{\mathrm{i}, \text { peak }}=\left(0.1 \mu \mathrm{M}+\Delta\left[\mathrm{Ca}^{2+}\right]_{\mathrm{i}}\right)$. In Fig. 3.1.9 $B$, the buffer-corrected clearance rates as a function of $\Delta\left[\mathrm{Ca}^{2+}\right]_{i}$ are displayed. The average clearance rate constant under undisturbed conditions $\left(\gamma_{\text {total }}\right)$, which is represented by the slope of the control trace, is $152 \mathrm{~s}^{-1}$; the average mitochondrial clearance rate constant $\left(\gamma_{\text {mito }}\right)$ is $70 \mathrm{~s}^{-1}$.

$A$

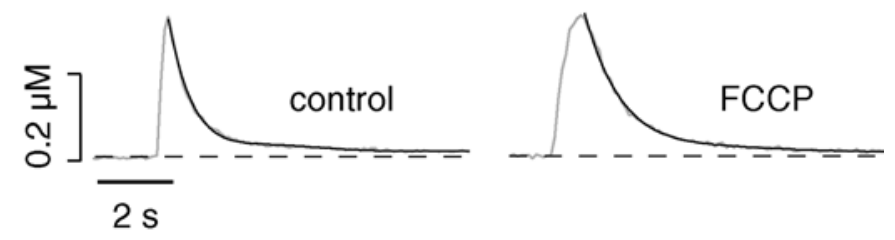

$B$

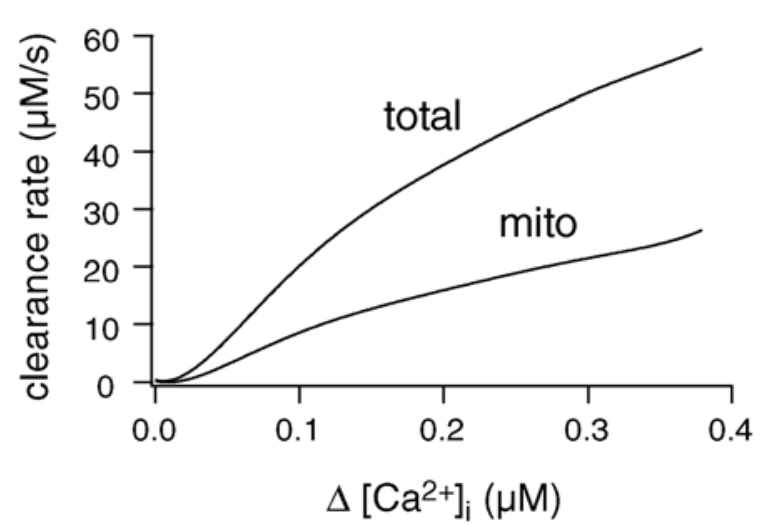

Fig. 3.1.9: Quantification of total and mitochondrial $\mathrm{Ca}^{2+}$ clearance rates using polynomial fitting. $\boldsymbol{A}$ Ratiometric recording of $\left[\mathrm{Ca}^{2+}\right]_{\mathrm{i}}$ from a patch-clamped hypoglossal MN (indicator dye $100 \mu \mathrm{M}$ fura-2) before and after addition of $1 \mu \mathrm{M}$ FCCP. $\mathrm{Ca}^{2+}$ transients were evoked by depolarisation to $0 \mathrm{mV}$. The decay of $\mathrm{Ca}^{2+}$ transients is fitted with a polynomial function of $12^{\text {th }}$ order. $\boldsymbol{B}$ The clearance rates obtained from the polynomial fit (average of 3 different cells) were corrected for $\mathrm{Ca}^{2+}$ buffering by cytosol and indicator dye and plotted as a function of the $\mathrm{Ca}^{2+}$ transient amplitude. The rate obtained from control depolarisations is a measure of the total $\mathrm{Ca}^{2+}$ clearance. The mitochondrial clearance rate was obtained by subtraction of the rate for the FCCP condition. Resting $\left[\mathrm{Ca}^{2+}\right]_{\mathrm{i}}$ was $\sim 100 \mathrm{nM}$. 


\subsubsection{Evaluation of mitochondrial activity and distribution}

The high fraction of mitochondrial $\mathrm{Ca}^{2+}$ uptake during $\mathrm{Ca}^{2+}$ transients with amplitudes below $400 \mathrm{nM}$ seemed surprising in light of the assumption that mitochondria are considered a low affinity $\mathrm{Ca}^{2+}$ uptake system (Xu et al., 1997; Rizzuto et al., 2000). Several arguments might help to overcome this apparent contradiction, including i) a high density of mitochondria in the cytosol correlating to a high level of mitochondrial activity and ii) a close proximity of mitochondria and $\mathrm{Ca}^{2+}$ influx sites. The first option was already indicated by a recent study, which evaluated the respiratory chain activity by cytochrome oxidase histochemistry in fixed rat brainstem slices (Liu \& Wong-Riley, 2003). To confirm a high level of mitochondrial activity in hypoglossal MNs in our preparation, cytochrome oxidase histochemistry was performed using young NMRI mice (11-13 days old). $12 \mu \mathrm{m}$ sections were incubated with cytochrome c reaction buffer for 3-4 hours and the dark reaction product was imaged using a digital camera. Fig. 3.1.10 shows a brainstem slice, where a variety of brainstem nuclei appear dark due to deposition of the reaction product. The hypoglossal nucleus is clearly one of the darker nuclei indicating a comparably high level of mitochondrial activity. The higher magnification reveals that the reaction product is found in the soma of hypoglossal MNs as well as in surrounding dendrites suggesting that mitochondria are present in both compartments. These findings were in good agreement with the study from Liu \& Wong-Riley.
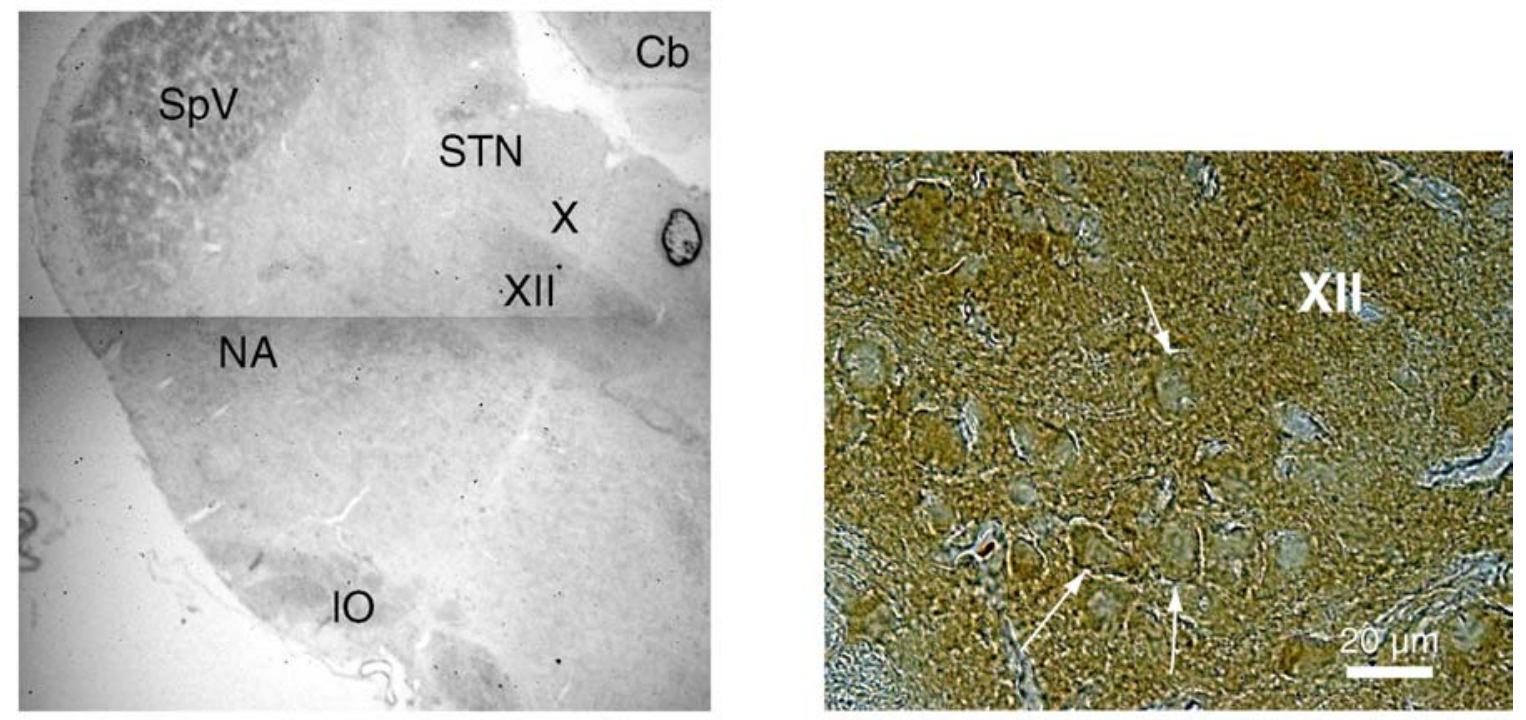

Fig. 3.1.10: Cytochrome oxidase histochemistry of fixed mouse brainstem slices. Deposition of the dark reaction product correlates with the level of mitochondrial activity. Left, $4 x$ magnification, reconstruction of two single images. Brainstem nuclei are marked as follows: hypoglossal nucleus (XII), dorsal vagal nucleus (X), solitary tract nucleus (STN), spinal trigeminal nucleus (SpV), ambiguus nucleus (NA), inferior olive (IO). Right, the hypoglossal nucleus in 40x magnification. The arrows mark motoneuron somata. The dark reaction product is found in somata and dendrites. 
To demonstrate a high level of mitochondrial activity also in the preparation that had been employed for physiological measurements, acute slices from neonatal mice were stained with $10 \mu \mathrm{g} / \mathrm{ml}$ rhodamine 123 (rhod123), which accumulates in respiring mitochondria, where its fluorescence is quenched (Fig. 3.1.11 A). After a wash-out period, mitochondria were depolarised with $1 \mu \mathrm{M}$ carbonyl cyanide 3chlorophenylhydrazone (CCCP) releasing rhod123 into the cytosol (Fig. 3.1.11 B). The intensity of rhod123 fluorescence after mitochondrial depolarisation should give an estimate of the amount of rhod123 that had been taken up previously. As shown in Fig. 3.1.11 C, the hypoglossal nucleus was characterized by a bright rhod123 fluorescence after CCCP incubation as compared to the surrounding areas. This indicated a high level of mitochondrial activity of hypoglossal MNs also in the acute slice preparation.

$\boldsymbol{A}$

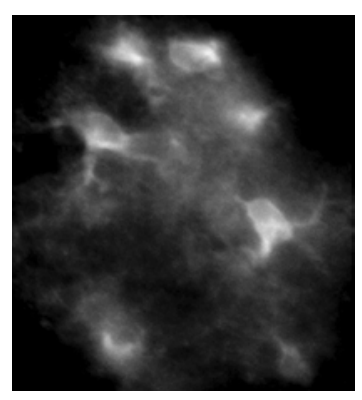

B

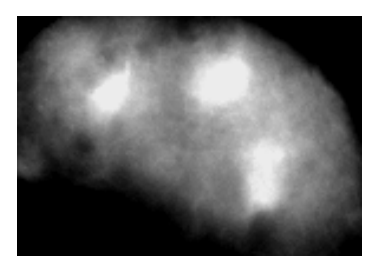

C

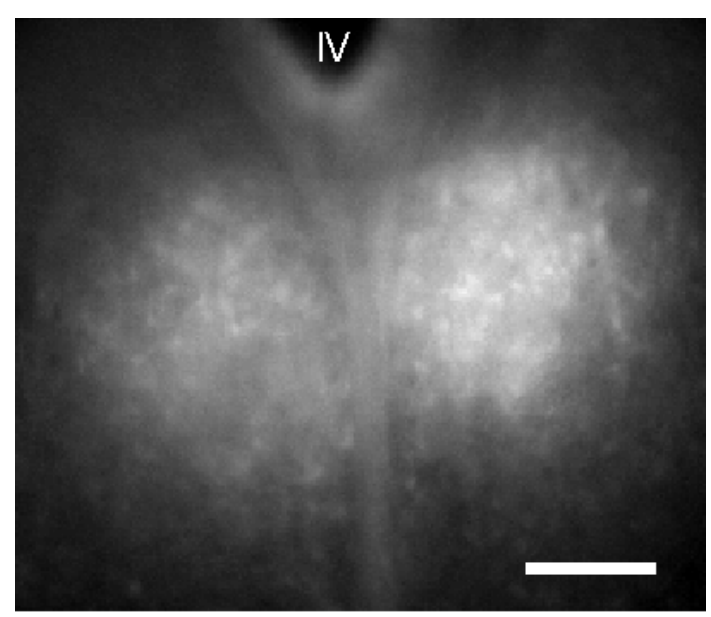

Fig. 3.1.11: CCD camera images of rhodamine 123 (rhod123) stained acute mouse brainstem slices. $\boldsymbol{A}$ Rhod123 stained cells in the hypoglossal nucleus, where the dye is mainly trapped within the mitochondria. B After incubation with $1 \mu \mathrm{M} \mathrm{CCCP}$, rhod123 is released into the cytosol. C The hypoglossal nucleus (paramedian below the IVth ventricle) is characterized by a bright rhod123 fluorescence indicating that mitochondria had taken up a considerable amount of rhod123 before depolarisation. Scale bar: $100 \mu \mathrm{m}$.

Finally, to obtain more information about the distribution of mitochondria within MNs, acute slices were stained with the mitochondrial-specific dye mitotracker green and the fluorescence pattern was imaged using confocal laser scanning microscopy. As shown in Fig. 3.1.12, the mitochondrial network extends from the soma into the 
dendrites. Although the spatial resolution limits statements about the localisation of mitochondrial clusters to the $\mu \mathrm{m}$ range, it appears that part of the mitochondria are found in close proximity to the cell membrane.
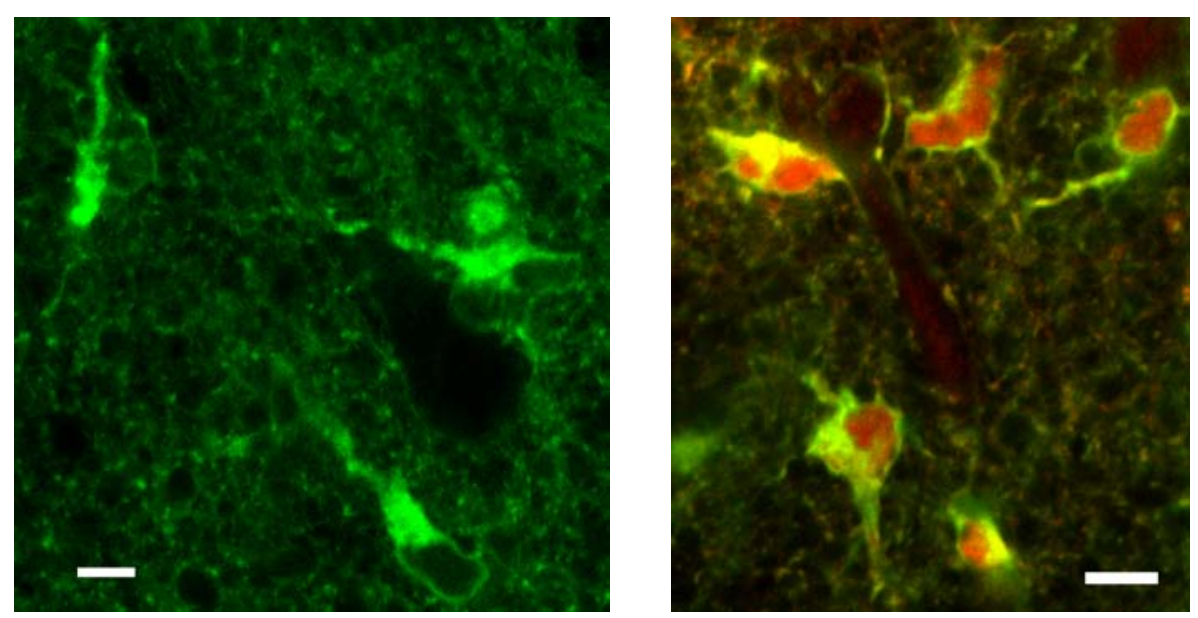

Fig. 3.1.12: Confocal laser scan images of hypoglossal MNs within acute mouse brain slices. Mitochondria are labelled with mitotracker green (green). On the right, cytosol and nucleus are additionally stained with fura-red (red). Scale bars: $10 \mu \mathrm{m}$. 


\subsection{Impact of mitochondrial dysfunction (CN) on motoneuron membrane properties}

In the following sections (3.2 and 3.3), the functional impact of an impaired mitochondrial metabolism on electrical properties and intracellular $\mathrm{Ca}^{2+}$ homeostasis of hypoglossal motoneurons is described. Mitochondrial function was disturbed by bath application of 1-2 mM sodium cyanide (CN), which inhibits complex IV of the mitochondrial electron transport chain. This protocol was chosen for several reasons, i) in ALS, a notable decrease in complex IV activity has been observed (Menzies et al., 2002a; Wiedemann et al., 2002), ii) it is considered to be a valid model for hypoxia ("chemical hypoxia"). Additionally, CN action has been described as quick and reversible (Nowicky \& Duchen, 1998; Kawai et al., 1999; Müller et al., 2002).

\subsubsection{Impact of $\mathrm{CN}$ on rhythmic motor activity}

First, the impact of $\mathrm{CN}$ on hypoglossal motoneurons integrated in a functionally intact network was investigated. For this purpose, rhythmic motor activity was recorded from hypoglossal rootlets in the thick slice preparation (see Methods section 2.3) and CN was added.

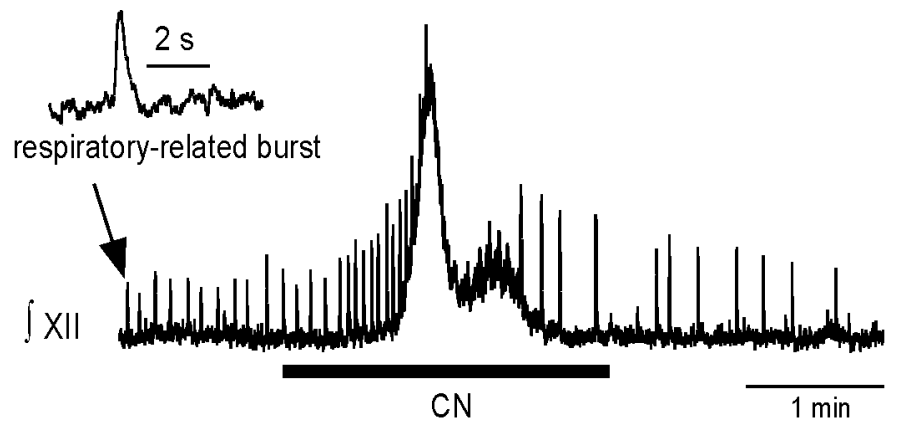

Fig. 3.2.1: Integrated recording of rhythmic respiratory-related activity from hypoglossal (XII) rootlets. Bath application of cyanide $(\mathrm{CN}, 1 \mathrm{mM})$ increases frequency and amplitude of rhythmic bursts and enhances tonic activity, indicating increased activity of hypoglossal motoneurons during $\mathrm{CN}$ treatment.

Fig. 3.2.1 shows integrated rhythmic activity recorded from hypoglossal (XII) rootlets. Within $1 \mathrm{~min}$, bath application of $1 \mathrm{mM} \mathrm{CN}$ increased the frequency of rhythmic bursts and increased the burst amplitude to $2.3 \pm 0.3$ control $(n=5)$. In the majority of cases (4 out of 5), this was followed by a transient tonic activation of the XII nerve seen as an upward shift in the baseline of the integrated XII trace, which lasted for 1-2 min. Whereas rhythmic activity characteristically turned into a frequency depression during $\mathrm{CN}$ application, burst amplitudes remained elevated during $\mathrm{CN}$ action. Wash-out of 
$\mathrm{CN}$ resulted in normalization of burst amplitudes and slow (5-10 $\mathrm{min}$ ) recovery of burst frequency. Because it is generally agreed that the amplitude of rhythmic bursts reflects the activity of hypoglossal MNs, these findings suggest that hypoglossal MNs respond to $\mathrm{CN}$ with a substantial increase in activity. It is interesting to note that the respiratory network response described here shares the same characteristics as described for deprivation of oxygen (Ramirez et al., 1997).

\subsubsection{Impact of $\mathrm{CN}$ on electrical properties of hypoglossal MNs}

After having defined the impact of impaired mitochondrial metabolism on MN network activity, $\mathrm{CN}$-induced changes were investigated on the cellular level. For this purpose, whole cell patch-clamp recordings on hypoglossal MNs in the brain slice were performed. In current clamp mode, hypoglossal MNs displayed a resting membrane potential $\left(\mathrm{V}_{\mathrm{m}}\right)$ of $-62.1 \pm 1 \mathrm{mV}(\mathrm{n}=12)$. Approximately $30 \%$ of the cells showed spontaneous spike discharge with a mean discharge frequency assessed within 1 minute before drug application of $0.27 \pm 0.19 \mathrm{~Hz}$. Upon addition of $2 \mathrm{mM} \mathrm{CN}$, hypoglossal MNs depolarised by $10.2 \pm 1.1 \mathrm{mV}(\mathrm{n}=9)$ within $15 \mathrm{~s}$ (Fig. 3.2.2 A) and mean discharge frequencies increased to $0.46 \pm 0.2 \mathrm{~Hz}(p<0.01)$. CN also increased synaptic activity as exemplified i.e. in Fig. 3.3.1 A. After washout of $\mathrm{CN}$, potential changes reversed within 1 minute, whereas synaptic and action potential activity returned to baseline levels within 3 minutes.

To investigate the relative contribution of synaptic activity and firing rates, CN applications were performed in the presence of synaptic blockers (10 $\mu \mathrm{M} \mathrm{CNQX,100}$ $\mu \mathrm{M}$ APV, $10 \mu \mathrm{M}$ bicuculline and $10 \mu \mathrm{M}$ strychnine) and/or TTX (Fig. 3.2.2 B, C). When postsynaptic receptors were blocked, hypoglossal MNs displayed a mean resting $V_{m}$ of $-63.3 \pm 1.6 \mathrm{mV}(\mathrm{n}=12)$ and a $\mathrm{CN}$-induced depolarisation of $13.8 \pm 3 \mathrm{mV}$ $(n=11)$, which was comparable to control responses $(p>0.05)$. When $0.5 \mu$ M TTX was added alone or in addition to synaptic blockers, $\mathrm{CN}$-induced depolarisations were again comparable to control (11.3 $\pm 1.7 \mathrm{mV}, \mathrm{n}=7 ; 13.3 \pm 2 \mathrm{mV}, \mathrm{n}=6 ; \mathrm{p}>0.05)$, indicating that they primarily resulted from intrinsic membrane conductances. 
Results

$A$

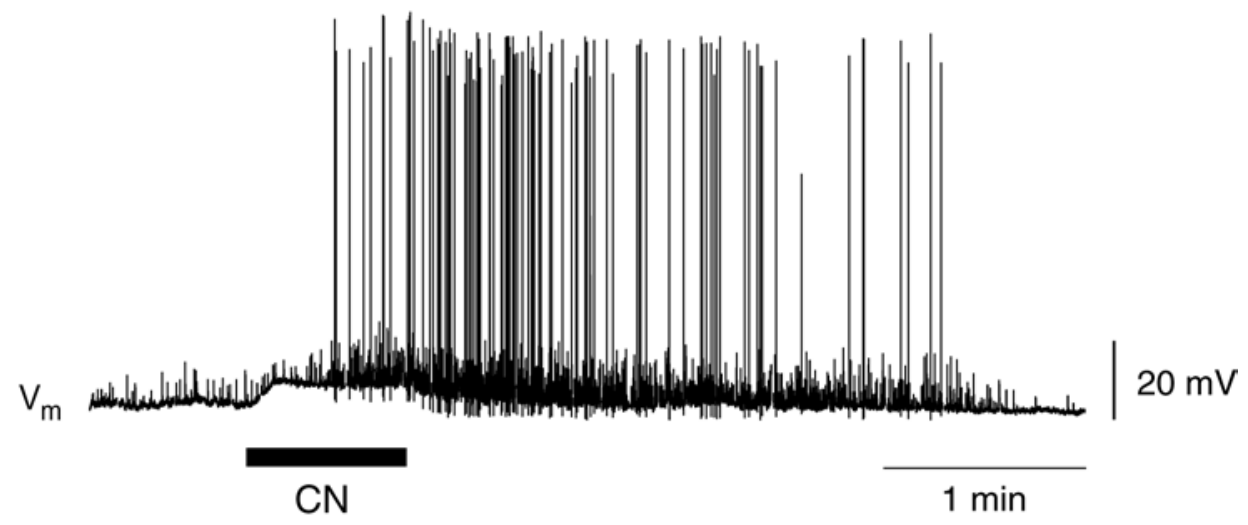

$B$

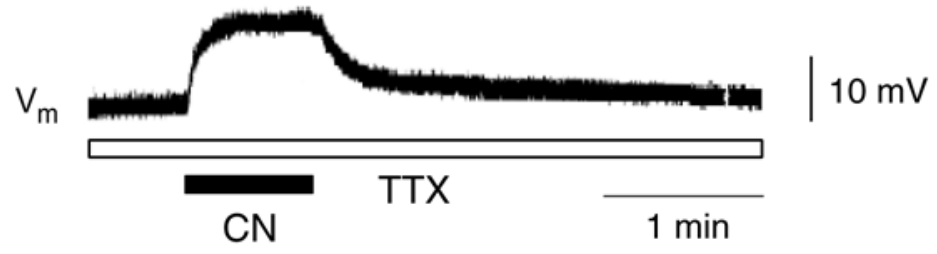

C

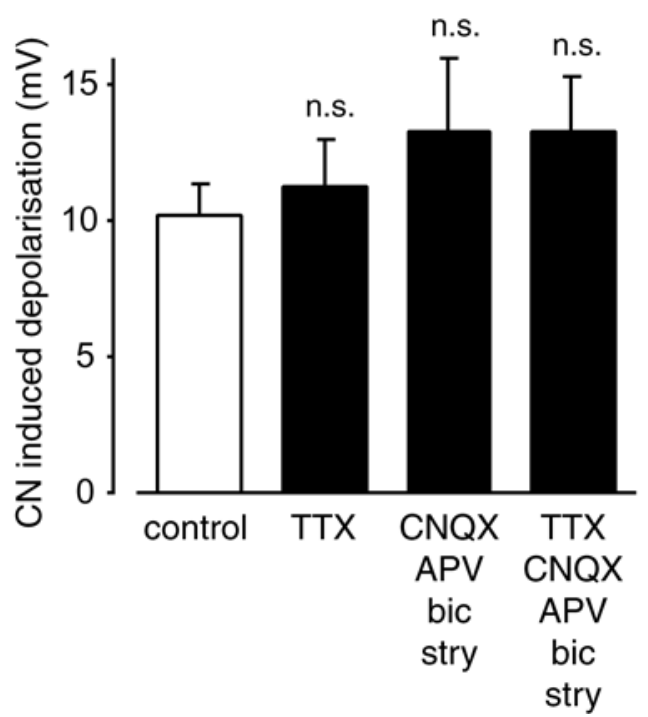

Fig. 3.2.2: $\boldsymbol{A}$ Membrane potential $\left(\mathrm{V}_{\mathrm{m}}\right)$ recorded from a patch-clamped hypoglossal $M N$ in acute mouse brainstem slice. Bath application of $\mathrm{CN}(2 \mathrm{mM})$ quickly induces $\mathrm{MN}$ depolarisation and enhances synaptic activity, leading to spontaneous action potential generation. Wash out of $\mathrm{CN}$ repolarises membrane potential and reduces synaptic activity over 1-2 minutes, finally action potential activity stops. $\boldsymbol{B}, \boldsymbol{C}$ The depolarising response of hypoglossal MNs to $\mathrm{CN}$ persists in the presence of TTX $(0.5 \mu \mathrm{M})$ and/or during blockade of postsynaptic receptors with (in $\mu \mathrm{M}) 10 \mathrm{CNQX}, 100 \mathrm{APV}, 10$ bicuculline and 10 strychnine, indicating that membrane depolarisation was mediated by changes in MN membrane properties and not by changes in synaptic transmission or glutamate release. 


\subsubsection{Differential response of vulnerable and resistant neurons to $\mathrm{CN}$}

In order to test whether the increased neuronal excitability of hypoglossal MNs during $\mathrm{CN}$ action was a feature attributable to MNs or if this might represent a general cellular response in our preparation, we performed complementary recordings from vulnerable facial (VII) MNs and neurons of the nucleus dorsalis vagus $(X)$, which is located directly dorsal of the hypoglossal nucleus and which is typically tolerant to hypoxia and resistant to damage in ALS.

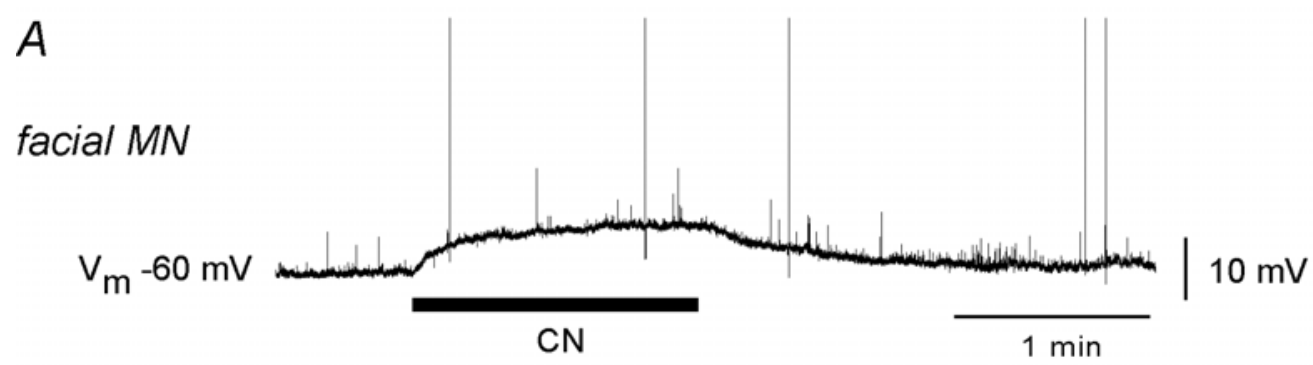

$B$

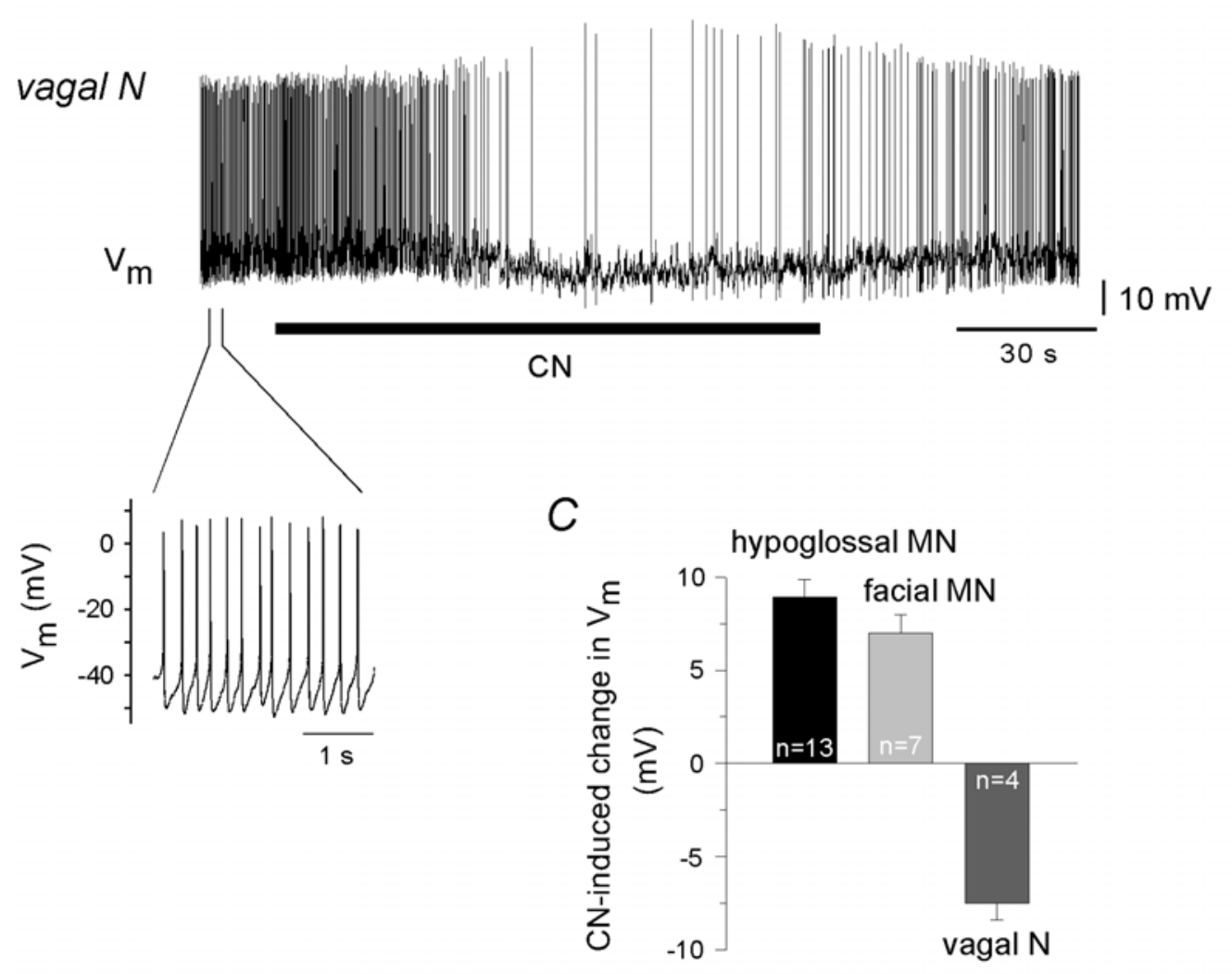

Fig. 3.2.3: $\boldsymbol{A}$ Membrane potential $\left(\mathrm{V}_{\mathrm{m}}\right)$ recorded from a patch-clamped $\mathrm{MN}$ in the facial nucleus (VII). Bath application of CN $(2 \mathrm{mM})$ depolarises facial MNs by $7 \pm 1 \mathrm{mV}(\mathrm{n}=7)$ with repolarisation after wash-out of $C N$. $B \vee_{m}$ recorded from a patch-clamped neuron in the nucleus dorsalis vagus $(X)$. Addition of CN (2 mM) reversibly hyperpolarizes the vagal neuron by $7.5 \pm 0.9 \mathrm{mV}(\mathrm{n}=4)$ and reduces spontaneous spike discharge. C Comparison of the $\mathrm{CN}$-induced $\mathrm{V}_{\mathrm{m}}$ change in vulnerable $\mathrm{MNs}$ (hypoglossal and facial) and resistant vagal neurons. (Intracelluar solution contained $100 \mu \mathrm{M}$ fura-2.) 
In current clamp mode, facial MNs displayed a resting $\mathrm{V}_{\mathrm{m}}$ of $-61.7 \pm 1.7 \mathrm{mV}(\mathrm{n}=7)$. Similar to hypoglossal MNs, addition of CN reversibly depolarised facial MNs by $7 \pm 1$ $\mathrm{mV}(\mathrm{n}=7$; Fig. 3.2.3 A). In contrast, vagal neurons showed a resting membrane potential around $-41 \mathrm{mV}$ and displayed tonic spike discharge at frequencies of 3-4 $\mathrm{Hz}$ (Fig. 3.2.3 B). CN hyperpolarized vagal neurons by $7.5 \pm 0.9 \mathrm{mV}(\mathrm{n}=4)$ and reduced action potential firing, which was consistent with previous studies showing that $\mathrm{CN}$ activates hyperpolarizing ATP-sensitive $\mathrm{K}^{+}$channels (Kulik et al., 2002; Müller et al., 2002). Taken together, these experiments indicated that the $\mathrm{CN}$-induced increase in electrical excitability was specific for vulnerable MNs.

\subsubsection{CN-induced inward currents $\left(I_{\mathrm{CN}}\right)$}

Membrane conductances in hypoglossal MNs were further investigated in voltage clamp mode, where bath application of $2 \mathrm{mM} \mathrm{CN}$ activated an inward current $\mathrm{I}_{\mathrm{CN}}=$ $-51 \pm 9 \mathrm{pA}(\mathrm{n}=8$, holding potential $-60 \mathrm{mV})$. Its magnitude was relatively constant in a voltage range of -80 to $-40 \mathrm{mV}$ as revealed by voltage ramp protocols $(20 \mathrm{mV} / 100$ ms, not shown). To identify the underlying charge carrier, $I_{\mathrm{CN}}$ was investigated under different ionic and pharmacological conditions (Fig. 3.2.4). At first, the potential contribution of persistent, TTX-sensitive $\mathrm{Na}^{+}$channels was studied, which have recently been described in hypoglossal MNs (Powers \& Binder, 2003). In agreement with the current clamp data (compare Fig. 3.2.2), TTX did not significantly alter $\mathrm{I}_{\mathrm{CN}}$ $(-56 \pm 13 \mathrm{pA}, \mathrm{n}=7, \mathrm{p}>0.05)$. A contribution of $\mathrm{K}^{+}$conductances was probed by blocking $\mathrm{K}^{+}$currents with tetraethylammonium chloride (TEA, $20 \mathrm{mM}$ internal and 10 $\mathrm{mM}$ external) and replacing $\mathrm{K}^{+}$by caesium in the pipette, without any significant effect $\left(I_{C N}=-62 \pm 15 p A, n=10, p>0.05\right)$. Blockade of $\mathrm{Ca}^{2+}$ currents by the nonselective $\mathrm{Ca}^{2+}$ channel blocker $\mathrm{CdCl}_{2}(200 \mu \mathrm{M})$ also did not significantly change $\mathrm{I}_{\mathrm{CN}}$ $(-46 \pm 8 \mathrm{pA}, \mathrm{n}=4, \mathrm{p}>0.05)$. The observation that $\mathrm{Ca}^{2+}$ was not the main charge carrier was additionally confirmed by experiments in current clamp mode, where removal of $\mathrm{Ca}^{2+}$ from the extracellular solution $\left(\mathrm{Ca}^{2+}\right.$ free aCSF containing $\left.1 \mathrm{mM} \mathrm{EGTA;} \mathrm{n}=3\right)$ did not influence $\mathrm{CN}$-induced depolarisations (Fig. 3.2.4 B). 
Results

$A$

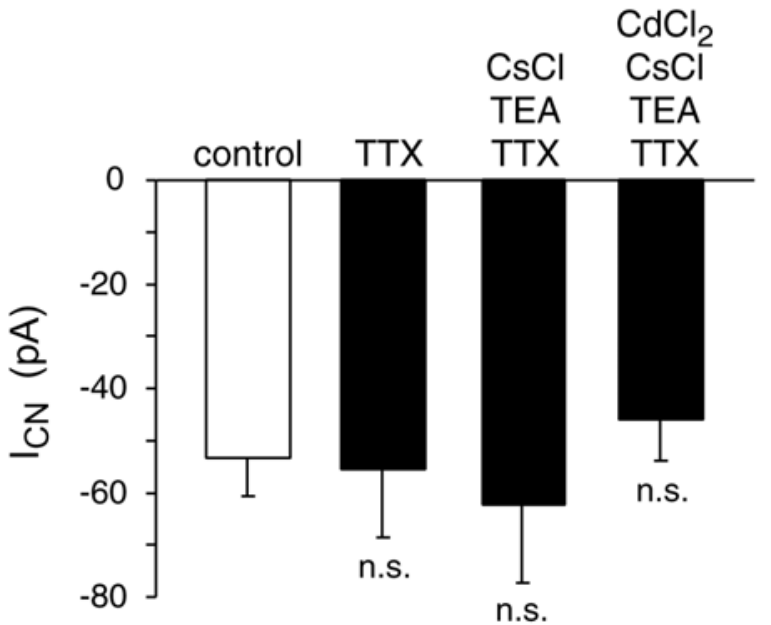

C

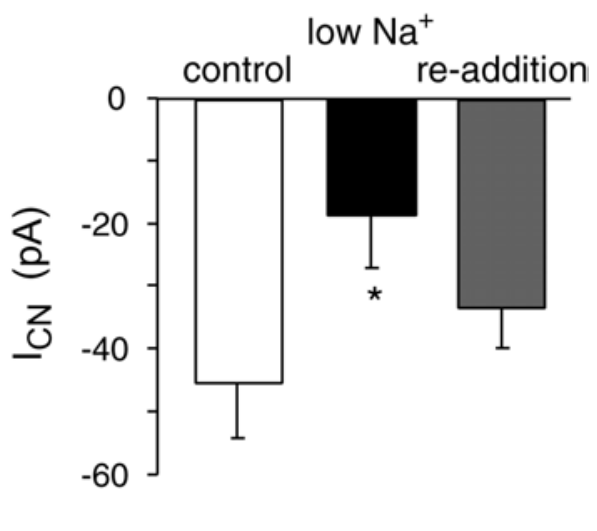

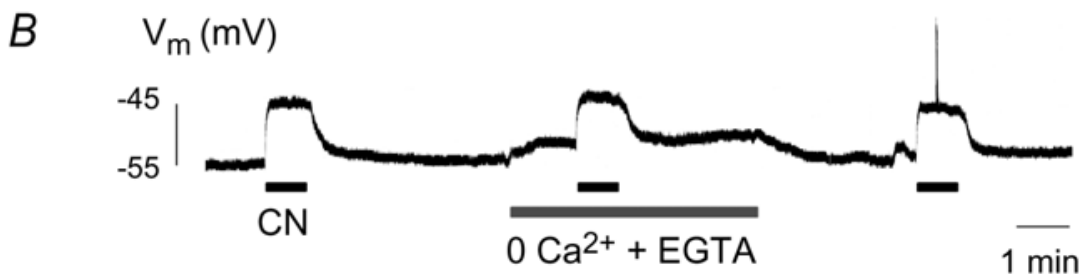

Fig. 3.2.4: The magnitude of inward current $\left(\mathrm{I}_{\mathrm{CN}}\right.$, voltage clamp, HP -60 or $\left.-70 \mathrm{mV}\right)$ or membrane depolarisation (current clamp) induced by $2 \mathrm{mM} \mathrm{CN}$ is compared under different experimental conditions. $\boldsymbol{A}$ No significant difference in the amplitude of $\mathrm{I}_{\mathrm{CN}}$ is found when TTX is present in the aCSF compared to the control condition (control: $-51 \pm 9$ pA, $n=8$; TTX: $-56 \pm 13$ pA, n=7). Also blockade of potassium currents with TEA and replacement of intracellular potassium by caesium does not significantly alter $\mathrm{I}_{\mathrm{CN}}$ as compared to the control condition (TTX, TEA, CsCl: $-62 \pm 15 \mathrm{pA}, \mathrm{n}=10$ ), nor did additional wash-in of cadmium to block $\mathrm{Ca}^{2+}$ conductances (TTX, TEA, $\mathrm{CsCl}, \mathrm{CdCl}_{2}$ : $-46 \pm 8$ $\mathrm{pA}, \mathrm{n}=4)$. $\boldsymbol{B} \mathrm{CN}$ depolarises $\mathrm{V}_{\mathrm{m}}$ even when $\mathrm{Ca}^{2+}$ is completely removed from the extracellular solution (containing $1 \mathrm{mM}$ EGTA), further indicating that $\mathrm{Ca}^{2+}$ is not the charge carrier of $\mathrm{I}_{\mathrm{CN}}$. $\boldsymbol{C}$ Reducing the extracellular $\mathrm{Na}^{+}$concentration from 144 to $26 \mathrm{mM}$ decreases $I_{\mathrm{CN}}$ to $40 \pm 13 \%$ of the control value $\left(\mathrm{I}_{\mathrm{CN}}\right.$ control: $45 \pm 9 \mathrm{pA}$, low $\left.\mathrm{Na}^{+}: 18 \pm 9, \mathrm{p}<0.05, \mathrm{n}=4\right)$, re-addition of $\mathrm{Na}^{+}$increased $\mathrm{I}_{\mathrm{CN}}$ back to $76 \pm 10 \%$, indicating that $\mathrm{Na}^{+}$is the main charge carrier of $\mathrm{I}_{\mathrm{CN}}$ at the given membrane potential. ( $A-B$, intracellular solution contained $100 \mu \mathrm{M}$ fura-2)

Interestingly, $\mathrm{I}_{\mathrm{CN}}$ reversed around $50 \mathrm{mV}$ after blockade of $\mathrm{K}^{+}, \mathrm{Ca}^{2+}$ and TTXsensitive $\mathrm{Na}^{+}$channels. Since the reversal potential for chloride $\left(\mathrm{Cl}^{-}\right)$was chosen $\sim 0 \mathrm{mV}$, TTX-insensitive $\mathrm{Na}^{+}$conductances were identified as a potential charge carrier. To evaluate this, we changed the extracellular $\mathrm{Na}^{+}$concentration from 144 $\mathrm{mM}$ to $26 \mathrm{mM}$ (replacement of $\mathrm{NaCl}$ by choline chloride), which reduced $\mathrm{I}_{\mathrm{CN}}$ to $40 \pm$ $13 \%$ of control ( $I_{\mathrm{CN}}$ control: $45 \pm 9$ pA, low Na+: $18 \pm 9$ pA, p<0.05, n=4; Fig. 3.2.4 C). Re-addition of $\mathrm{Na}^{+}$to the perfusion solution substantially increased $\mathrm{I}_{\mathrm{CN}}(76 \pm 10 \%$ of control). Taken together, these experiments identify TTX-insensitive $\mathrm{Na}^{+}$ conductances as prominent charge carriers of $\mathrm{I}_{\mathrm{CN}}$ in hypoglossal motoneurons. 
Coming back to the $\mathrm{CN}$-induced change in membrane conductance in vagal neurons, it was possible that the pronounced activation of $\mathrm{K}^{+}$conductances in these cells covered an activation of smaller inward currents. Therefore, in vagal neurons, a potential activation of inward currents by $\mathrm{CN}$ was tested, when $\mathrm{K}^{+}$was replaced by caesium in the pipette solution and $\mathrm{K}^{+}$channels were additionally blocked with 10 $\mathrm{mM}$ TEA in the bath solution. Cells were held between -40 and $-50 \mathrm{mV}$ and $2 \mathrm{mM}$ $\mathrm{CN}$ was applied for 1-3 minutes. This hardly affected membrane currents (maximum observed inward current: $6 \mathrm{pA}$, not shown) indicating that vagal neurons do not posses $\mathrm{CN}$-sensitive inward currents.

\subsubsection{Activation profile of $I_{\mathrm{CN}}$}

An interesting question was related to the cellular mechanisms that mediated the fast onset of $\mathrm{I}_{\mathrm{CN}}$. As the primary cellular target of $\mathrm{CN}$ is the mitochondrial respiratory chain, CN application is expected to block complex IV and collapse the potential gradient $(\Delta \psi)$ across the inner mitochondrial membrane. Inhibition of complex IV is further thought to increase the concentrations of physiological electron donors NADH and $\mathrm{FADH}_{2}$.

\section{$\underline{I_{C N} \text { in relation to NADH and mitochondrial potential }(\Delta \psi)}$}

The intrinsic fluorescence of NADH allows mitochondrial metabolism to be indirectly monitored in the slice without addition of fluorescent dyes. In experiments performed at the Dept. of Applied \& Engineering Physics at Cornell University, two-photon excitation was used to characterize the NADH fluorescence in the hypoglossal nucleus. The hypoglossal nucleus was easily recognized by a high level of intrinsic fluorescence in both motoneurons and astrocytes (Fig. 3.2.5 A). To prove NADH fluorescence as an indicator of mitochondrial metabolism, control experiments were performed, in which respiratory chain activity was inhibited by hypoxia (Fig. 3.2.5 B) or CN (1 mM; Fig. 3.2.5 C). Both protocols reversibly enhanced the intrinsic NADH fluorescence in both neurons and astrocytes. This observation confirmed the use of $\mathrm{NADH}$ imaging for assessment of mitochondrial metabolism. 

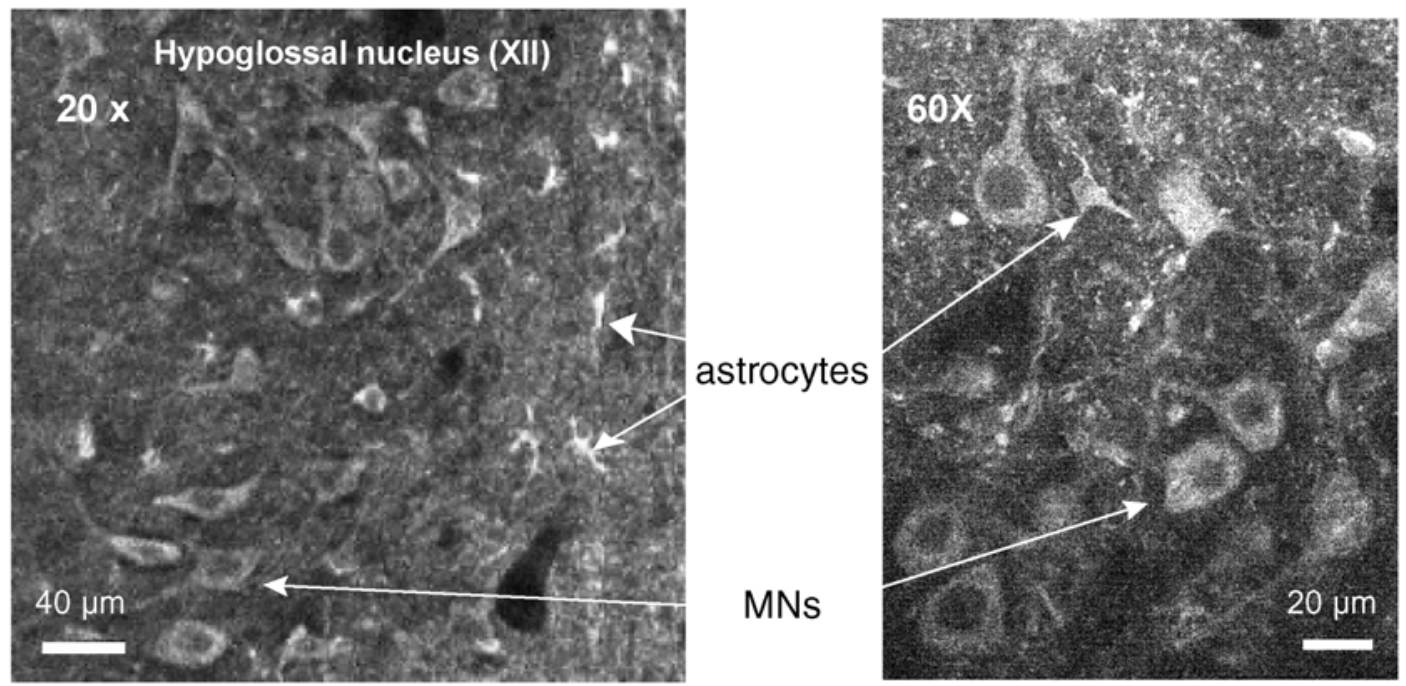

$B$

hypoxia $\left(\mathrm{N}_{2}\right)$

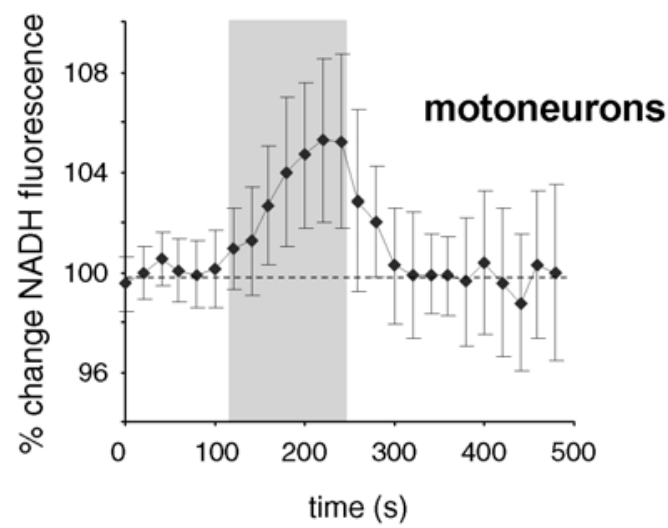

hypoxia $\left(\mathrm{N}_{2}\right)$

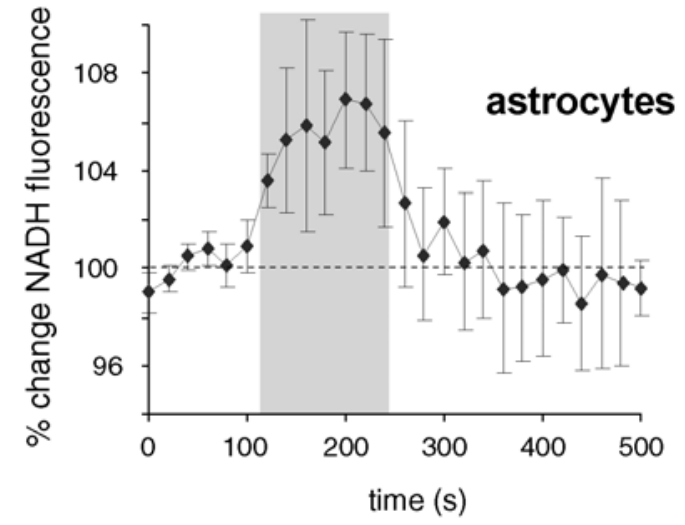

C
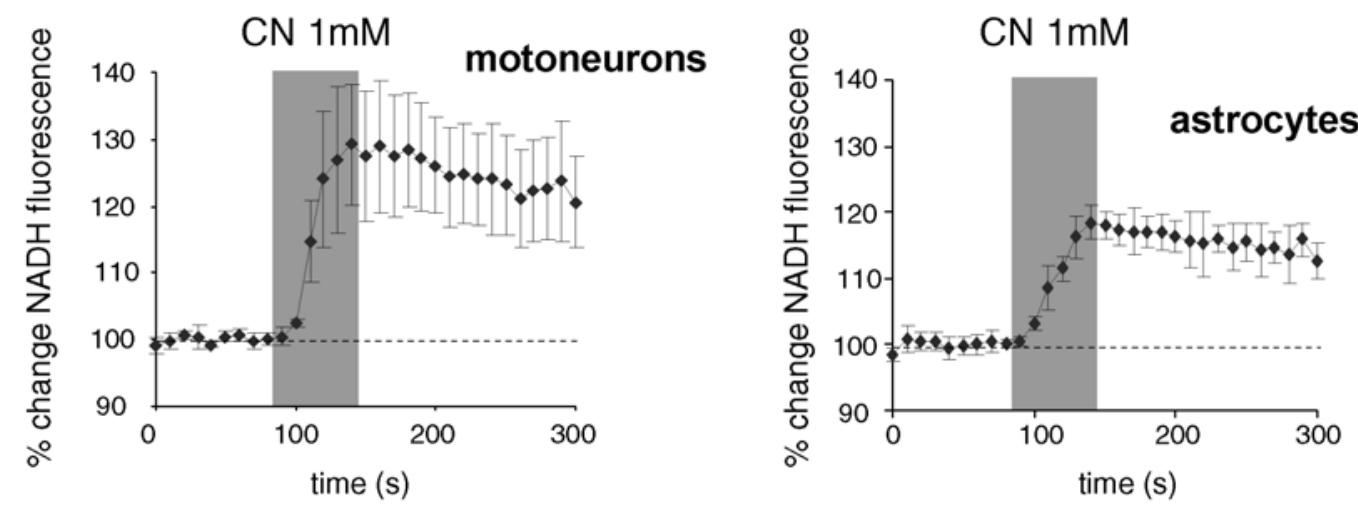

Fig. 3.2.5: Two-photon imaging of intrinsic NADH fluorescence in acute mouse brainstem slices. $\boldsymbol{A}$ The hypoglossal nucleus is characterized by a bright NADH fluorescence both in motoneurons (MNs) and astrocytes (arrows). $\boldsymbol{B}$ Inhibition of the respiratory chain by hypoxia reversibly increases NADH fluorescence both in motoneurons $(n=12)$ and astrocytes $(n=3)$. C A similar effect is observed during mitochondrial inhibition with $1 \mathrm{mM} \mathrm{CN}(8 \mathrm{MNs}, 5$ astrocytes). 
In further experiments performed in Göttingen, the $\mathrm{CN}$-induced changes in NADH were monitored in parallel to changes in $\Delta \psi$ and $V_{m}$ in patch-clamped hypoglossal MNs by using CCD camera imaging (rhod123 as indicator of $\Delta \psi$; Fig. 3.2.6 A). Addition of $2 \mathrm{mM} \mathrm{CN}$ to the bathing solution increased rhod123 fluorescence as well as NADH autofluorescence with a delay after onset of depolarisation of $3 \pm 2.2 \mathrm{~s}$ (rhod123, n=6) and $6.3 \pm 1.5 \mathrm{~s}(\mathrm{NADH}, \mathrm{n}=8)$. To test whether the onset of $\mathrm{I}_{\mathrm{CN}}$ was dependent on depolarisation of $\Delta \psi$, the mitochondrial uncoupler p-trifluoromethoxyphenylhydrazone (FCCP; $1 \mu \mathrm{M})$ was added. FCCP shunts the proton gradient over the inner mitochondrial membrane, thus depolarising mitochondria while respiratory chain activity continues. As expected, FCCP increased rhod123 fluorescence, but left $\mathrm{NADH}$-autofluorescence essentially unaffected. FCCP also failed to induce changes in $\mathrm{V}_{\mathrm{m}}\left(\mathrm{V}_{\mathrm{m}}\right.$ change during FCCP: $1 \pm 2 \mathrm{mV}, \mathrm{n}=6, \mathrm{p}<0.01$, Fig. 3.2.6 B) and did not induce inward currents in voltage clamp mode $(-60 \mathrm{mV} ; \mathrm{n}=3)$, indicating that activation of $\mathrm{I}_{\mathrm{CN}}$ was independent of $\Delta \psi$ depolarisation.

$A$

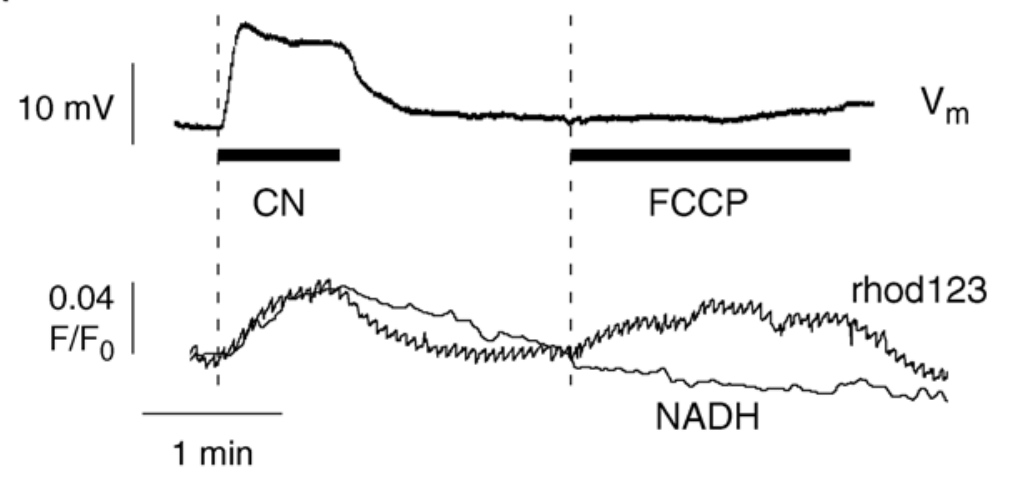

$B$

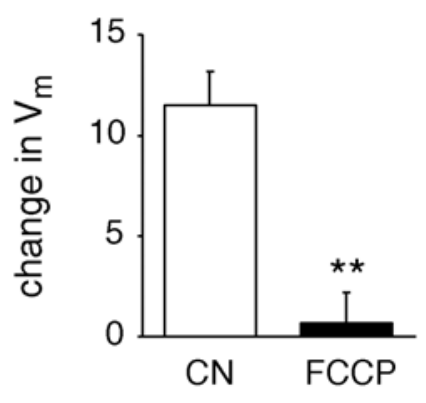

Fig. 3.2.6: $\boldsymbol{A} C C D$ camera imaging of intrinsic $N A D H$ fluorescence and mitochondrial potential $(\Delta \psi)$ using rhod123 in a patch-clamped hypoglossal MN. Both CN $(2 \mathrm{mM})$ and FCCP $(1 \mu \mathrm{M})$ depolarise mitochondria, but FCCP does not increase NADH. FCCP also fails to increase $V_{m}$ indicating that $\mathrm{CN}$ induced $\mathrm{MN}$ depolarisation is independent of mitochondrial depolarisation. $\boldsymbol{B}$ Comparison of $\mathrm{CN}$ and FCCP induced changes in $\mathrm{V}_{\mathrm{m}}$.

\section{$\underline{I_{C N} \text { mimicked by azide }}$}

It was then tested whether activation of $\mathrm{I}_{\mathrm{CN}}$ could be reproduced by other substances that interfere with the mitochondrial respiratory chain. Whereas the complex I inhibitor rotenone $(25-50 \mu \mathrm{M}, \mathrm{n}=3)$ failed to alter $\mathrm{V}_{\mathrm{m}}$ within 5 minutes (Fig. 3.2.7 A), the complex IV inhibitor, sodium azide $(2 \mathrm{mM})$ depolarised the plasma membrane by 7.1 $\pm 1 \mathrm{mV}$ and increased AP discharge similarly to $\mathrm{CN}(\mathrm{n}=9$; Fig. 3.2.7 B). These 
findings suggested that the mechanism that mediated activation of $I_{C N}$ was located downstream of complex IV.

$A$

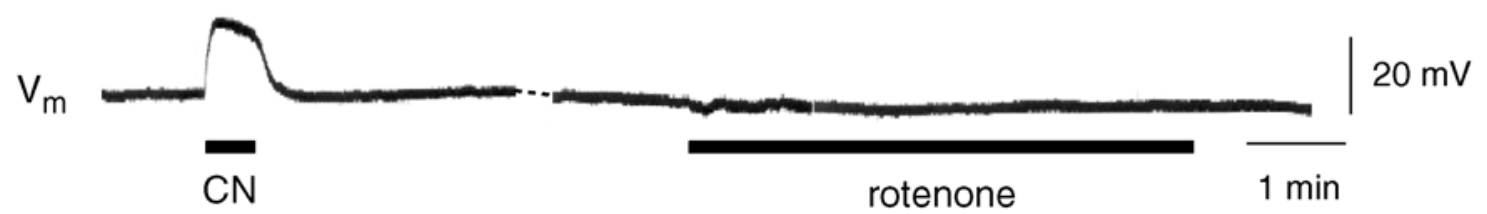

$B$

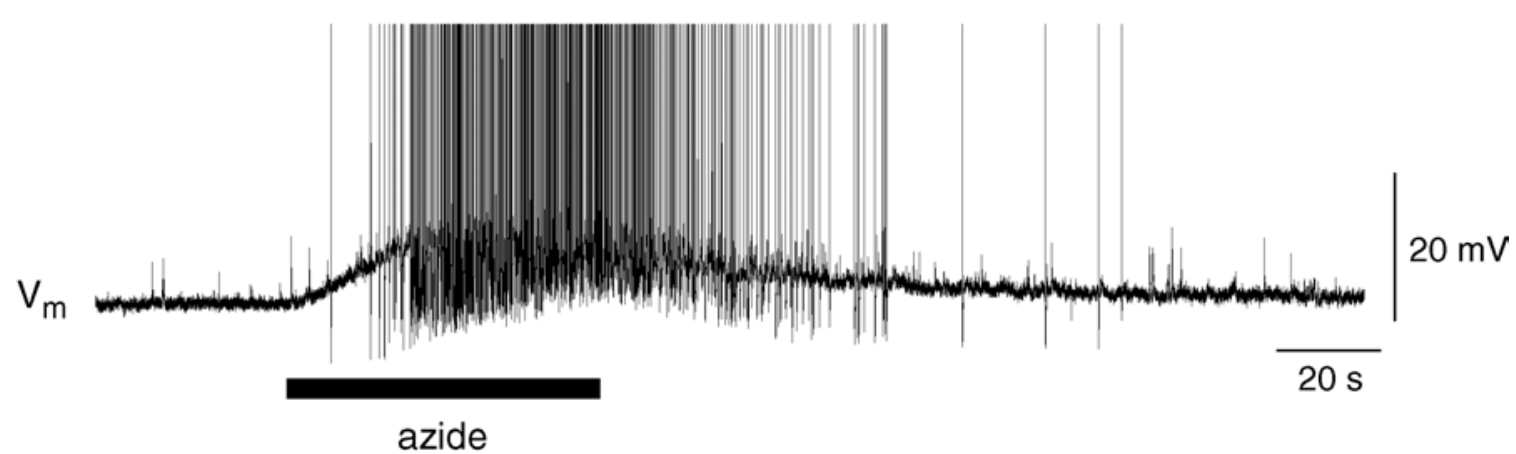

Fig. 3.2.7: Whole-cell current clamp recordings from hypoglossal MNs. $\boldsymbol{A}$, The complex I inhibitor rotenone fails to alter $\mathrm{V}_{\mathrm{m}}$, whereas $(\boldsymbol{B})$ the complex IV inhibitor sodium azide $(2 \mathrm{mM})$ mimics the $\mathrm{CN}$ induced depolarisation and increases action potential discharge (APs were truncated). In $A$, blockers of postsynaptic receptors (CNQX, AP-5, bicuculline, strychnine) were present in the aCSF.

\section{Effect of antioxidants on $\mathrm{I}_{\mathrm{CN}}$}

The next possibility considered was that inhibition of complex IV may increase the formation of superoxide $\left(\mathrm{O}_{2}{ }^{-}\right)$by transfer of electrons to oxygen at the semiubiquinone site of the respiratory chain. Superoxide and related reactive oxygen species (ROS) may act as signaling molecules by shifting redox pairs to the oxidized state (Lopez-Barneo et al., 2001). The potential involvement of ROS in mediating $I_{C N}$ was tested by the ability of the antioxidants and ROS scavengers ascorbic acid and trolox ${ }^{\circledR}$ to interfere with the induction of $I_{C_{N}}$. Brain slices were pre-incubated with 1 $\mathrm{mM}$ ascorbic acid and $750 \mu \mathrm{M}$ trolox ${ }^{\circledR}$, a derivative of $\alpha$-tocopherol (vitamin E), for 30-50 min, and the drugs were also present during the following $\mathrm{CN}$ exposure (2 mM for 50-60 s). The drug concentrations used were shown to be effective in previous studies in vitro (Vergun et al., 2001; MacGregor et al., 2003). Pre-incubation with ascorbic acid and trolox ${ }^{\circledR}$ reduced the mean amplitude of $\mathrm{I}_{\mathrm{CN}}$ to $40 \%(-22 \pm 6 \mathrm{pA}$, 
$\mathrm{n}=8$ ) of the value that had been found under control conditions $(\mathrm{p}<0.01$; Fig 3.2.8). In two of the eight cells tested, $\mathrm{I}_{\mathrm{CN}}$ was totally abolished.

Taken together, the experiments suggested that an increase in the formation of ROS following complex IV inhibition was involved in the activation of $\mathrm{I}_{\mathrm{CN}}$.

$A$
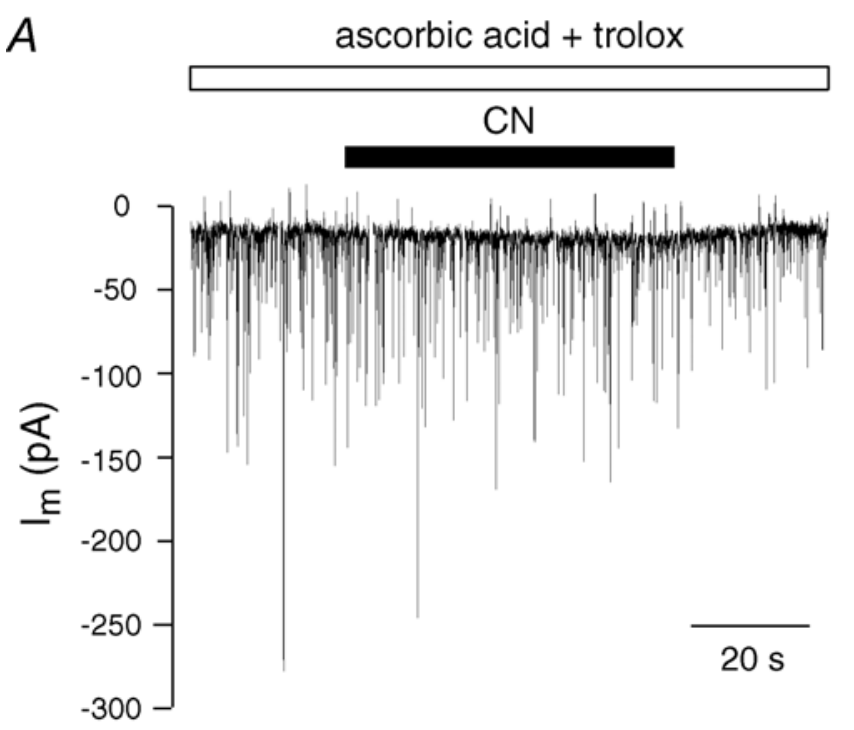

$B$

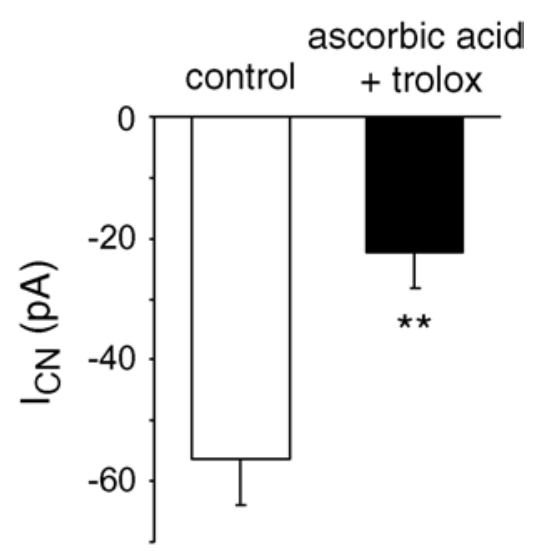

Fig. 3.2.8: $\boldsymbol{A}$ Whole-cell voltage clamp recording from a hypoglossal $\mathrm{MN}$. The antioxidants ascorbic acid $(1 \mathrm{mM})$ and trolox $(750 \mu \mathrm{M})$ prevent induction of the inward current by $\mathrm{CN}$. $\boldsymbol{B}$ In summary, ascorbic acid and trolox reduce $\mathrm{I}_{\mathrm{CN}}$ to $40 \%(-22 \pm 6 \mathrm{pA})$ of the value that was found under control conditions $(p<0.01, n=8)$, indicating that a redox mechanisms was involved in activation of $I_{C N}$, possibly due to an increased production of reactive oxygen species (ROS). 


\subsection{Impact of mitochondrial dysfunction (CN) on motoneuron $\mathrm{Ca}^{2+}$ levels}

\subsubsection{Impact of $\mathrm{CN}$ on resting $\left[\mathrm{Ca}^{2+}\right]_{\mathrm{i}}$}

To investigate the impact of impaired mitochondrial metabolism on motoneuron $\left[\mathrm{Ca}^{2+}\right]_{\mathrm{i}}$, electrophysiological recordings were combined with calcium imaging measurements. The patch-clamp attempt was justified as there was no apparent difference in mitochondrial $\mathrm{Ca}^{2+}$ handling between patch-clamped and AM-ester loaded cells, but it provided additional information about the electrical properties of hypoglossal MNs.

$A$

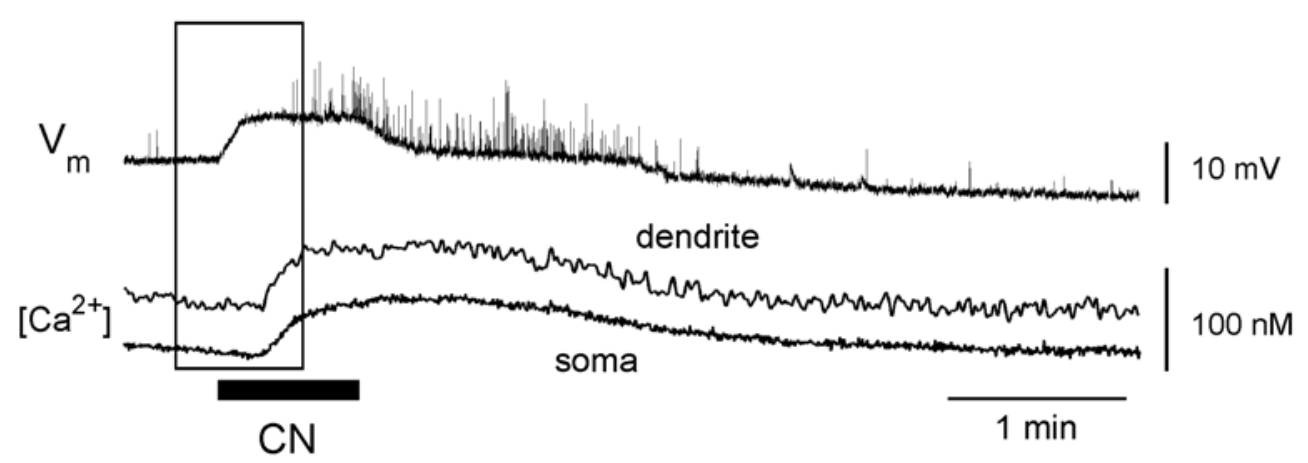

$B$

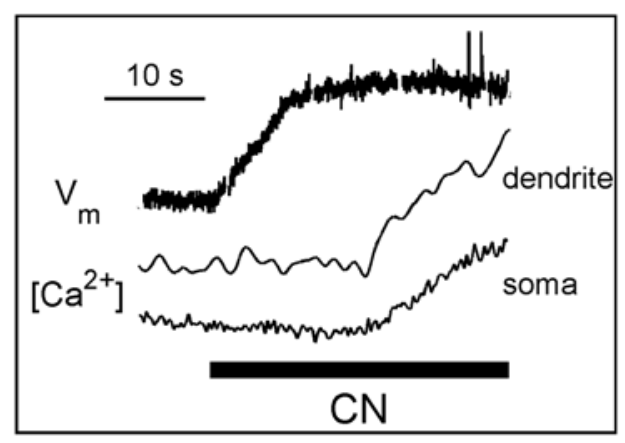

C

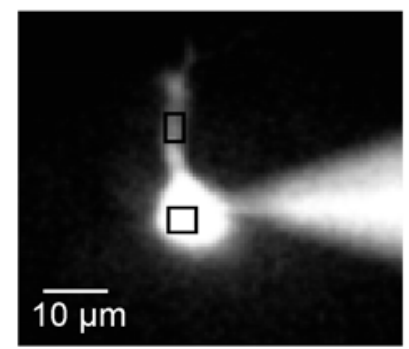

Fig 3.3.1: $\boldsymbol{A}$ Simultaneous recording of membrane potential $\left(\mathrm{V}_{\mathrm{m}}\right)$ and $\left[\mathrm{Ca}^{2+}\right]_{\mathrm{i}}$ in a patch-clamped hypoglossal MN. Action potential activity was blocked with $0.5 \mu \mathrm{M}$ TTX. Bath application of CN $(2 \mathrm{mM})$ first depolarised $V_{m}$ and then increased resting $\left[\mathrm{Ca}^{2+}\right]_{i}$ with a delay of $\sim 18 \mathrm{~s}$ both in the soma and primary dendrites $(\boldsymbol{B}, \boldsymbol{C})$. After wash-out of $\mathrm{CN},\left[\mathrm{Ca}^{2+}\right]_{i}$ slowly returned to baseline.

In current clamp, short (45-60 s) applications of $\mathrm{CN}$ increased basal $\left[\mathrm{Ca}^{2+}\right]_{\mathrm{i}}$ by two different mechanisms. First, a small increase in $\left[\mathrm{Ca}^{2+}\right]_{i}$ independent of action potential (AP) generation or synaptic activity was observed (Fig. 3.3.1 A-C). This augmentation, most likely mediated by mitochondria-controlled $\mathrm{Ca}^{2+}$ release from intracellular stores (see below), started $19 \pm 3 s(n=7)$ after wash-in of $\mathrm{CN}$ and 
displayed amplitudes ranging from 10 to $50 \mathrm{nM}$, which decreased upon repetitive $\mathrm{CN}$ exposure.

$A$

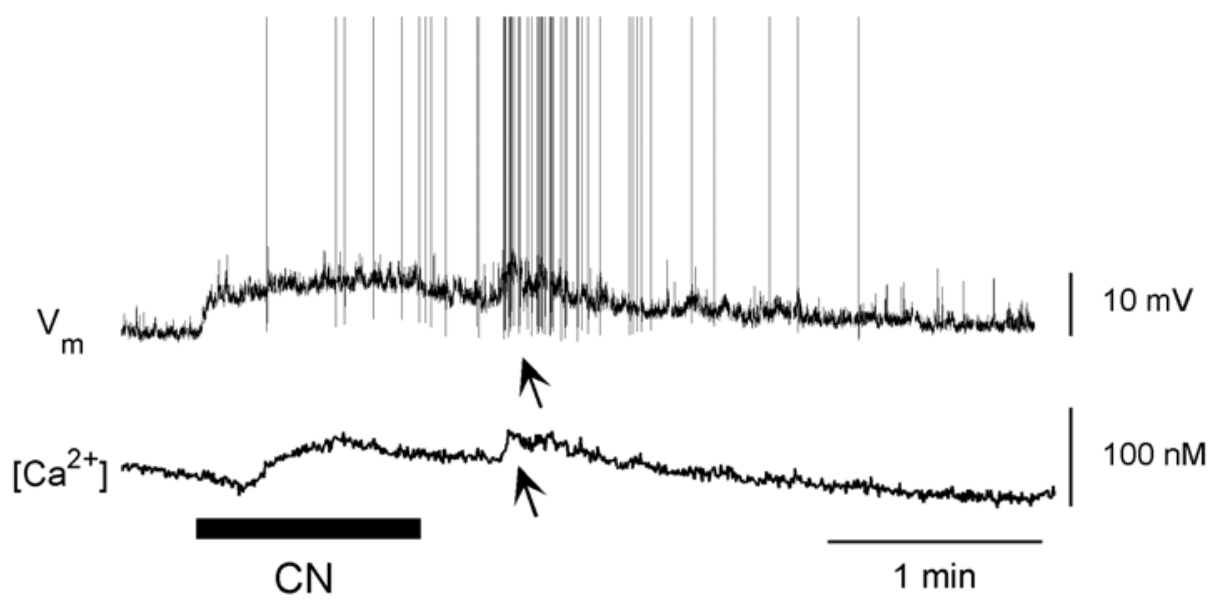

$B$
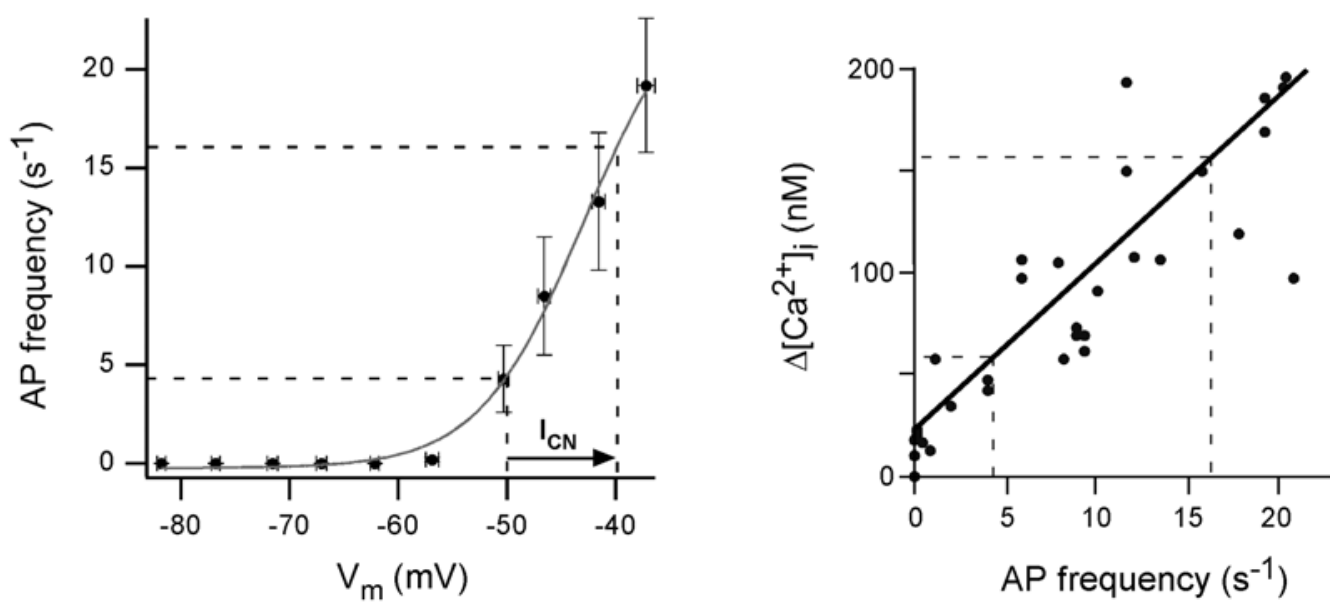

Fig. 3.3.2: $\boldsymbol{A}$ Monitoring of membrane potential $\left(\mathrm{V}_{\mathrm{m}}\right)$ and $\left[\mathrm{Ca}^{2+}\right]_{i}$ without TTX. The $\mathrm{CN}$-evoked depolarisation enhances action potential (AP) discharge, which elevates $\left[\mathrm{Ca}^{2+}\right]_{i}$ independently of the slow elevation described in the previous figure (arrow; APs were truncated). $\boldsymbol{B}$ The potential impact of $\mathrm{CN}$-induced depolarisations on $\left[\mathrm{Ca}^{2+}\right]_{i}$ was investigated in experiments, where AP firing was elicited by increasing current injection over a defined time interval $(5 \mathrm{~s})$ and corresponding $\left[\mathrm{Ca}^{2+}\right]_{i}$ changes were assessed $(n=9)$. The AP frequency $/ V_{m}$ relation (left) could be approximated by a sigmoid; $\left[\mathrm{Ca}^{2+}\right]_{i}$ changes in response to AP firing were well described by a linear function (right, correlation coefficient 0.86). The plots indicate that i) the $M N$ resting membrane potential is a critical determinant for $I_{\mathrm{CN}^{-}}$ induced increases in AP firing rates and subsequent $\mathrm{Ca}^{2+}$ influx, and ii) $I_{\mathrm{CN}}$-induced depolarisations can account for basal $\mathrm{Ca}^{2+}$ elevations of $\sim 100 \mathrm{nM}$, provided that resting membrane potential of cells is in the appropriate voltage range. (Intracelluar solutions contained $100 \mu \mathrm{M}$ fura-2)

A second source of $\mathrm{CN}$-dependent $\mathrm{Ca}^{2+}$ elevation was apparent when $\mathrm{I}_{\mathrm{CN}}$-mediated depolarisations evoked a series of action potentials (APs) as exemplified in Fig. 3.2.2 A. In this case, activation of voltage-dependent $\mathrm{Ca}^{2+}$ channels elevated cytosolic $\mathrm{Ca}^{2+}$ levels as previously investigated in great detail (Lips \& Keller, 1999). To evaluate the impact of $I_{C N}$ in a more systematic way, a series of depolarisations in 
current clamp mode (5 s) was performed and AP firing rates and corresponding changes in somatic $\left[\mathrm{Ca}^{2+}\right]$ were recorded $(n=9)$. As indicated in Fig. 3.3.2 B (left), AP firing was strongly dependent on $V_{m}$ and characteristic depolarisations of $10.2 \pm 1.1$ $\mathrm{mV}$ mediated by $\mathrm{I}_{\mathrm{CN}}$ corresponded to increases in AP firing rates from $4 \mathrm{~Hz}$ to $16 \mathrm{~Hz}$ in the voltage interval $-50 \mathrm{mV}$ to $-40 \mathrm{mV}$ (dashed lines). Correspondingly, these firing rates were associated with $\left[\mathrm{Ca}^{2+}\right]_{\mathrm{i}}$ elevations of $\sim 100 \mathrm{nM}$ as illustrated in Fig. 3.3.2 B (right). In summary, these observations illustrate that $\mathrm{i}$ ) the $\mathrm{MN}$ resting membrane potential is a critical determinant for $\mathrm{I}_{\mathrm{CN}}$-induced increases in AP firing rates and subsequent $\mathrm{Ca}^{2+}$ influx, and ii) $\mathrm{I}_{\mathrm{CN}}$-induced depolarisations can account for basal $\mathrm{Ca}^{2+}$ elevations of $100 \mathrm{nM}$, provided that resting membrane potential of cells is in the appropriate voltage range.

\subsection{2 $\mathrm{CN}$ releases $\mathrm{Ca}^{2+}$ from mitochondria-controlled store}

In subsequent experiments, we investigated the action potential-independent increase in $\left[\mathrm{Ca}^{2+}\right]$ in more detail. When HMs were held in voltage clamp (-60 or -70 $\mathrm{mV}$ ) in the presence of $0.5 \mu \mathrm{M}$ TTX, CN (2 mM for 45-70 s) produced a delayed rise in $\left[\mathrm{Ca}^{2+}\right]_{i}$ of $36 \pm 8 \mathrm{nM}(\mathrm{n}=10)$ that was comparable to the $\left[\mathrm{Ca}^{2+}\right]_{i}$ elevation observed under current clamp. Removal of $\mathrm{Ca}^{2+}$ from the extracellular solution did not prevent the increase in $\left[\mathrm{Ca}^{2+}\right]\left(n=6\right.$, Fig. 3.3.3 A), indicating that $\mathrm{Ca}^{2+}$ was released from intracellular stores.

$A$

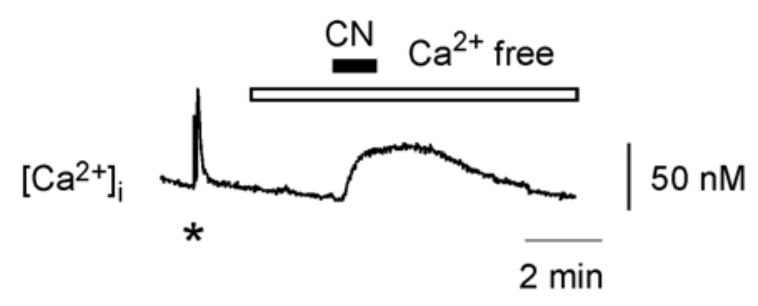

$B$

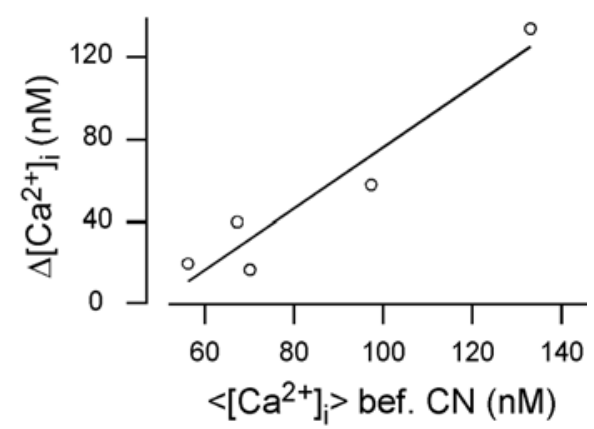

Fig. 3.3.3: $\boldsymbol{A}$ Monitoring of $\left[\mathrm{Ca}^{2+}\right]_{i}$ using fura-2 $(100 \mu \mathrm{M})$ in voltage-clamped hypoglossal MNs (HP -60 or $-70 \mathrm{mV})$. The asterisks marks a somatic $\mathrm{Ca}^{2+}$ transient produced by depolarisation to $+10 \mathrm{mV}(500$ $\mathrm{ms})$. During incubation with nominally $\mathrm{Ca}^{2+}$ free CSF (open bar), CN application (2 mM) increases resting $\left[\mathrm{Ca}^{2+}\right]_{\text {, }}$, indicating that $\mathrm{Ca}^{2+}$ is released from intracellular stores. $B$ The magnitude of $\mathrm{CN}$ induced $\mathrm{Ca}^{2+}$ release reflects the previous history of intracellular $\mathrm{Ca}^{2+}$ activity, which was determined by the average $\left[\mathrm{Ca}^{2+}\right]_{\mathrm{i}}\left(<\left[\mathrm{Ca}^{2+}\right]_{i}>\right)$ within 5 minutes before $\mathrm{CN}$ addition $(n=5$, correlation coefficient 0.96 ; $\mathrm{p}<0.05$, see Methods). Variations in $\left.<\left[\mathrm{Ca}^{2+}\right]\right\rangle$ were given by differential resting $\mathrm{Ca}^{2+}$ levels in the whole-cell configuration and a variable number ( 0 to 3 ) of depolarisations to $0 \mathrm{mV}(500 \mathrm{~ms})$ within the indicated time interval. 
As shown in Fig. 3.3.3 $\mathrm{B}$, the amount of releasable $\mathrm{Ca}^{2+}$ depended on the average $\left[\mathrm{Ca}^{2+}\right]_{i}$ before $\mathrm{CN}$ addition with a statistically significant correlation coefficient $\mathrm{r}_{0}=$ 0.96 (probability $\mathrm{p}\left(|\mathrm{r}| \geq \mathrm{r}_{0}\right)<0.05$, see also Methods). The average $\left[\mathrm{Ca}^{2+}\right]_{i}$ before $\mathrm{CN}$ addition was assessed within a time interval of 5 minutes from 5 different cells, where variations in $\left\langle\left[\mathrm{Ca}^{2+}\right]_{i}>\right.$ were given by differential resting $\mathrm{Ca}^{2+}$ levels in the whole-cell configuration and a variable number ( 0 to 3 ) of depolarisations to $0 \mathrm{mV}$ (500 ms) within the indicated time interval. Taken together, these experiments suggested that the $\mathrm{CN}$-sensitive store takes up $\mathrm{Ca}^{2+}$ during $\left[\mathrm{Ca}^{2+}\right]_{i}$ elevations and releases it during subsequent $\mathrm{CN}$ action.

Then, $I_{m}$ and $\left[\mathrm{Ca}^{2+}\right]_{i}$ of the patch-clamped cell were monitored together with NADH autofluorescence of neighbouring MNs. A series of experiments indicated that changes in $\mathrm{NADH}$ autofluorescence during $\mathrm{CN}$ exposure are extremely synchronous among $\mathrm{MNs}$ in the same area so that neighbouring $\mathrm{MNs}$ can be considered representative for the patch-clamped cell regarding this particular parameter. Recording of $\left[\mathrm{Ca}^{2+}\right]_{i}$ and NADH fluorescence in the same cell was not possible due to the overlapping spectra of fura- 2 and NADH. This experiment revealed that the $\mathrm{CN}$ induced release of $\mathrm{Ca}^{2+}$ followed the dynamics of mitochondrial metabolism very closely (Fig 3.3.4 A); the rise in $\left[\mathrm{Ca}^{2+}\right]_{i}$ followed the rise in NADH fluorescence with a constant temporal interval of $4 \pm 1 \mathrm{~s}(\mathrm{n}=12)$. As the $\mathrm{CN}$-induced accumulation of NADH is paralleled by depolarisation of $\Delta \psi$ (Fig. 3.2.6 A), the experiment suggested that $\mathrm{CN}$ released $\mathrm{Ca}^{2+}$ dependent on mitochondrial metabolism and $\Delta \psi$. This assumption was further confirmed by experiments where pre-incubation with the mitochondrial uncoupler carbonyl cyanide 4-trifluoro-methoxyphenylhydrazone (FCCP, $2 \mu \mathrm{M}$ ), which destroys $\Delta \psi$ and depletes the mitochondrial $\mathrm{Ca}^{2+}$ content, prevented the $\left[\mathrm{Ca}^{2+}\right]_{i}$ elevation during subsequent $\mathrm{CN}$ action $(n=2$, Fig. 3.3.4 B). 
$A$

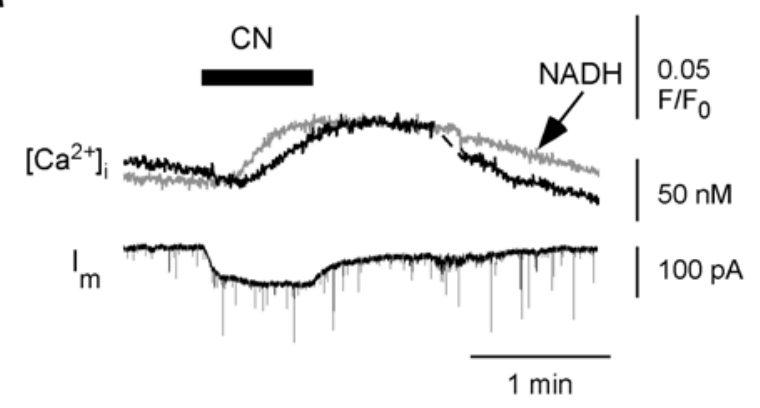

$C$

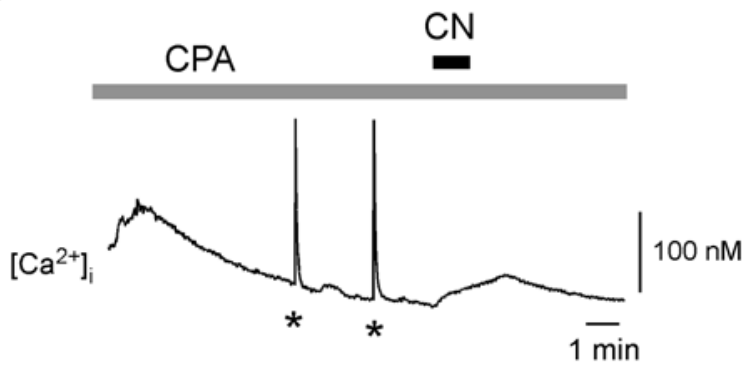

$B$

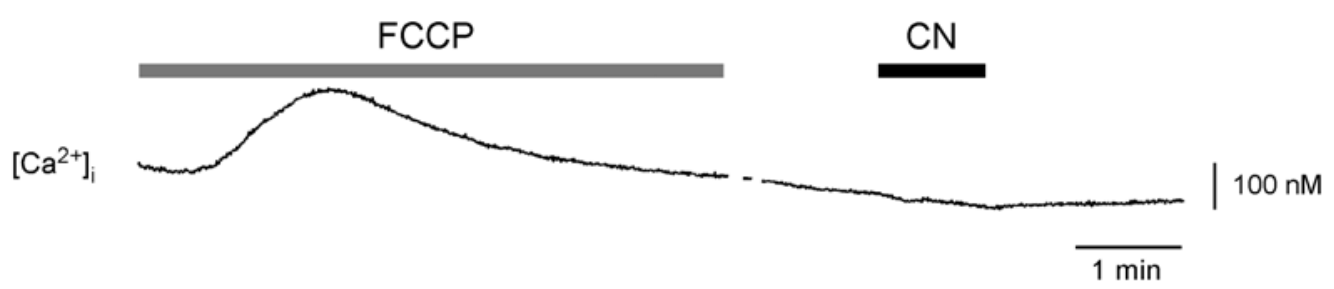

Fig. 3.3.4 A Simultaneous monitoring of membrane current $\left(\mathrm{I}_{\mathrm{m}}\right),\left[\mathrm{Ca}^{2+}\right]_{\mathrm{i}}$ and mitochondrial metabolism (NADH autofluorescence). Application of $\mathrm{CN}(2 \mathrm{mM})$ raises $\left[\mathrm{Ca}^{2+}\right]_{i}$ with kinetics that closely follow the changes in mitochondrial metabolism, suggesting that the $\mathrm{Ca}^{2+}$ was released from mitochondriacontrolled stores. B FCCP $(2 \mu \mathrm{M})$ was applied to destroy $\Delta \psi$ and to deplete mitochondrial $\mathrm{Ca}^{2+}$ content. Subsequent addition of $\mathrm{CN}$ does not change $\left[\mathrm{Ca}^{2+}\right]_{\mathrm{i}}$, confirming that the $\mathrm{CN}$-induced $\mathrm{Ca}^{2+}$ release was dependent on $\Delta \psi$. C Slices were incubated with CPA to inhibit the ER $\mathrm{Ca}^{2+}$-ATPase and to deplete $\mathrm{ER} \mathrm{Ca}{ }^{2+}$ content (CPA incubation started $\sim 25 \mathrm{~s}$ before onset of imaging). Voltage-induced $\mathrm{Ca}^{2+}$ transients were applied to fill mitochondrial stores (asterisks). Subsequent $\mathrm{CN}$ application raises $\left[\mathrm{Ca}^{2+}\right]_{\mathrm{i}}$, similar to the situation without CPA, indicating that the $\mathrm{CN}$-sensitive $\mathrm{Ca}^{2+}$ store was most likely represented by the mitochondria and not the ER.

However, in previous work it has been shown that both mitochondria and endoplasmic reticulum (ER) act as important $\mathrm{Ca}^{2+}$ buffers in hypoglossal MNs and that release from both stores is closely linked to $\Delta \psi$ (Ladewig et al., 2003). The observed $\mathrm{CN}$-induced $\mathrm{Ca}^{2+}$ release could therefore originate from both stores. To test a contribution of the ER, the ER $\mathrm{Ca}^{2+}$-ATPase was inhibited with $50 \mu \mathrm{M}$ cyclopiazonic acid (CPA) for at least 5 minutes, which released $\mathrm{Ca}^{2+}$ from the ER due to leakage of the ER membrane (Fig. 3.3.4 C). Control experiments showed that caffeine, which usually produces large $\mathrm{Ca}^{2+}$ elevations in hypoglossal MNs (Ladewig et al., 2003), did not invoke a rise in $\mathrm{Ca}^{2+}$, when CPA incubation preceded its action, indicating that CPA largely depleted the $\mathrm{ER} \mathrm{Ca}^{2+}$ content (not shown). When $\mathrm{CN}$ was then added after CPA pre-incubation, it still produced an increase in $\left[\mathrm{Ca}^{2+}\right]_{i}$ of $26 \pm 5$ $\mathrm{nM}(\mathrm{n}=6)$. Since the amount of $\mathrm{Ca}^{2+}$ release strongly depended on preceding cytosolic $\mathrm{Ca}^{2+}$ activity, it was difficult to compare this value with the control condition. However, these observations strongly suggest that the $\mathrm{CN}$-sensitive store, from which $\mathrm{Ca}^{2+}$ may be eventually released, is represented by the mitochondria. 


\subsubsection{Impact of $\mathrm{CN}$ on activity-dependent $\mathrm{Ca}^{2+}$ elevations}

As has been shown previously, hypoglossal MNs display repetitive elevations in $\left[\mathrm{Ca}^{2+}\right]_{\mathrm{i}}$, which are linked to rhythmic respiratory activity and mainly result from the opening of voltage-activated $\mathrm{Ca}^{2+}$ channels (Lips \& Keller, 1999; Ladewig \& Keller, 2000). A fast clearance of these repetitive elevations in $\left[\mathrm{Ca}^{2+}\right]_{i}$ is essential to maintain a low resting $\left[\mathrm{Ca}^{2+}\right]_{\mathrm{i}}$, particularly considering the low $\mathrm{Ca}^{2+}$ buffering capacity of hypoglossal MNs. As it is described in the first section of this work, mitochondrial $\mathrm{Ca}^{2+}$ uptake importantly contributes to a rapid clearance of cytosolic $\mathrm{Ca}^{2+}$ transients. Because mitochondrial $\mathrm{Ca}^{2+}$ uptake is dependent on the potential gradient over the inner mitochondrial membrane, depolarisation of $\Delta \psi$ as observed during $\mathrm{CN}$ action (Fig. 3.2.6) would predict disturbance in cytosolic $\mathrm{Ca}^{2+}$ clearance. To mimic the physiological situation, $\mathrm{Ca}^{2+}$ transients were repetitively elicited by short (200-500 $\mathrm{ms}$ ) depolarisations to $+10 \mathrm{mV}$ from a holding potential of $-60 \mathrm{mV}$ elevating $\left[\mathrm{Ca}^{2+}\right]_{i}$ to 200-500 nM from a resting level of $\sim 80 \mathrm{nM}$. Clearance of $\mathrm{Ca}^{2+}$ transients was assessed by determining the recovery time constant tau $(\tau)$ after fitting with a singleexponential function. As expected, incubation with 1-2 $\mathrm{mM} \mathrm{CN}$ for 1 to 4 minutes markedly prolonged the recovery of somatic $\mathrm{Ca}^{2+}$ transients to $1.96 \pm 0.35$ fold control ( $n=6, p<0.03$; Fig. 3.3.5 A). After wash-out of $\mathrm{CN}$, fast clearance of $\mathrm{Ca}^{2+}$ transients was largely restored.

In these experiments, ATP-deficiency as a causative factor for prolongation of recovery times was ruled out, since millimolar ATP concentrations were continuously supplied via the patch pipette. However, a decrease in cellular ATP levels is certainly expected during persistent inhibition of mitochondrial electron transport during in vivo conditions of hypoxia and may affect $\left[\mathrm{Ca}^{2+}\right]_{i}$ in addition. To test the impact of ATPdepletion on regulation of $\left[\mathrm{Ca}^{2+}\right]_{i}$ and recovery of activity-dependent $\mathrm{Ca}^{2+}$ transients, $\left[\mathrm{Ca}^{2+}\right]_{\mathrm{i}}$ was monitored in cells dialysed with ATP-free intracellular solution and $\mathrm{Ca}^{2+}$ transients were elicited every $60 \mathrm{~s}$. Oligomycin $(10 \mu \mathrm{g} / \mathrm{ml})$ was then added to block mitochondrial ATP-production. In contrast to $\mathrm{CN}$, oligomycin does not depolarise $\Delta \psi$, which was consistent with a lacking effect on the NADH autofluorescence (not shown). As illustrated in Fig. 3.3.5 B, wash-in of oligomycin left basal $\mathrm{Ca}^{2+}$ levels, as well as recovery times of $\mathrm{Ca}^{2+}$ transients, unaffected for several minutes, before recovery times progressively prolonged and, after a mean incubation time of $6.4 \pm 1.2$ min, resting $\left[\mathrm{Ca}^{2+}\right]_{i}$ steadily increased $(n=6)$. 
A
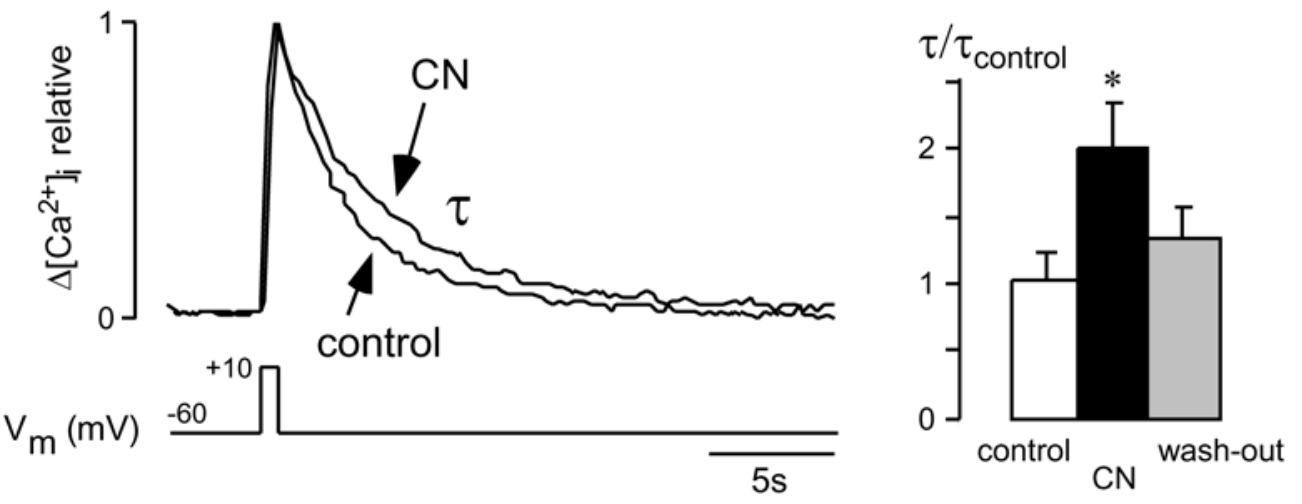

B
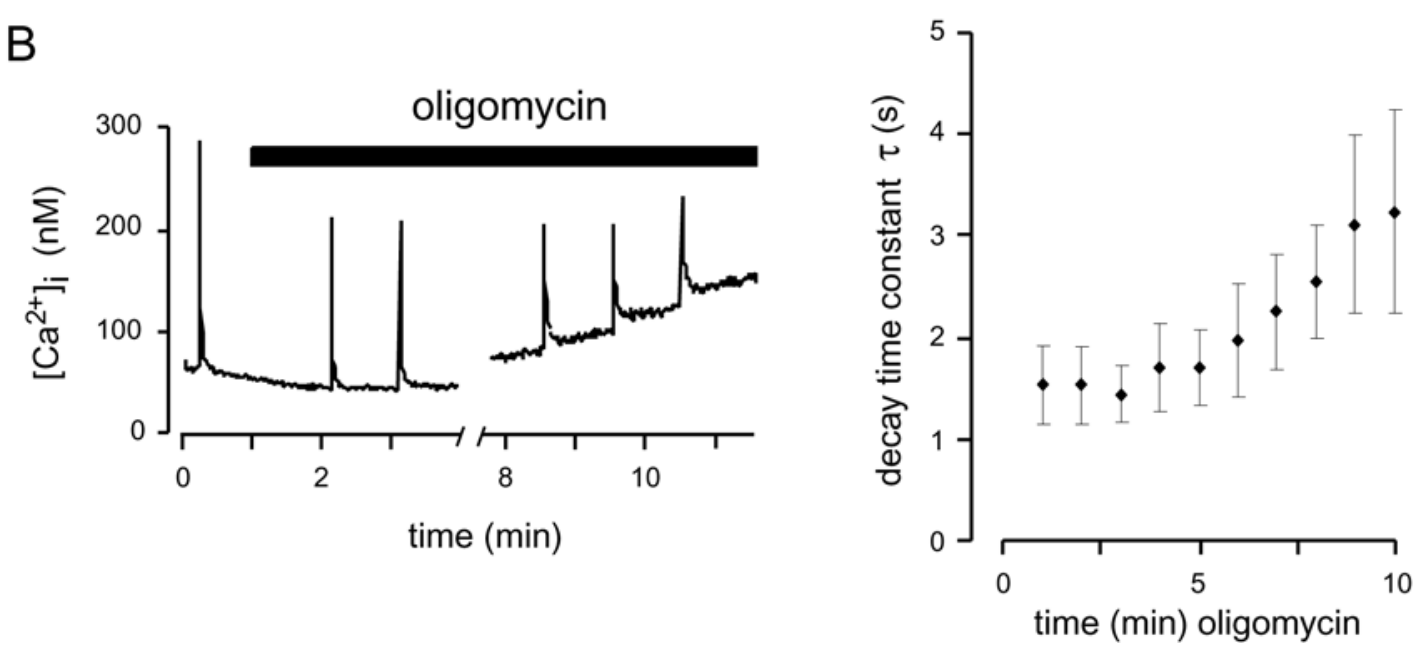

Fig. 3.3.5: Monitoring of $\left[\mathrm{Ca}^{2+}\right]_{i}$ in patch-clamped hypoglossal MNs using fura-2 (100 $\left.\mu \mathrm{M}\right)$, where elevations in $\left[\mathrm{Ca}^{2+}\right]_{\mathrm{i}}(100-400 \mathrm{nM}$ above baseline) were elicited by short depolarisations to $+10 \mathrm{mV}$ from a holding potential of -60 or $-70 \mathrm{mV}$. A Somatic $\mathrm{Ca}^{2+}$-transients normalized to the same amplitude before and during 1-4 minutes of $\mathrm{CN}$ incubation $(1 \mathrm{mM})$ are superimposed. Note that $\mathrm{CN}$ prolongs the recovery time constant tau $(\tau)$ of $\mathrm{Ca}^{2+}$-transients to $1.96 \pm 0.35$ fold control $(n=6 ; p<0.03)$. Wash-out of $\mathrm{CN}$ largely restores fast $\mathrm{Ca}^{2+}$ clearance. Because cells were dialysed with $4 \mathrm{mM} \mathrm{ATP}$, the effect is attributable to disturbed mitochondrial $\mathrm{Ca}^{2+}$ uptake and not to ATP-depletion. $\boldsymbol{B}$ Hypoglossal MNs were dialysed with ATP-free intracellular solution and $10 \mu \mathrm{g} / \mathrm{ml}$ oligomycin was added as indicated to block mitochondrial ATP production. While usually no change in recovery time constants of $\mathrm{Ca}^{2+}$ transients $(\tau)$ or resting $\left[\mathrm{Ca}^{2+}\right]_{i}$ was noticed within the first 5 minutes, longer incubation times progressively prolonged $\tau$. After a mean incubation time of $6.4 \pm 1.2 \mathrm{~min}$, resting $\left[\mathrm{Ca}^{2+}\right]_{\mathrm{i}}$ steadily increased. 


\section{DISCUSSION}

\subsection{Dominant role of mitochondria in clearance of physiological $\mathrm{Ca}^{2+}$ loads in}

\section{hypoglossal motoneurons}

The first part of this work has characterized the contribution of mitochondria to buffering of voltage-activated $\mathrm{Ca}^{2+}$ loads in hypoglossal motoneurons. The experiments indicated that mitochondria contribute importantly to a fast clearance of $\mathrm{Ca}^{2+}$ transients by taking up around $50 \%$ of motoneuron $\mathrm{Ca}^{2+}$ loads even for $\left[\mathrm{Ca}^{2+}\right]_{\mathrm{i}}$ elevations with amplitudes as small as 50-200 nM (physiological range). By taking up $\mathrm{Ca}^{2+}$, mitochondria also contribute to limiting the amplitude of voltage-activated $\mathrm{Ca}^{2+}$ elevations, which was revealed in experiments using dyes with lower affinity. These results are in good agreement with data obtained from motor nerve terminals (David \& Barrett, 2000), with experiments on cultured motoneurons (Carriedo et al., 2000) and with a previous study on patch-clamped hypoglossal motoneurons (Ladewig et al., 2003), where significant mitochondrial $\mathrm{Ca}^{2+}$ uptake following voltage-activated $\mathrm{Ca}^{2+}$ influx has also been demonstrated. The fact that the study by Ladewig et al. yielded very similar results indicates that whole-cell patch-clamping is unlikely to disturb mitochondrial $\mathrm{Ca}^{2+}$ buffering in motoneurons.

Further experiments demonstrated that mitochondrial $\mathrm{Ca}^{2+}$ uptake is highly temperature-dependent in motoneuron somata with $\sim 2$ fold higher uptake rate at 29$32^{\circ} \mathrm{C}$ compared to room temperature. This finding is consistent with a study from David \& Barret (2000) showing that mitochondrial $\mathrm{Ca}^{2+}$ buffering on mouse motor nerve terminals is more efficient at near physiological temperatures than at room temperature. However, in contrast to their results, the relative contribution of mitochondria to clearance of cytosolic $\mathrm{Ca}^{2+}$ loads remained essentially constant in the tested temperature range. This indicated that experiments performed at room temperature still yield representative results regarding mitochondrial $\mathrm{Ca}^{2+}$ clearance in this preparation.

Quantitative information about total motoneuron $\mathrm{Ca}^{2+}$ clearance and mitochondrial $\mathrm{Ca}^{2+}$ uptake was obtained by calculation of clearance rates after polynomial fitting of the decay of $\mathrm{Ca}^{2+}$ transients (amplitudes up to $350 \mathrm{nM}$ ). The resulting clearance rates 
for the undisturbed condition (average $\gamma_{\text {total }} 152 \mathrm{~s}^{-1}$ ) were found to be slightly higher than those calculated previously (Lips \& Keller, 1999; see also Methods section 2.9), a fact that may result from differential composition of intracellular solutions or variable preservation of MNs in the slice. Mitochondrial $\mathrm{Ca}^{2+}$ clearance was characterized by a clearance rate constant of $\sim 70 \mathrm{~s}^{-1}$. This value (room temperature) and the characteristic $\sim 2$ fold increase in time integral of $\left[\mathrm{Ca}^{2+}\right]_{i}$ transients after FCCP could be used to compare mitochondrial $\mathrm{Ca}^{2+}$ buffering between different cellular systems. This revealed that mitochondrial $\mathrm{Ca}^{2+}$ buffering of small amplitude elevations is much more prominent in motoneurons than other neuronal systems, such as sympathetic neurons (Friel \& Tsien, 1994), dorsal root ganglion neurons (Werth \& Thayer, 1994), hippocampal neurons (Mironov, 1995) or chromaffin cells (Herrington et al., 1996; Xu et al., 1997).

\subsection{Mechanisms underlying efficient mitochondrial $\mathrm{Ca}^{2+}$ uptake}

An interesting question was therefore, how such efficient mitochondrial $\mathrm{Ca}^{2+}$ uptake at low $\left[\mathrm{Ca}^{2+}\right]_{i}$ can be reached in hypoglossal motoneurons and several explanations were considered. One possibility was that motoneuron mitochondria - in contrast to other neurons - take up $\mathrm{Ca}^{2+}$ preferentially via the "rapid uptake mode" (RaM). This mode has been described to account for efficient $\mathrm{Ca}^{2+}$ uptake during trains of $\mathrm{Ca}^{2+}$ pulses of physiological concentration $(\sim 400 \mathrm{nM})$ in isolated mitochondria (Gunter et al., 1998). It was suggested that RaM is mediated by a specific transport mechanism that might be responsible for mitochondrial $\mathrm{Ca}^{2+}$ uptake from $\left[\mathrm{Ca}^{2+}\right]_{\mathrm{i}}$ transients in vivo. However, there is little evidence for existence of RaM in intact neurons and no statement about contribution of RaM in motoneurons can be made.

In contrast, mechanisms potentially contributing to mitochondrial $\mathrm{Ca}^{2+}$ uptake at low $\left[\mathrm{Ca}^{2+}\right]_{\mathrm{i}}$ that are supported by experimental evidence include i) a high density of mitochondria in the cytosol correlating to a high level of mitochondrial activity, and ii) a significant interaction of mitochondria and areas of $\mathrm{Ca}^{2+}$ accumulation around $\mathrm{Ca}^{2+}$ influx pathways. Previous studies already indicated a high level of mitochondrial activity in motoneurons (Wong-Riley, 1989; Shaw \& Eggett, 2000) and this was now confirmed by rhod123 staining, cytochrome oxidase histochemistry and two-photon $\mathrm{NADH}$ imaging for the hypoglossal nucleus. The possibility (ii) that mitochondrial $\mathrm{Ca}^{2+}$ uptake was facilitated by a close proximity of mitochondria and $\mathrm{Ca}^{2+}$ influx sites was 
supported by confocal laser scanning using mitotracker green, although the spatial resolution reached in these experiments restricted statements to the $\mu \mathrm{m}$ range. However, additional support is provided by experiments, in which the low affinity dye fura-FF was employed. This demonstrated an increase in $\mathrm{Ca}^{2+}$ transient amplitudes after mitochondrial depolarisation, which was not seen when fura-2 was used. The most likely explanation for the observed phenomenon was that fura-2 saturated at high $\left[\mathrm{Ca}^{2+}\right]$ and therefore failed to detect an increase in transient amplitudes after FCCP. As the bulk cytosolic $\left[\mathrm{Ca}^{2+}\right]$ did not exceed $500 \mathrm{nM}$ during stimulation, saturation of fura-2 occurred most likely locally, in areas of $\mathrm{Ca}^{2+}$ accumulation around $\mathrm{Ca}^{2+}$ influx sites (voltage-dependent $\mathrm{Ca}^{2+}$ channels). This was even more likely as the low cytosolic $\mathrm{Ca}^{2+}$ buffering capacity of hypoglossal motoneurons significantly enhances formation of spatial gradients. Therefore, a model seems reasonable where a fraction of motoneuron mitochondria interacts with areas of high $\left[\mathrm{Ca}^{2+}\right]$ around influx sites (Fig. 4.1).

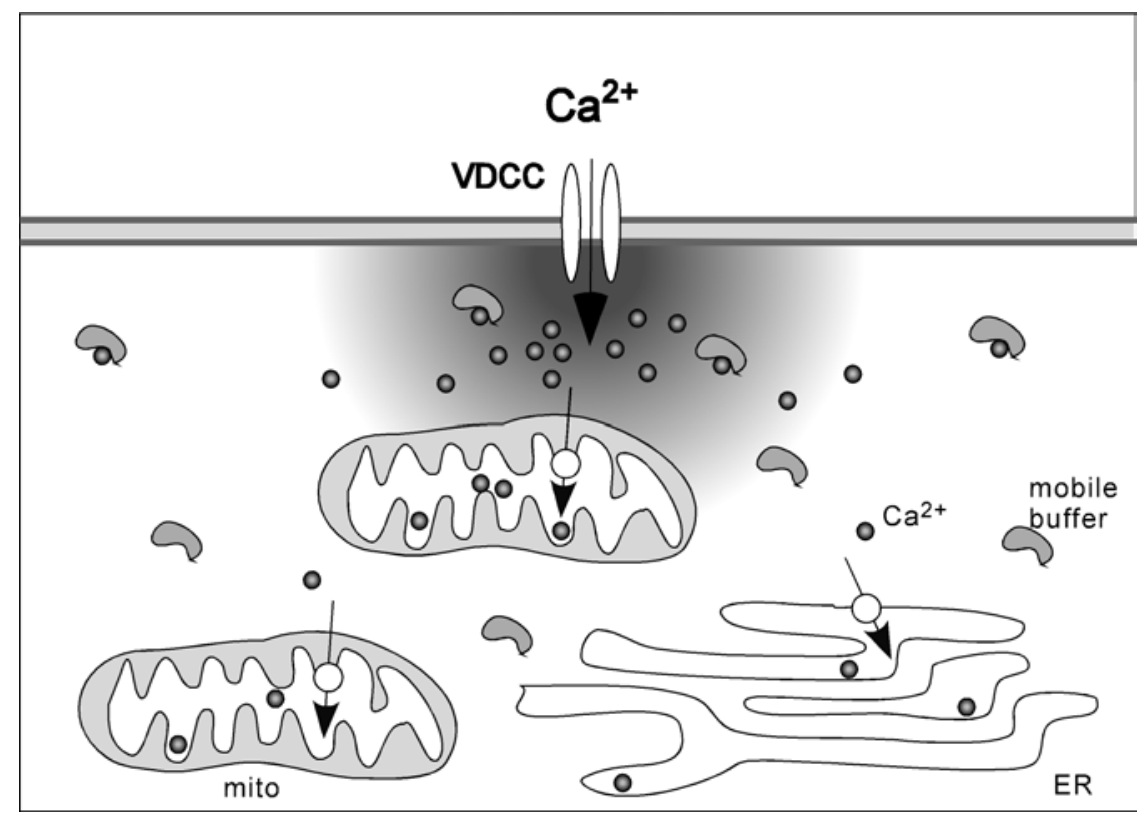

Fig. 4.1: Model of interaction between mitochondria and areas of high $\left[\mathrm{Ca}^{2+}\right]$ around voltagedependent $\mathrm{Ca}^{2+}$ channels (VDCC).

The proposed model seems even more convincing considering that the picture of mitochondria as "biosensors of calcium microdomains" has emerged from a variety of studies (Lawrie et al., 1996; Rizzuto et al., 1999). This model also nicely fits with previous observations on hypoglossal motoneurons, where highly localized regulation of store-dependent $\mathrm{Ca}^{2+}$ profiles was revealed by two-photon microscopy (Ladewig et al., 2003). The model is also consistent with the finding that a rise in mitochondrial $\left[\mathrm{Ca}^{2+}\right]$ following voltage-activated $\mathrm{Ca}^{2+}$ influx is not delayed to the 
increase in cytosolic $\left[\mathrm{Ca}^{2+}\right.$ ] (Ladewig et al., 2003). Although more studies are necessary to confirm interaction of mitochondria and local $\mathrm{Ca}^{2+}$ domains, the results support the assumption that $\mathrm{Ca}^{2+}$ accumulation around influx sites facilitates mitochondrial $\mathrm{Ca}^{2+}$ uptake in hypoglossal motoneurons and this, in addition to other mechanisms, may contribute to efficient mitochondrial $\mathrm{Ca}^{2+}$ uptake even when the bulk cytosolic $\left[\mathrm{Ca}^{2+}\right]$ does not exceed $500 \mathrm{nM}$.

\subsection{Consequences of disturbed mitochondrial metabolism}

The aim of the second part of the study was to define the impact of mitochondrial dysfunction - as observed in forms of ALS - on motoneuron physiology. For this purpose, complex IV of the respiratory chain was inhibited with cyanide (CN) and the consequences were investigated with particular attention to membrane currents and $\left[\mathrm{Ca}^{2+}\right]_{\text {. }}$. A summary of the mechanisms identified is given in Fig. 4.2.

The effect of $\mathrm{CN}$ on motoneuron membrane properties was characterized by opening of TTX-insensitive $\mathrm{Na}^{+}$conductances $\left(\mathrm{I}_{\mathrm{CN}}\right)$, which depolarised the resting membrane potential $\left(\mathrm{V}_{\mathrm{m}}\right)$ of motoneurons by $\sim 10 \mathrm{mV}$ and increased action potential activity. This response very much resembled the depolarisation of motoneurons during hypoxia (Haddad \& Jiang, 1993), a fact that is not surprising considering that oxygen deprivation also inhibits the respiratory chain on the level of complex IV. Further experiments indicated that a redox mechanism was involved in activation of $\mathrm{I}_{\mathrm{CN}}$, because the antioxidants and free radical scavengers ascorbic acid and trolox ${ }^{\circledR}$ largely prevented it. The change in the redox state was possibly induced by increased levels of reactive oxygen species (ROS), which originate at the respiratory chain when complex IV activity is inhibited (Lopez-Barneo et al., 2001). However, the involvement of ROS is challenged by the fact that the activation of $I_{C N}$ significantly preceded the increase in NADH fluorescence, which indirectly monitored the build up of electrons at the respiratory chain. Although a very localized production of ROS that was not represented by the global NADH signal could account for the observed discrepancy, alternate models for $I_{\mathrm{CN}}$ activation should be considered. For example, a molecule other than complex IV could serve as sensor capable of responding to hypoxia, $\mathrm{CN}$ and azide, another complex IV inhibitor that mimicked the $\mathrm{CN}$ response. In this case, the redox state of a thiol-rich molecule as part of the channel itself or of a regulatory protein could constitute the sensor similar as it was suggested by 
Hammerström and Gage (1998; 2000) for oxygen and CN sensing $\mathrm{Na}^{+}$channels in hippocampal neurons. Taken together, despite the plausible involvement of ROS in activation of $\mathrm{I}_{\mathrm{CN}}$, more experiments are necessary to determine the exact signalling pathway by which $\mathrm{I}_{\mathrm{CN}}$ is mediated in MNs.

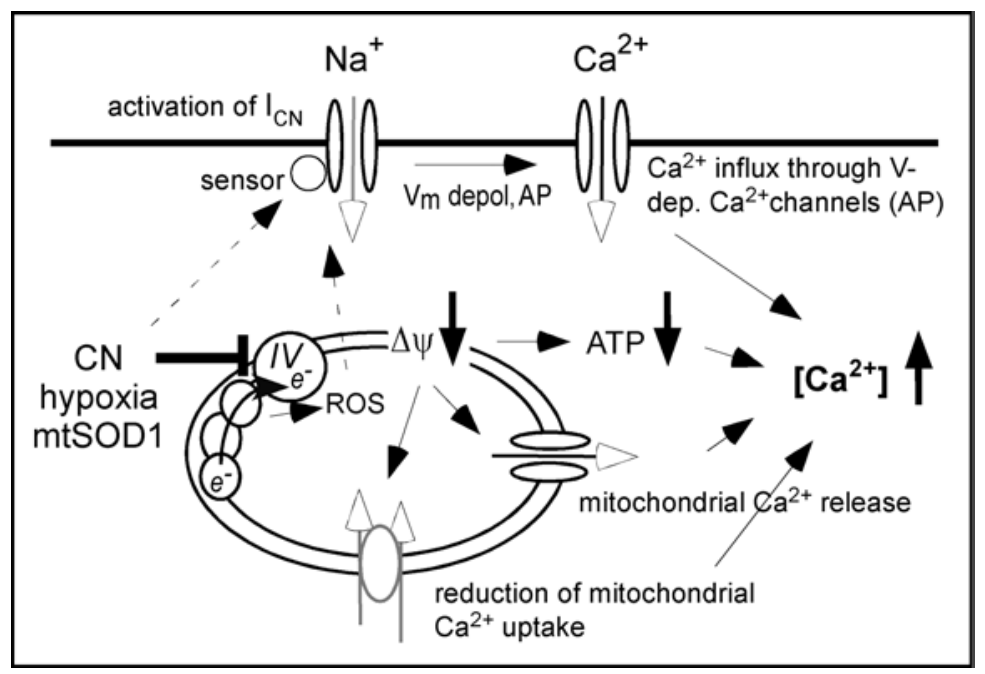

Fig. 4.2: Events following mitochondrial inhibition in vulnerable hypoglossal MNs: $\mathrm{CN}$ (similar to hypoxia and mtSOD1) inhibits complex IV (cytochrome $c$ oxidase) of the respiratory chain. This increases the formation of reactive oxygen species (ROS), which potentially activate a depolarising $\mathrm{Na}^{+}$current $\left(I_{C N}\right)$. Alternatively, the $\mathrm{Na}^{+}$current is activated by a direct redox mechanism. The $\mathrm{Na}^{+}$ influx enhances the neuronal excitability and promotes $\mathrm{Ca}^{2+}$ influx during elevated firing rates (action potentials, AP). Inhibition of the respiratory chain furthermore decreases the mitochondrial potential gradient $(\Delta \psi)$ leading to reduced $\mathrm{Ca}^{2+}$ uptake into the mitochondrial matrix and release of $\mathrm{Ca}^{2+}$ that was taken up during preceding activity. Mitochondrial inhibition additionally decreases cellular ATP levels, and this further enhances accumulation of intracellular $\mathrm{Ca}^{2+}$.

In addition to the changes in electrical properties, several mechanisms were identified, by which mitochondrial inhibition increased the cytosolic $\mathrm{Ca}^{2+}$ load of hypoglossal motoneurons. Attributable to dissipation of the proton gradient $(\Delta \psi)$, we observed release of $\mathrm{Ca}^{2+}$ from mitochondria-controlled stores and a notable retardation of cytosolic $\mathrm{Ca}^{2+}$ clearance rates. Additionally, voltage-dependent $\mathrm{Ca}^{2+}$ influx increased $\left[\mathrm{Ca}^{2+}\right]_{i}$ during elevated firing rates. Since millimolar ATP concentrations were continuously supplied via the patch pipette, these effects were clearly independent of a drop in cytosolic ATP levels. On the other hand, a decrease in cellular ATP concentration, which was induced by oligomycin application while respiratory chain activity continued in another set of experiments, prolonged the recovery times of $\mathrm{Ca}^{2+}$ transients and progressively built up basal $\left[\mathrm{Ca}^{2+}\right]_{i}$ with a delay of 5-6 minutes. These observations clearly indicated that early cytosolic $\mathrm{Ca}^{2+}$ disturbances during mitochondrial inhibition do not arise from energy depletion but rather from insufficient mitochondrial $\mathrm{Ca}^{2+}$ buffering and changes in the neuronal 
excitability. Nevertheless, when cytosolic ATP levels drop below a certain critical value, $\left[\mathrm{Ca}^{2+}\right]_{\mathrm{i}}$ regulation is even more severely impaired.

\subsection{Selective vulnerability of motoneurons}

The reason why motoneuron populations are preferentially injured in ALS is still incompletely understood, and a variety of explanations have been proposed. Motoneurons are large, highly active cells with exceptional energy requirements, a fact that exposes them to elevated risks during metabolic demands. They also possess a high level of $\mathrm{Ca}^{2+}$ permeable AMPA receptors and a remarkably low cytosolic $\mathrm{Ca}^{2+}$ buffering capacity $\left(\mathrm{K}_{\mathrm{S}}\right)$ (Alexianu et al., 1994; Lips \& Keller, 1998; Palecek et al., 1999), which renders them particularly sensitive to excitatory glutamatergic transmission. This work now establishes that motoneurons are further characterized by a strong impact of mitochondria on $\left[\mathrm{Ca}^{2+}\right]_{i}$ transients. This fact might enhance their vulnerability for several reasons. First, large $\mathrm{Ca}^{2+}$ fluxes into mitochondria enhance the risk for generation of reactive oxygen species, molecules that promote oxidative damage to the cell (Nicholls \& Budd, 2000). Additionally, the strong mitochondrial control of $\left[\mathrm{Ca}^{2+}\right]_{\mathrm{i}}$ increases the risk for disturbance of $\mathrm{Ca}^{2+}$ homeostasis, once mitochondrial function is impaired.

The study furthermore indicates that vulnerable MNs are characterized by membrane properties, which promote a profound depolarisation during mitochondrial inhibition. In contrast, neurons in the nucleus dorsalis vagus, which are tolerant to hypoxia and also resistant to degeneration in ALS, hyperpolarise under the same conditions. Hyperpolarisation in vagal but also in hippocampal neurons in response to mitochondrial inhibition has been attributed to activation of ATP-sensitive and $\mathrm{Ca}^{2+}$ dependent $\mathrm{K}^{+}$channels (Koyama et al., 1999; Englund et al., 2001; Müller et al., 2002). Although activation of ATP-dependent $\mathrm{K}^{+}$channels during mitochondrial inhibition has also been observed in hypoglossal MNs (Jiang \& Haddad, 1991), its activation is apparently not sufficient to counteract the depolarisation induced by $\mathrm{Na}^{+}$ influx. Taken together, these observations therefore suggest that selective MN vulnerability most likely results from a synergistic accumulation of risk factors, including low $\mathrm{Ca}^{2+}$ buffering, strong mitochondrial control of $\left[\mathrm{Ca}^{2+}\right]_{i}$ and a weak protection against hypoxia-related changes in neuronal excitability. 


\subsection{Implications for the pathogenesis of ALS}

Inhibition of the respiratory chain and mitochondrial dysfunction have been closely linked to the pathogenesis of ALS. This study has defined some of the pathophysiological changes resulting from mitochondrial inhibition such as an increase in excitability and cytosolic $\mathrm{Ca}^{2+}$ load in vulnerable motoneurons. Although very young animals were used in this study and the cellular changes were investigated over a time range of minutes whereas ALS damages motoneurons over months, the findings may have implications for motoneuron degeneration in ALS. Several observations support this assumption. For example, increased cytosolic $\mathrm{Ca}^{2+}$ loads resulting from mitochondrial inhibition are paralleled by observations on cell lines expressing mutant SOD1, which also show increased basal $\mathrm{Ca}^{2+}$ loads (Carri et al., 1997; Kruman et al., 1999). Moreover, the findings of depolarising $\mathrm{Na}^{+}$currents during complex IV inhibition resemble increased $\mathrm{Na}^{+}$currents and enhanced neuronal excitability in mutant SOD1 mouse spinal motoneurons (Kuo et al., 2002; Kuo et al., 2003). Although a causal link cannot be drawn, is seems plausible that increased excitability and increased $\mathrm{Ca}^{2+}$ loads are critical determinants of motoneuron degeneration. For example, a mitochondrial-dependent increase in motoneuron excitability may well explain the observed sensitisation to excitatory glutamatergic transmission that has been implicated in mutant SOD1-based ALS (Roy et al., 1998). Substantial rises in cytosolic $\left[\mathrm{Ca}^{2+}\right]$ may trigger secondary $\mathrm{Ca}^{2+}$ dependent phenomena, which promote neurotoxicity and eventually result in cell death. This includes activation of the $\mathrm{Ca}^{2+}$ dependent protease calpain and endonucleases, which promote cytoskeletal breakdown and DNA degradation. Accordingly, the potential link between motoneuron responses to mitochondrial inhibition and their selective vulnerability during ALS-related motoneuron disease will be an interesting area for future investigation. 


\section{LITERATURE}

Alexianu ME, Ho BK, Mohamed AH, La Bella V, Smith RG \& Appel SH (1994). The role of calcium-binding proteins in selective motoneuron vulnerability in amyotrophic lateral sclerosis. Ann Neurol 36, 846-858.

Appel SH, Smith RG, Engelhardt JI \& Stefani E (1994). Evidence for autoimmunity in amyotrophic lateral sclerosis. J Neurol Sci 124 Suppl, 14-19.

Babcock DF, Herrington J, Goodwin PC, Park YB \& Hille B (1997). Mitochondrial participation in the intracellular $\mathrm{Ca}^{2+}$ network. $J$ Cell Biol 136, 833-844.

Beers DR, Ho BK, Siklos L, Alexianu ME, Mosier DR, Mohamed AH, Otsuka Y, Kozovska ME, McAlhany RE, Smith RG \& Appel SH (2001). Parvalbumin overexpression alters immune-mediated increases in intracellular calcium, and delays disease onset in a transgenic model of familial amyotrophic lateral sclerosis. J Neurochem 79, 499-509.

Borthwick GM, Johnson MA, Ince PG, Shaw PJ \& Turnbull DM (1999). Mitochondrial enzyme activity in amyotrophic lateral sclerosis: implications for the role of mitochondria in neuronal cell death. Ann Neurol 46, 787-790.

Bowling AC, Barkowski EE, McKenna-Yasek D, Sapp P, Horvitz HR, Beal MF \& Brown RH, Jr. (1995). Superoxide dismutase concentration and activity in familial amyotrophic lateral sclerosis. J Neurochem 64, 2366-2369.

Bruijn LI, Houseweart MK, Kato S, Anderson KL, Anderson SD, Ohama E, Reaume AG, Scott RW \& Cleveland DW (1998). Aggregation and motor neuron toxicity of an ALS-linked SOD1 mutant independent from wild-type SOD1. Science 281, 1851-1854.

Budd SL \& Nicholls DG (1996). A reevaluation of the role of mitochondria in neuronal $\mathrm{Ca}^{2+}$ homeostasis. J Neurochem 66, 403-411.

Carri MT, Ferri A, Battistoni A, Famhy L, Gabbianelli R, Poccia F \& Rotilio G (1997). Expression of a $\mathrm{Cu}, \mathrm{Zn}$ superoxide dismutase typical of familial amyotrophic lateral sclerosis induces mitochondrial alteration and increase of cytosolic $\mathrm{Ca}^{2+}$ concentration in transfected neuroblastoma SH-SY5Y cells. FEBS Lett 414, 365-368.

Carriedo SG, Sensi SL, Yin HZ \& Weiss JH (2000). AMPA exposures induce mitochondrial $\mathrm{Ca}(2+)$ overload and $\mathrm{ROS}$ generation in spinal motor neurons in vitro. J Neurosci 20, 240-250.

Carriedo SG, Yin HZ \& Weiss JH (1996). Motor neurons are selectively vulnerable to AMPA/kainate receptor-mediated injury in vitro. J Neurosci 16, 4069-4079.

David G \& Barrett EF (2000). Stimulation-evoked increases in cytosolic [Ca(2+)] in mouse motor nerve terminals are limited by mitochondrial uptake and are temperature-dependent. J Neurosci 20, 7290-7296. 
David G, Barrett JN \& Barrett EF (1998). Evidence that mitochondria buffer physiological $\mathrm{Ca}^{2+}$ loads in lizard motor nerve terminals. J Physiol 509, 59-65.

Duchen MR (2000). Mitochondria and calcium: from cell signalling to cell death. J Physiol 529 Pt 1, 57-68.

Engelhardt JI, Siklos L, Komuves L, Smith RG \& Appel SH (1995). Antibodies to calcium channels from ALS patients passively transferred to mice selectively increase intracellular calcium and induce ultrastructural changes in motoneurons. Synapse 20, 185-199.

Englund M, Hyllienmark L \& Brismar T (2001). Chemical hypoxia in hippocampal pyramidal cells affects membrane potential differentially depending on resting potential. Neuroscience 106, 89-94.

Fierro L, DiPolo R \& Llano I (1998). Intracellular calcium clearance in Purkinje cell somata from rat cerebellar slices. J Physiol 510 ( Pt 2), 499-512.

Friel DD \& Tsien RW (1994). An FCCP-sensitive Ca2+ store in bullfrog sympathetic neurons and its participation in stimulus-evoked changes in $\left[\mathrm{Ca}^{2+}\right]$ i. $\mathrm{J}$ Neurosci $14,4007-4024$.

Grynkiewicz G, Poenie M \& Tsien RY (1985). A new generation of $\mathrm{Ca}^{2+}$ indicators with greatly improved fluorescence properties. J Biol Chem 260, 3440-3450.

Gunter TE, Buntinas L, Sparagna GC \& Gunter KK (1998). The $\mathrm{Ca}^{2+}$ transport mechanisms of mitochondria and $\mathrm{Ca} 2+$ uptake from physiological-type $\mathrm{Ca}^{2+}$ transients. Biochim Biophys Acta 1366, 5-15.

Gurney ME, Pu H, Chiu AY, Dal Canto MC, Polchow CY, Alexander DD, Caliendo J, Hentati A, Kwon YW, Deng HX, Chen W, Zhai P, Sufit RL \& Siddique T (1994). Motor neuron degeneration in mice that express a human $\mathrm{Cu}, \mathrm{Zn}$ superoxide dismutase mutation. Science 264, 1772-1775.

Haddad GG \& Jiang C (1993). Mechanisms of anoxia-induced depolarization in brainstem neurons: in vitro current and voltage clamp studies in the adult rat. Brain Res 625, 261-268.

Hamill OP, Marty A, Neher E, Sakmann B \& Sigworth FJ (1981). Improved patchclamp techniques for high-resolution current recording from cells and cell-free membrane patches. Pflugers Arch 391, 85-100.

Hammarström AK \& Gage PW (1998). Inhibition of oxidative metabolism increases persistent sodium current in rat CA1 hippocampal neurons. J Physiol 510, 735-741.

Hammarström AK \& Gage PW (2000). Oxygen-sensing persistent sodium channels in rat hippocampus. J Physiol $529 \mathrm{Pt} 1,107-118$. 
Heath PR \& Shaw PJ (2002). Update on the glutamatergic neurotransmitter system and the role of excitotoxicity in amyotrophic lateral sclerosis. Muscle Nerve 26, 438-458.

Hernandez-Guijo JM, Maneu-Flores VE, Ruiz-Nuno A, Villarroya M, Garcia AG \& Gandia L (2001). Calcium-dependent inhibition of $L, N$, and P/Q Ca2+ channels in chromaffin cells: role of mitochondria. J Neurosci 21, 2553-2560.

Herrington J, Park YB, Babcock DF \& Hille B (1996). Dominant role of mitochondria in clearance of large $\mathrm{Ca}^{2+}$ loads from rat adrenal chromaffin cells. Neuron 16, 219-228.

Ho BK, Alexianu ME, Colom LV, Mohamed AH, Serrano F \& Appel SH (1996). Expression of calbindin-D28K in motoneuron hybrid cells after retroviral infection with calbindin-D28K cDNA prevents amyotrophic lateral sclerosis IgG-mediated cytotoxicity. Proc Natl Acad Sci U S A 93, 6796-6801.

Jiang C \& Haddad GG (1991). Effect of anoxia on intracellular and extracellular potassium activity in hypoglossal neurons in vitro. J Neurophysiol 66, 103-111.

Julien JP, Couillard-Despres S \& Meier J (1998). Transgenic mice in the study of ALS: the role of neurofilaments. Brain Pathol 8, 759-769.

Jung C, Higgins CM \& Xu Z (2002). Mitochondrial electron transport chain complex dysfunction in a transgenic mouse model for amyotrophic lateral sclerosis. $J$ Neurochem 83, 535-545.

Kaal EC, Vlug AS, Versleijen MW, Kuilman M, Joosten EA \& Bar PR (2000). Chronic mitochondrial inhibition induces selective motoneuron death in vitro: a new model for amyotrophic lateral sclerosis. J Neurochem 74, 1158-1165.

Kawai Y, Qi J, Comer AM, Gibbons H, Win J \& Lipski J (1999). Effects of cyanide and hypoxia on membrane currents in neurones acutely dissociated from the rostral ventrolateral medulla of the rat. Brain Res 830, 246-257.

Kong J \& Xu Z (1998). Massive mitochondrial degeneration in motor neurons triggers the onset of amyotrophic lateral sclerosis in mice expressing a mutant SOD1. J Neurosci 18, 3241-3250.

Koyama S, Jin YH \& Akaike N (1999). ATP-sensitive and $\mathrm{Ca}^{2+}$-activated $\mathrm{K}^{+}$channel activities in the rat locus coeruleus neurons during metabolic inhibition. Brain Res 828, 189-192.

Kruman, II, Pedersen WA, Springer JE \& Mattson MP (1999). ALS-linked Cu/Zn-SOD mutation increases vulnerability of motor neurons to excitotoxicity by a mechanism involving increased oxidative stress and perturbed calcium homeostasis. Exp Neurol 160, 28-39.

Kulik A, Brockhaus J, Pedarzani P \& Ballanyi K (2002). Chemical anoxia activates ATP-sensitive and blocks $\mathrm{Ca}(2+)$-dependent $\mathrm{K}(+)$ channels in rat dorsal vagal neurons in situ. Neuroscience 110, 541-554. 
Kuo J, Fu R, Siddique T \& Heckman CJ (2002). Persistent inward currents from SOD1 transgenic mouse spinal cultures. Soc. Neurosci. Abstr. 789.7.

Kuo JJ, Schonewille M, Siddique T, Schults AN, Fu R, Bar PR, Anelli R, Heckman CJ \& Kroese $A B$ (2003). Hyperexcitability of cultured spinal motoneurons from presymptomatic ALS mice. J Neurophysiol in print.

Ladewig T \& Keller BU (2000). Simultaneous patch-clamp recording and calcium imaging in a rhythmically active neuronal network in the brainstem slice preparation from mouse. Pflügers Arch 440, 322-332.

Ladewig T, Kloppenburg P, Lalley PM, Zipfel WR, Webb WW \& Keller BU (2003). Spatial profiles of store-dependent calcium release in motoneurones of the nucleus hypoglossus from newborn mouse. J Physiol 547.3, 775-787.

Lawrie AM, Rizzuto R, Pozzan T \& Simpson AW (1996). A role for calcium influx in the regulation of mitochondrial calcium in endothelial cells. J Biol Chem 271, 10753-10759.

Lips MB \& Keller BU (1998). Endogenous calcium buffering in motoneurones of the nucleus hypoglossus from mouse. J Physiol 511.1, 105-117.

Lips MB \& Keller BU (1999). Activity-related calcium dynamics in motoneurons of the nucleus hypoglossus from mouse. J Neurophysiol 82, 2936-2946.

Liu Q \& Wong-Riley MT (2003). Postnatal changes in cytochrome oxidase expressions in brain stem nuclei of rats: implications for sensitive periods. $J$ Appl Physiol 95, 2285-2291.

Lopez-Barneo J, Pardal R \& Ortega-Saenz P (2001). Cellular mechanism of oxygen sensing. Annu Rev Physiol 63, 259-287.

MacGregor DG, Avshalumov MV \& Rice ME (2003). Brain edema induced by in vitro ischemia: causal factors and neuroprotection. $J$ Neurochem 85, 1402-1411.

Menzies FM, Cookson MR, Taylor RW, Turnbull DM, Chrzanowska-Lightowlers ZM, Dong L, Figlewicz DA \& Shaw PJ (2002a). Mitochondrial dysfunction in a cell culture model of familial amyotrophic lateral sclerosis. Brain 125, 1522-1533.

Menzies FM, Ince PG \& Shaw PJ (2002b). Mitochondrial involvement in amyotrophic lateral sclerosis. Neurochem Int 40, 543-551.

Mironov SL (1995). Plasmalemmal and intracellular $\mathrm{Ca}^{2+}$ pumps as main determinants of slow $\mathrm{Ca}^{2+}$ buffering in rat hippocampal neurones. Neuropharmacology 34, 1123-1132.

Müller M, Brockhaus J \& Ballanyi K (2002). ATP-independent anoxic activation of ATP-sensitive $\mathrm{K}^{+}$channels in dorsal vagal neurons of juvenile mice in situ. Neuroscience 109, 313-328. 
Neher E (1995). The use of fura-2 for estimating $\mathrm{Ca}^{2+}$ buffers and $\mathrm{Ca}^{2+}$ fluxes. Neuropharmacology 34, 1423-1442.

Neher E \& Augustine GJ (1992). Calcium gradients and buffers in bovine chromaffin cells. J Physiol 450, 273-301.

Nicholls DG \& Budd SL (2000). Mitochondria and neuronal survival. Physiol Rev 80, 315-360.

Nowicky AV \& Duchen MR (1998). Changes in $\left[\mathrm{Ca}^{2+}\right]$ i and membrane currents during impaired mitochondrial metabolism in dissociated rat hippocampal neurons. $J$ Physiol 507, 131-145.

Palecek J, Lips MB \& Keller BU (1999). Calcium dynamics and buffering in motoneurones of the mouse spinal cord. J Physiol 520.2, 485-502.

Powers RK \& Binder MD (2003). Persistent sodium and calcium currents in rat hypoglossal motoneurons. J Neurophysiol 89, 615-624.

Ramirez JM, Quellmalz UJ \& Wilken B (1997). Developmental changes in the hypoxic response of the hypoglossus respiratory motor output in vitro. $J$ Neurophysiol 78, 383-392.

Reaume AG, Elliott JL, Hoffman EK, Kowall NW, Ferrante RJ, Siwek DF, Wilcox HM, Flood DG, Beal MF, Brown RH, Jr., Scott RW \& Snider WD (1996). Motor neurons in $\mathrm{Cu} / \mathrm{Zn}$ superoxide dismutase-deficient mice develop normally but exhibit enhanced cell death after axonal injury. Nat Genet 13, 43-47.

Rizzuto R, Bernardi P \& Pozzan T (2000). Mitochondria as all-round players of the calcium game. J Physiol $529 \mathrm{Pt}$ 1, 37-47.

Rizzuto R, Pinton P, Brini M, Chiesa A, Filippin L \& Pozzan T (1999). Mitochondria as biosensors of calcium microdomains. Cell Calcium 26, 193-199.

Rosen DR, Siddique T, Patterson D, Figlewicz DA, Sapp P, Hentati A, Donaldson D, Goto J, O'Regan JP, Deng HX, Rahmani Z, Krizus A, McKenna-Yasek D, Cayabyab A, Gaston SM, Berger R, Tanzi RE, Halperin JJ, Herzfeld B, Van den Bergh R, Hung WY, Bird T, Deng G, Mulder DW, Smyth C, Laing NG, Soriano E, Pericak-Vancell MA, Haines J, Rouleau GA, Gusella JS, Horvitz HR \& Brown Jr RH (1993). Mutations in Cu/Zn superoxide dismutase gene are associated with familial amyotrophic lateral sclerosis. Nature 364, 362.

Rothstein JD, Martin LJ \& Kuncl RW (1992). Decreased glutamate transport by the brain and spinal cord in amyotrophic lateral sclerosis. $N$ Engl $J$ Med 326, 1464-1468.

Rowland LP \& Shneider NA (2001). Amyotrophic lateral sclerosis. N Engl J Med 344, 1688-1700. 
Roy J, Minotti S, Dong L, Figlewicz DA \& Durham HD (1998). Glutamate potentiates the toxicity of mutant $\mathrm{Cu} / \mathrm{Zn}$-superoxide dismutase in motor neurons by postsynaptic calcium-dependent mechanisms. J Neurosci 18, 9673-9684.

Sasaki S \& Iwata M (1996). Ultrastructural study of synapses in the anterior horn neurons of patients with amyotrophic lateral sclerosis. Neurosci Lett 204, 5356.

Schuchmann S, Lückermann M, Kulik A, Heinemann U \& Ballanyi K (2000). Ca(2+)and metabolism-related changes of mitochondrial potential in voltage-clamped CA1 pyramidal neurons in situ. J Neurophysiol 83, 1710-1721.

Shaw PJ \& Eggett CJ (2000). Molecular factors underlying selective vulnerability of motor neurons to neurodegeneration in amyotrophic lateral sclerosis. $J$ Neurol 247 Suppl 1, 117-27.

Siklos L, Engelhardt J, Harati Y, Smith RG, Joo F \& Appel SH (1996). Ultrastructural evidence for altered calcium in motor nerve terminals in amyotropic lateral sclerosis. Ann Neurol 39, 203-216.

Siklos L, Engelhardt JI, Alexianu ME, Gurney ME, Siddique T \& Appel SH (1998). Intracellular calcium parallels motoneuron degeneration in SOD-1 mutant mice. J Neuropathol Exp Neurol 57, 571-587.

Smith JC, Ellenberger HH, Ballanyi K, Richter DW \& Feldman JL (1991). PreBotzinger complex: a brainstem region that may generate respiratory rhythm in mammals. Science 254, 726-729.

Swerdlow RH, Parks JK, Cassarino DS, Trimmer PA, Miller SW, Maguire DJ, Sheehan JP, Maguire RS, Pattee G, Juel VC, Phillips LH, Tuttle JB, Bennett JP, Jr., Davis RE \& Parker WD, Jr. (1998). Mitochondria in sporadic amyotrophic lateral sclerosis. Exp Neurol 153, 135-142.

Taylor JR (1982). An Introduction to Error Analysis. University Science Books, Oxford University Press.

Van Den Bosch L, Schwaller B, Vleminckx V, Meijers B, Stork S, Ruehlicke T, Van Houtte E, Klaassen H, Celio MR, Missiaen L, Robberecht W \& Berchtold MW (2002). Protective effect of parvalbumin on excitotoxic motor neuron death. Exp Neurol 174, 150-161.

Vandenberghe W, Ihle EC, Patneau DK, Robberecht W \& Brorson JR (2000). AMPA receptor current density, not desensitization, predicts selective motoneuron vulnerability. J Neurosci 20, 7158-7166.

Vanselow BK \& Keller BU (2000). Calcium dynamics and buffering in oculomotor neurones from mouse that are particularly resistant during amyotrophic lateral sclerosis (ALS)-related motoneurone disease. J Physiol 525.2, 433-445. 
Vergun O, Sobolevsky Al, Yelshansky MV, Keelan J, Khodorov BI \& Duchen MR (2001). Exploration of the role of reactive oxygen species in glutamate neurotoxicity in rat hippocampal neurones in culture. J Physiol 531, 147-163.

Voet D \& Voet JG (1995). Biochemistry. John Wiley \& Sons, Inc.

Werth JL \& Thayer SA (1994). Mitochondria buffer physiological calcium loads in cultured rat dorsal root ganglion neurons. J Neurosci 14, 348-356.

Wiedemann FR, Manfredi G, Mawrin C, Beal MF \& Schon EA (2002). Mitochondrial DNA and respiratory chain function in spinal cords of ALS patients. $J$ Neurochem 80, 616-625.

Wong-Riley MT (1989). Cytochrome oxidase: an endogenous metabolic marker for neuronal activity. Trends Neurosci 12, 94-101.

Xu T, Naraghi M, Kang H \& Neher E (1997). Kinetic studies of $\mathrm{Ca}^{2+}$ binding and $\mathrm{Ca}^{2+}$ clearance in the cytosol of adrenal chromaffin cells. Biophys $J 73,532-545$. 


\section{Curriculum Vitae}

\section{Friederike Bergmann}

born: 28 January 1974 in Wuppertal, Germany

\section{Education}

1993 High school degree (Abitur), Wilhelm-Dörpfeld-Gymnasium Wuppertal

1993-2000 Medical school, Albert-Ludwigs-Universität Freiburg, Germany, and Leopold-Franzens-Universität Innsbruck, Austria

2000 Dr. med., University of Freiburg, Germany

\section{Clinical experience}

2000-2001 Internship, Dept. of Clinical Neurophysiology, University of Göttingen, Germany

Research activity

1996-1998 MD student, Dept. of Physiology, University of Innsbruck, Austria (supervisor: Prof. Markus Paulmichl)

2000-2003 PhD student (International Neuroscience Study Program), Dept. of Neurophysiology, University of Göttingen, Germany (supervisor: Prof. Bernhard U. Keller),

Member of the European Graduiertenkolleg "Neuroplasticity: from molecules to systems"

11/2001 Visiting scientist at the Dept. of Physiology, University College London, U.K.

08/2003 Scientific fellow at the Dept. of Applied \& Engineering Physics, Cornell University, Ithaca, NY, U.S.A. (head: Prof. Watt W. Webb)

Teaching activity

2001-2003 Neurophysiology courses for MD students in Göttingen

(Praktikum, Seminar, Einführung)

2001-2002 Membrane- and Neurophysiology courses for Master students of the International Neuroscience Study program in Göttingen 


\section{Publications}

\section{Articles}

Vanselow BK, Fuchs J, Bergmann F, Frermann D, Keller, BU. A quantitative model of calcium profiles in motoneurons that are selectively vulnerable in the mutant SOD1 G93A mouse model of human amyotrophic lateral sclerosis (ALS). Submitted.

Bergmann F, Keller BU. Quantitative evaluation of mitochondrial $\mathrm{Ca}^{2+}$ clearance in hypoglossal motoneurons from mouse. Submitted.

Bergmann F, Keller BU. Impact of mitochondrial inhibition on excitability and cytosolic $\mathrm{Ca}^{2+}$ levels in brain stem motoneurons from mouse. $\mathrm{J}$ Physiol; in press.

*Stringaris AK, Geisenhainer J, *Bergmann F, Balshüsemann C, Lee U, Zysk G, Mitchell TJ, Keller BU, Kuhnt U, Gerber J, Bähr M, Michel U, Nau R. Neurotoxicity of Pneumolysin, a major Pneumococcal Virulence Factor, Involves Calcium Influx and Depends on Activation of p38 Mitogen Activated Protein Kinase. Neurobiol Dis 2002; 11(3):355-68. ( ${ }^{*}$ e.c.)

Bergmann F, Bleich S, Wischer S, Paulus W. Seizure and Cardiac arrest during Bupropion SR treatment. J Clin Psychopharmacol 2002; 22(6):630-1.

Bergmann F, Krone B, Bleich S, Prange H, Paulus W. Encephalitis due to a Hantavirus Infection. J Infect 2002; 45(1):58-9.

Scandella E, Nagl UO, Oehl B, Bergmann F, Gschwentner M, Furst J, Schmarda A, Ritter M, Waldegger S, Lang F, Deetjen P, Paulmichl M. The promoter for constitutive expression of the human ICIn gene CLNS1A. J Biol Chem 2000; 275(21):15613-20.

Nagl UO, Erdel M, Bergmann F, Oehl B, Scandella E, Musante L, Galietta LJ, Gschwentner M, Furst J, Schmarda A, Hofer S, Uttermann G, Deetjen P, Paulmichl M. Characterization of the human gene coding for the swelling-dependent chloride channel ICIn at position 11q13.5-14.1 (CLNS1A) and further characterization of the chromosome 6 (CLNS1B) localization. Gene 1998; 209(1-2):59-63.

\section{Book chapter}

Bergmann F, Keller BU (2003). Glutamate, calcium and neurodegenerative disease: the impact of cytosolic buffer systems and their potential role for neuroprotective strategies. In: Brain Damage and Repair. T. Herdegen (Ed.). Kluwer Press. In press. 